\title{
Renewal Theory for a System with Internal States
}

\author{
M. Niemann ${ }^{1,2}$, E. Barkai ${ }^{3}$, H. Kantz ${ }^{2}$ \\ ${ }^{1}$ Institut für Physik, Carl von Ossietzky Universität Oldenburg, 26111 Oldenburg, Germany \\ ${ }^{2}$ Max-Planck-Institut für Physik komplexer Systeme, Nöthnitzer Straße 38, 01187 Dresden, Germany \\ ${ }^{3}$ Department of Physics, Bar Ilan University, Ramat-Gan 52900, Israel
}

\begin{abstract}
We investigate a stochastic signal described by a renewal process for a system with $N$ states. Each state has an associated joint distribution for the signal's intensity and its holding time. We calculate multi-point distributions, correlation functions, and the powerspectrum of the signal. Focusing on fat tailed power-law distributed sojourn times in the states of the system, we investigate $1 / f$ noise in this widely applicable model. When the mean waiting time is infinite, the averaged sample spectrum depends both on the age of the process, i.e. the time elapsing from start of the process and the start of observation, and on the total time of observation. Fluctuations of the periodogram estimator of the power-spectrum are investigated for aged systems and are found to be determined by the distribution of the number of renewals in the observation time window. These reduce to the Mittag-Leffler distribution when the start of observation is also the start of the process. When the average waiting time is finite we find a time independent Wienerian spectrum computed from the stationary correlation function of the signal.
\end{abstract}

Keywords and phrases: Renewal theory, multi-point distributions, ageing, $1 / f$-noise, weak ergodicity breaking, Wiener Khinchin theorem, Mittag-Leffler-distribution

Mathematics Subject Classification: 60K05, 62M15

\section{Introduction}

Renewal theory is the branch of probability theory that generalizes Poisson processes for arbitrary holding times [16]. A far less general class of processes, called fractal renewal processes [27], was extensively studied by mathematicians $[17,28]$, and the statistical physical community $[18,21,42,49]$. An early application is the work of Berger and Mandelbrot on communication networks [9]. Here fat tailed probability density functions of the sojourn times are described by $\psi(t) \sim t^{-(1+\alpha)}$. When $0<\alpha<1$ the average waiting/holding/sojourn time is infinite. This leads to anomalous diffusion [36], aging [4,38], $1 / f$ noise [44] and weak ergodicity breaking $[8,12,30]$, and hence these type of processes have attracted large attention. A few examples are renewal processes describing blinking quantum dots [23, 47], diffusion of tracer particles in disordered systems [13] like the cell environment [5,35], chaotic systems [2, 25], diffusion of cold atoms in optical lattices [3], line shapes in complex systems [45], brain activity and music [10] experiments on Kardar Parisi Zhang interfaces [48], and social networks [37]. Mathematically the limit theorems of continuous time random walks and subordination have attracted considerable attention

\footnotetext{
${ }^{*}$ Corresponding author. E-mail: Eli.Barkai@biu.ac.il
} 
[32-34]. Indeed maybe the best well known example of a fractal renewal process is the continuous time random walk model where the particle's position $x(t)$ is described by random jump events followed by power law distributed trapping times in which the particle is immobile, leading to sub-diffusion [36]. In mathematical modeling a state function is changing its value on dots on the time axis, when the process is renewed. The state function can model the position of a particle in a medium with deep traps or the intensity of light of a blinking quantum dot, which switches between a dark and bright state. The number of states can vary significantly among different models. Further the value of the state function, can be random itself. For example the intensity of the bright state of a blinking dot fluctuates or a trapped particle can jiggle around its averaged position before making large jumps which defines a renewal event [22]. While certain aspects of this general problem where treated previously, here we provide a detailed investigation of renewal theory for a system with internal states (see below). Our goal is to expose the general mathematical aspects of this process, but we also investigate in detail one application which is the power spectrum of a fractal time renewal processes. Here the internal fluctuations of the state function clearly contribute to the noise level, which can be detected by power-spectrum analysis.

More specifically, as shown both theoretically and experimentally the $1 / f$ power spectrum of a blinking quantum dot ages $[31,44]$. Namely the power spectrum estimated with the periodogram method depends on the measurement time. A non-Wienerian spectral theory was recently introduced to describe a general class of such non-stationary processes $[15,26]$. The power spectrum of blinking quantum dots was the topic of our recent publication [40] were we focused on two aspects of the problem. The first was the solution of the well known low frequency cut-off paradox [29,43], namely the non integrability of $1 / f^{\beta}$ power spectrum when $\beta>1$ which paradoxically indicated non-normalized power spectrum and infinite energy of the process. The second aspect of our work was to characterize the fluctuations of the spectrum and its aging. One of the goals of this paper is to provide an extensive analysis of the power spectrum for a general renewal setting. Thus we go beyond the two state models we considered previously (the latter is reasonable for quantum dots but not for other systems). The diverging sojourn times in on and off states, implies that the sample spectrum is random, and we here quantify this effect in detail. We also consider aged processes, namely the case where the start of observation does not coincide with the start of the process, showing how in scale free dynamics the power spectrum crucially depends on the age.

The navigation map of this paper is as follows. We first introduce the model and definitions followed by the investigation of the multi-point distribution function of the process (Sec. II and III). Aged systems, correlation functions for both aged and non-aged processes follow (Sec. IV-VI). Stationary behavior, when the average sojourn time is finite, is discussed in Sec. VII. The remaining sections (VII-X) deal with the Wienerian $\alpha>1$ and non-Wienerian $\alpha<1$ power spectrums, both for the aged and non-aged processes, quantifying the fluctuations of the spectrum in detail. Sec. $\mathrm{X}$ is a summary.

\section{The Model and Notations}

\subsection{The Model}

In this section we describe the stochastic model used in this paper. We look at a stochastic signal: at time $t$ the signal has the intensity $X(t)$. Our process starts at the time $T=0$. For convenience we define $X(t)=0$ for $t<0$.

The process itself consists of two parts which are interweaved. One part describes directly the observed intensity, the other part describes the internal state of the system which cannot be observed directly. First, we will describe the internal state space. In this paper we will only consider the case of finitely many internal states. The number of states is denoted by $N$. The dynamics on the internal states is described by a Markov chain. Formally, it is given by a (left) stochastic matrix $M$ of dimension $N \times N$. For convenience, we adopt the Bra-Ket notation: the bras $\langle\cdots|$ denote row vectors and the kets $|\cdots\rangle$ denote column vectors. The term $\langle i|$ denotes the row vector having a 1 at the $i$ th position and 0 elsewhere. Correspondingly, $|j\rangle$ is the column vector with 1 at the $j$ th position and 0 elsewhere. Then, the transition probability from state $i$ to state $j$ is given by $\langle i|M| j\rangle$. We assume that $M$ is ergodic, i.e., we have a 


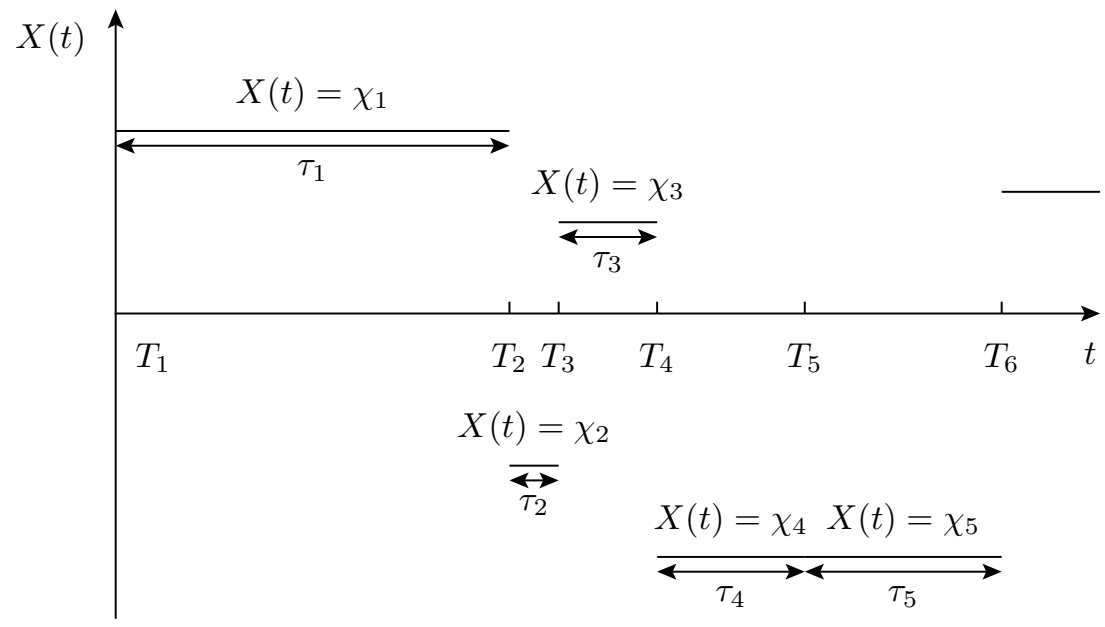

FiguRE 1. Exemplary illustration of a process

unique equilibrium distribution $\left\langle\mathrm{eq}_{\mathrm{M}}\right|$ with $\left\langle\mathrm{eq}_{\mathrm{M}}\right| M=\left\langle\mathrm{eq}_{\mathrm{M}}\right|$. The corresponding right eigenvector $|\Sigma\rangle$ consists of 1s at every position $|\Sigma\rangle=\sum_{i=1}^{N}|i\rangle$ and we have $M|\Sigma\rangle=|\Sigma\rangle$. We still need to fix the initial conditions. For this, we provide the probability distribution of the states at the start of the process which we denote $\langle$ init|. The probability for the first epoch to be in state $i$ is $\langle$ init $\mid i\rangle$.

The internal state determines the current behavior of the process. Each internal state $m$ has an associated distribution for intensity and waiting time which is denoted by $\phi_{m}(x, t)$. The process is divided into epochs: in each epoch $j$ the system is in one internal state $m_{j}\left(1 \leq m_{j} \leq N\right)$. An intensity $\chi_{j}$ and a waiting time $\tau_{j}$ is drawn according to the probability density $\phi_{m_{j}}(x, t)$. They are drawn independently from the intensity and waiting times in the other epochs. If we are at the beginning of the $j$ th epoch at a time $T_{j}$, the process $X(t)$ takes the value $\chi_{j}$ for a time interval starting at $T_{j}$ of length $\tau_{j}$. In other words $X(t)=\chi_{j}$ for $T_{j} \leq t<T_{j}+\tau_{j}$. At time $T_{j+1}=T_{j}+\tau_{j}$ starts the next epoch $j+1$. For this epoch the internal state $m_{j}$ is advanced to a new state $m_{j+1}$ according to the Markov chain $M$. This means, the state $m_{j+1}$ is drawn according to the probability distribution $\left\langle m_{j}\right| M$. The process is illustrated in Figure 1.

We have defined the process such that the first epoch starts at $T_{1}=0$. The initial condition is the distribution of the initial states 〈init| for the first epoch. The ensemble average with respect to this distribution is denoted by $\langle\cdot\rangle_{\langle\text {init }}$. Sometimes the initial distribution is clear from context (or does not play any role, as for some asymptotic properties), we will then drop the index. We additionally introduce the stochastic process $N(t)$ which denotes the internal state the process is in at time $t$.

In the following, we introduce some notation which is useful for the formal treatment of this process. The Laplace transform in time of the distributions $\phi_{j}(x, t)$ is

$$
\hat{\phi}_{m}(x, \lambda)=\int_{0}^{\infty} \mathrm{d} t \exp (-\lambda t) \phi_{m}(x, t) .
$$

The formula is valid for $\lambda \in \mathbb{C}$ with $\Re \lambda \geq 0$. The connection to the Fourier transform can be obtained via

$$
\int_{-\infty}^{\infty} \mathrm{d} t \exp (i \omega t) \phi_{m}(x, t)=\hat{\phi}_{m}(x,-i \omega)
$$

where we set $\phi_{m}(x, t)=0$ for $t<0$ as we have no negative waiting times. We group these distributions in diagonal matrices

$$
\Phi(x, t)=\operatorname{diag}\left(\phi_{1}(x, t), \ldots, \phi_{N}(x, t)\right), \quad \hat{\Phi}(x, \lambda)=\operatorname{diag}\left(\hat{\phi}_{1}(x, \lambda), \ldots, \hat{\phi}_{N}(x, \lambda)\right) .
$$


We assume that all moments in $x$ exists $(n=0,1,2, \ldots)$

$$
\int_{0}^{\infty} \mathrm{d} t \int_{-\infty}^{\infty} \mathrm{d} x x^{n} \phi_{j}(x, t)<\infty
$$

and we define

$$
\begin{aligned}
\Psi_{n}(t) & =\int_{-\infty}^{\infty} \mathrm{d} x x^{n} \Phi(x, t) \\
\hat{\Psi}_{n}(\lambda) & =\int_{-\infty}^{\infty} \mathrm{d} x x^{n} \hat{\Phi}(x, \lambda)=\int_{0}^{\infty} \mathrm{d} t \exp (-\lambda t) \Psi_{n}(t) .
\end{aligned}
$$

Because of Eq. (2.4), the entries of $\hat{\Psi}_{n}(0)$ are finite. The elements of $\Psi_{0}(t)$ are the marginal distributions for the waiting times. Averaging over the internal states with the equilibrium distribution leads to the definitions

$$
\begin{aligned}
\bar{\phi}(x, t) & =\left\langle\mathrm{eq}_{\mathrm{M}}|\Phi(x, t)| \Sigma\right\rangle \\
\bar{\psi}_{n}(t) & =\left\langle\mathrm{eq}_{\mathrm{M}}\left|\Psi_{n}(t)\right| \Sigma\right\rangle \\
\overline{\bar{\psi}}_{n}(\lambda) & =\left\langle\mathrm{eq}_{\mathrm{M}}\left|\hat{\Psi}_{n}(\lambda)\right| \Sigma\right\rangle .
\end{aligned}
$$

\subsection{Asymptotic Behavior}

The behavior of the long waiting times is described by the small $\lambda$ behavior of $\hat{\Psi}_{0}(\lambda)$. We assume that we can expand $\left(\hat{\Psi}_{0}(0)=\mathbf{I}\right)$

$$
\hat{\Psi}_{0}(\lambda)=\mathbf{I}-\lambda^{\alpha} K_{0}+\mathrm{o}\left(\lambda^{\alpha}\right)
$$

with $0<\alpha \leq 1$ and $\mathrm{o}\left(\lambda^{\alpha}\right)$ being the Landau symbol. $K_{0}$ is a diagonal matrix with non-negative entries. The case $\alpha=1$ appears when all waiting times have a finite mean. In the case of $0<\alpha<1$ at least one of the waiting times has infinite mean and the probability density for large waiting times behaves asymptotically as $1 / t^{1+\alpha}$. It is not necessary that all diagonal elements of $K_{0}$ are larger than zero, i.e., that all waiting time distribution individually would result in the same $\alpha$. We only need

$$
\bar{\tau}^{\alpha}=\left\langle\mathrm{eq}_{\mathrm{M}}\left|K_{0}\right| \Sigma\right\rangle>0 .
$$

Therefore the averaged waiting time $\hat{\bar{\psi}}_{0}(\lambda)$ has the expansion

$$
\hat{\bar{\psi}}_{0}(\lambda)=\left\langle\mathrm{eq}_{\mathrm{M}}\left|\hat{\Psi}_{0}(\lambda)\right| \Sigma\right\rangle=1-(\bar{\tau} \lambda)^{\alpha}+\mathrm{o}\left(\lambda^{\alpha}\right) .
$$

Some time, we additionally assume, that we also have an expansion for the moments

$$
\hat{\Psi}_{n}(\lambda)=\hat{\Psi}_{n}(0)-\lambda^{\alpha} K_{n}+\mathrm{o}\left(\lambda^{\alpha}\right)
$$

where - in contrast to the $n=0$ case - we also allow $K_{n}=0$. This assumption is not unreasonable, we will later show that the asymptotic moments of $X(t)$ are (see Eq. (C.5))

$$
\begin{aligned}
\mu_{n}^{\text {asym }} & =\lim _{t \rightarrow \infty}\left\langle X^{n}(t)\right\rangle_{\langle\text {init }|} \\
& =\frac{\left\langle\mathrm{eq}_{\mathrm{M}}\left|K_{n}\right| \Sigma\right\rangle}{\left\langle\mathrm{eq}_{\mathrm{M}}\left|K_{0}\right| \Sigma\right\rangle} .
\end{aligned}
$$

Physically, this expansion is reasonable as it simply requires that the moments $\left\langle X^{n}(t)\right\rangle$ stay finite for large $t$. 
A helpful way to interpret some results, is the use of the distribution $\bar{\phi}^{\text {lt }}(x)$ which describe the long-time behavior of $\bar{\phi}(x, t)$. We condition the distribution of $x$ on $t>T$ and then let $T \rightarrow \infty$ :

$$
\bar{\phi}^{\mathrm{lt}}(x)=\lim _{T \rightarrow \infty} \frac{\int_{T}^{\infty} \mathrm{d} t \bar{\phi}(x, t)}{\int_{T}^{\infty} \mathrm{d} t \int_{-\infty}^{\infty} \mathrm{d} x \bar{\phi}(x, t)} .
$$

Its moments are

$$
\bar{\psi}_{n}^{\mathrm{lt}}=\int_{-\infty}^{\infty} \mathrm{d} x x^{n} \bar{\phi}^{\mathrm{lt}}(x)
$$

and its variance

$$
\sigma_{\mathrm{lt}}^{2}=\bar{\psi}_{2}^{\mathrm{tt}}-\left(\bar{\psi}_{1}^{\mathrm{lt}}\right)^{2}
$$

To illustrate our findings, we will employ three different example models:

1. The single state model: it is a simple model without internal states. Formally, we will have $N=1$ internal state and the Markov matrix is $M=(1)$. Therefore, we can drop the matrix notation completely. The probability distribution for the intensity/waiting time is denoted by $\phi^{\text {single }}(x, t)$ with the self-explanatory notations $\psi_{i}^{\text {single }}(t)$ and $\hat{\psi}_{i}^{\text {single }}(\lambda)$. We assume the following expansion of the Laplace transform of the waiting time

$$
\hat{\psi}_{0}^{\text {single }}(\lambda)=1-\bar{\tau}_{\text {single }}^{\alpha} \lambda^{\alpha}+\mathrm{o}\left(\lambda^{\alpha}\right) .
$$

Remark: any process with a decoupling Markov chain, i.e., the next internal state is drawn from the equilibrium distribution independently of the current state (formally $M=|\Sigma\rangle\left\langle\mathrm{eq}_{\mathrm{M}}\right|$ ) with initial distribution being the equilibrium one $\langle$ init $|=\left\langle\mathrm{eq}_{\mathrm{M}}\right|$, is equivalent to this process by letting $\phi^{\text {single }}(x, t)=\bar{\phi}(x, t)$.

2. The alternating model: it has two states named + and - between which it switches alternatingly. The Markov matrix for this model is

$$
M^{\text {alt }}=\left(\begin{array}{ll}
0 & 1 \\
1 & 0
\end{array}\right)
$$

which has the equilibrium distribution

$$
\left\langle\mathrm{eq}_{\mathrm{M}}\right|=\left(\frac{1}{2} \frac{1}{2}\right)
$$

We assume that the intensity of the process is $I_{+}$(resp. $I_{-}$) in the + state (resp. - state), i.e.,

$$
X(t)=I_{+} \text {or } I_{-} .
$$

The waiting time distribution is the same for both states and is denoted $\psi^{\text {alt }}(t)$. Taking the first state to be the + state gives the distribution matrix

$$
\Phi^{\text {alt }}(x, t)=\left(\begin{array}{cc}
\delta\left(x-I_{+}\right) \psi^{\text {alt }}(t) & 0 \\
0 & \delta\left(x-I_{-}\right) \psi^{\text {alt }}(t)
\end{array}\right) .
$$

We assume that the Laplace transforms of the waiting time distribution has the following small $\lambda$ behavior

$$
\hat{\psi}^{\text {alt }}(\lambda)=1-\bar{\tau}^{\alpha} \lambda^{\alpha}+o\left(\lambda^{\alpha}\right)
$$

with $0<\alpha<1$. As a concrete example: they could be a one-sided Lévy stable distribution $\left(\hat{\psi}^{\text {alt }}(\lambda)=\right.$ $\left.\exp \left(-\bar{\tau}^{\alpha} \lambda^{\alpha}\right)\right)$. Important is that they have the same long time behavior, i.e., lead to the same $\alpha$.

The Laplace transform of $\Phi^{\text {alt }}(x, t)$ is then

$$
\hat{\Phi}^{\mathrm{alt}}(x, \lambda)=\left(\begin{array}{cc}
\delta\left(x-I_{+}\right) \hat{\psi}^{\mathrm{alt}}(\lambda) & 0 \\
0 & \delta\left(x-I_{-}\right) \hat{\psi}^{\mathrm{alt}}(\lambda)
\end{array}\right) .
$$


The moment matrices are then

$$
\begin{aligned}
\hat{\Psi}_{n}^{\mathrm{alt}}(\lambda) & =\left(\begin{array}{cc}
I_{+}^{n} \hat{\psi}^{\mathrm{alt}}(\lambda) & 0 \\
0 & I_{-}^{n} \hat{\psi}^{\mathrm{alt}}(\lambda)
\end{array}\right) \\
& =\left(\begin{array}{cc}
I_{+}^{n} & 0 \\
0 & I_{-}^{n}
\end{array}\right)-\lambda^{\alpha}\left(\begin{array}{cc}
\bar{\tau}^{\alpha} I_{+}^{n} & 0 \\
0 & \bar{\tau}^{\alpha} I_{-}^{n}
\end{array}\right)+\mathrm{o}\left(\lambda^{\alpha}\right) .
\end{aligned}
$$

Therefore, the expansion matrices are

$$
K_{n}=\left(\begin{array}{cc}
\bar{\tau}^{\alpha} I_{+}^{n} & 0 \\
0 & \bar{\tau}^{\alpha} I_{-}^{n}
\end{array}\right)
$$

The long time distribution is

$$
\bar{\phi}^{\mathrm{lt}}(x)=\frac{1}{2}\left(\delta\left(x-I_{+}\right)+\delta\left(x-I_{-}\right)\right)
$$

with variance

$$
\sigma_{\mathrm{lt}}^{2}=\left(\frac{I_{+}-I_{-}}{2}\right)^{2}
$$

One recurring term is $\left[\mathbf{I}-\hat{\Psi}_{0}(\lambda) M^{\text {alt }}\right]^{-1}$ which is calculated here

$$
\begin{aligned}
{\left[\mathbf{I}-\hat{\Psi}_{0}(\lambda) M^{\text {alt }}\right]^{-1} } & =\left(\begin{array}{cc}
1 & -\hat{\psi}^{\text {alt }}(\lambda) \\
-\hat{\psi}^{\text {alt }}(\lambda) & 1
\end{array}\right)^{-1} \\
& =\frac{1}{1-\hat{\psi}^{\text {alt }}(\lambda)^{2}}\left(\begin{array}{cc}
1 & \hat{\psi}^{\text {alt }}(\lambda) \\
\hat{\psi}^{\text {alt }}(\lambda) & 1
\end{array}\right) .
\end{aligned}
$$

3. The burst model: it is very similar to the alternating model, with the difference that while the - state still has an expansion with $0<\alpha<1$, the + state has an expansion with $\tilde{\alpha}(\alpha<\tilde{\alpha}<1)$, i.e.,

$$
\begin{aligned}
& \hat{\psi}_{+}^{\text {burst }}(\lambda)=1-c_{+} \lambda^{\tilde{\alpha}}+o\left(\lambda^{\tilde{\alpha}}\right) \\
& \hat{\psi}_{-}^{\text {burst }}(\lambda)=1-c_{-} \lambda^{\alpha}+o\left(\lambda^{\alpha}\right) .
\end{aligned}
$$

We also allow $\tilde{\alpha}=1$ with the expansion to next order $(0<\tilde{\beta} \leq 1)$

$$
\begin{aligned}
& \hat{\psi}_{+}^{\text {burst }}(\lambda)=1-c_{+} \lambda+c_{+}^{(2)} \lambda^{1+\tilde{\beta}}+\mathrm{o}\left(\lambda^{1+\tilde{\beta}}\right) \\
& \hat{\psi}_{-}^{\text {burst }}(\lambda)=1-c_{-} \lambda^{\alpha}+\mathrm{o}\left(\lambda^{\alpha}\right) .
\end{aligned}
$$

We explicitly allow the case $\tilde{\beta}=1$ which corresponds to an existing second moment of the waiting time

$$
c_{+}^{(2)}=\frac{1}{2} \int_{0}^{\infty} \mathrm{d} t t^{2} \psi_{+}^{\text {burst }}(t)
$$

We will use this model mainly to carve out differences to the alternating model, as here only the value $I_{-}$dominates the long waiting times. We have here

$$
\hat{\Phi}^{\text {burst }}(x, \lambda)=\left(\begin{array}{cc}
\delta\left(x-I_{+}\right) \hat{\psi}_{+}^{\text {burst }}(\lambda) & 0 \\
0 & \delta\left(x-I_{-}\right) \hat{\psi}_{-}^{\text {burst }}(\lambda)
\end{array}\right)
$$

and

$$
\begin{aligned}
& \hat{\Psi}_{n}^{\text {burst }}(\lambda)=\left(\begin{array}{ccc}
I_{+}^{n} \hat{\psi}_{+}^{\text {burst }}(\lambda) & 0 \\
0 & I_{-}^{n} \hat{\psi}_{-}^{\text {burst }}(\lambda)
\end{array}\right) \\
& =\left(\begin{array}{cc}
I_{+}^{n} & 0 \\
0 & I_{-}^{n}
\end{array}\right)+\lambda^{\alpha}\left(\begin{array}{ll}
0 & 0 \\
0 & c_{-} I_{-}^{n}
\end{array}\right)+\mathrm{o}\left(\lambda^{\alpha}\right) .
\end{aligned}
$$


We have here expansion matrices with only one entry differing from zero

$$
K_{n}=\left(\begin{array}{ll}
0 & 0 \\
0 & c_{-} I_{-}^{n}
\end{array}\right)
$$

The long time distribution is

$$
\bar{\phi}^{\mathrm{lt}}(x)=\delta\left(x-I_{-}\right)
$$

with variance

$$
\sigma_{\mathrm{lt}}^{2}=0
$$

\section{Probability Distributions}

\subsection{Main Results}

Assume that at time $t=0$ we are at the beginning of a new epoch where the initial state is drawn from the probability distribution (init|. The value of the process at time $t$ is given by $X(t)$ while its internal state is denoted by $N(t)$. The most basic quantity are the joint probability distributions of having $X\left(t_{j}\right)=x_{j}$ and $N\left(t_{j}\right)=m_{j}$ for all $j \in \mathcal{L}$ where $\mathcal{L}$ is a set of indices:

$$
\left\langle\operatorname{init} \mid P_{\mathcal{L}}\left(\left\{x_{k}\right\},\left\{m_{k}\right\} ;\left\{t_{k}\right\}\right)\right\rangle=\left\langle\prod_{k \in \mathcal{L}} \delta\left(X\left(t_{k}\right)-x_{k}\right) \delta_{N\left(t_{k}\right) m_{k}}\right\rangle_{\langle\text {init }|},
$$

see also $[6,7,39]$. The index $\mathcal{L}$ gives the indices over which the probability distribution is calculated. Therefore, if one wants to calculate the probability distribution for the times $t_{1}, \ldots, t_{n}$ one takes $\mathcal{L}=$ $\{1, \ldots, n\}$

$$
\left\langle\text { init } \mid P_{\{1, \ldots, n\}}\left(\left\{x_{k}\right\},\left\{m_{k}\right\} ;\left\{t_{k}\right\}\right)\right\rangle=\left\langle\prod_{k=1}^{n} \delta\left(X\left(t_{k}\right)-x_{k}\right) \delta_{N\left(t_{k}\right) m_{k}}\right\rangle_{\langle\text {init }|} .
$$

We will additionally use for the $n$-time joint probability distribution with the times $t_{1}, \ldots, t_{n}$ the notation $P_{n}$ :

$$
\left\langle\operatorname{init} \mid P_{n}\left(\left\{x_{1}, \ldots, x_{n}\right\},\left\{m_{1}, \ldots, m_{n}\right\} ;\left\{t_{1}, \ldots, t_{n}\right\}\right)\right\rangle=\left\langle\operatorname{init} \mid P_{\{1, \ldots, n\}}\left(\left\{x_{k}\right\},\left\{m_{k}\right\} ;\left\{t_{k}\right\}\right)\right\rangle .
$$

As $\left\langle\right.$ init $\left.\mid P_{\mathcal{L}}\right\rangle$ is linear in $\langle$ init $|$ we use here the vector notation. For ease of notation we define that

$$
\left|P_{\mathcal{L}}\left(\left\{x_{k}\right\},\left\{m_{k}\right\} ;\left\{t_{k}\right\}\right)\right\rangle=0 \text { if any } t_{k}<0 .
$$

We can determine $\left|P_{\mathcal{L}_{\mathrm{i}}}\left(\left\{x_{k}\right\},\left\{m_{k}\right\} ;\left\{t_{k}\right\}\right)\right\rangle$ by looking at the first epoch (using $\mathcal{L}_{\mathrm{i}}$ for the set of "initial" indices): assuming that we are in state $l=N(0)$, the length of the epoch $\tau$ and its values $\chi$ are distributed according to $\phi_{l}(x, t)$. We have to distinguish two types of indices now: the $k$ for which $t_{k} \geq \tau$ and the $k$ for which $t_{k}<\tau$ (see Figure 2). The indices appearing later are denoted by the set

$$
\mathcal{L}_{\mathrm{f}}=\left\{k \in \mathcal{L}_{\mathrm{i}}: t_{k} \geq \tau\right\}
$$

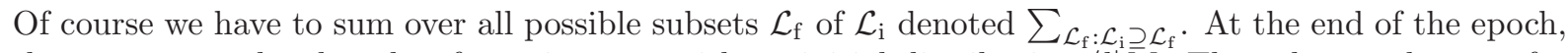
the process can be thought of starting new with an initial distribution $\langle l| \bar{M}$. The values and states for the indices $k \in \mathcal{L}_{\mathrm{i}} \backslash \mathcal{L}_{\mathrm{f}}$ (the difference set) are $\chi$ and $l$ while the values and states for the indices $k \in \mathcal{L}_{\mathrm{f}}$ 
are distributed according to a process starting at $\tau$ with initial distribution $\langle l| M$ :

$\left\langle l \mid P_{\mathcal{L}_{\mathrm{i}}}\left(\left\{x_{k}\right\},\left\{m_{k}\right\} ;\left\{t_{k}\right\}\right)\right\rangle$

$$
\begin{aligned}
& =\sum_{\mathcal{L}_{\mathrm{f}}: \mathcal{L}_{\mathrm{i}} \supseteq \mathcal{L}_{\mathrm{f}}} \int_{0}^{\infty} \mathrm{d} \tau \int_{-\infty}^{\infty} \mathrm{d} \chi\left(\prod_{j \in \mathcal{L}_{\mathrm{i}} \backslash \mathcal{L}_{\mathrm{f}}} \delta_{l m_{j}} \delta\left(\chi-x_{j}\right) \theta\left(\tau-t_{j}\right)\right) \phi_{l}(\chi, \tau)\left\langle l|M| P_{\mathcal{L}_{\mathrm{f}}}\left(\left\{x_{k}\right\},\left\{m_{k}\right\} ;\left\{t_{k}-\tau\right\}\right)\right\rangle \\
& =\left\langle l\left|\sum_{\mathcal{L}_{\mathrm{f}}: \mathcal{L}_{\mathrm{i}} \supseteq \mathcal{L}_{\mathrm{f}}} \int_{0}^{\infty} \mathrm{d} \tau \int_{-\infty}^{\infty} \mathrm{d} \chi\left(\prod_{j \in \mathcal{L}_{\mathrm{i}} \backslash \mathcal{L}_{\mathrm{f}}} \delta_{l m_{j}} \delta\left(\chi-x_{j}\right) \theta\left(\tau-t_{j}\right)\right) \Phi(\chi, \tau) M\right| P_{\mathcal{L}_{\mathrm{f}}}\left(\left\{x_{k}\right\},\left\{m_{k}\right\} ;\left\{t_{k}-\tau\right\}\right)\right\rangle .
\end{aligned}
$$

Here we have used implicitly the definition of $\left|P_{\mathcal{L}_{\mathrm{f}}}\left(\left\{x_{k}\right\},\left\{m_{k}\right\} ;\left\{t_{k}\right\}\right)\right\rangle$ to be $|\Sigma\rangle$ when $\mathcal{L}_{\mathrm{f}}$ is the empty set $\mathcal{L}_{\mathrm{f}}=\{\}:$

$$
\left|P_{\{\}}(\{\},\{\} ;\{\})\right\rangle=|\Sigma\rangle .
$$

We can write (using $\left.\left|P_{\mathcal{L}_{\mathrm{i}}}(\cdots)\right\rangle=\sum_{l}|l\rangle\left\langle l \mid P_{\mathcal{L}_{\mathrm{i}}}(\cdots)\right\rangle\right)$

$$
\begin{aligned}
& \left|P_{\mathcal{L}_{\mathrm{i}}}\left(\left\{x_{k}\right\},\left\{m_{k}\right\} ;\left\{t_{k}\right\}\right)\right\rangle \\
= & \sum_{l}|l\rangle\left\langle l\left|\sum_{\mathcal{L}_{\mathrm{f}}: \mathcal{L}_{\mathrm{i}} \supseteq \mathcal{L}_{\mathrm{f}}} \int_{0}^{\infty} \mathrm{d} \tau \int_{-\infty}^{\infty} \mathrm{d} \chi\left(\prod_{j \in \mathcal{L}_{\mathrm{i}} \backslash \mathcal{L}_{\mathrm{f}}} \delta_{l m_{j}} \delta\left(\chi-x_{j}\right) \theta\left(\tau-t_{j}\right)\right) \Phi(\chi, \tau) M\right| P_{\mathcal{L}_{\mathrm{f}}}\left(\left\{x_{k}\right\},\left\{m_{k}\right\} ;\left\{t_{k}-\tau\right\}\right)\right\rangle \\
= & \sum_{\mathcal{L}_{\mathrm{f}}: \mathcal{L}_{\mathrm{i}} \supseteq \mathcal{L}_{\mathrm{f}}}\left(\prod_{r \in \mathcal{L}_{\mathrm{i}}} \int_{0}^{\infty} \mathrm{d} t_{r}^{\prime}\right) \sum_{l}|l\rangle\langle l| \int_{0}^{\infty} \mathrm{d} \tau \int_{-\infty}^{\infty} \mathrm{d} \chi\left(\prod_{j \in \mathcal{L}_{\mathrm{i}} \backslash \mathcal{L}_{\mathrm{f}}} \delta_{l m_{j}} \delta\left(\chi-x_{j}\right) \theta\left(\tau-t_{j}^{\prime}\right)\right)\left(\prod_{j \in \mathcal{L}_{\mathrm{f}}} \delta\left(\tau-t_{j}^{\prime}\right)\right) \\
& \times \Phi(\chi, \tau) M\left(\prod_{j \in \mathcal{L}_{\mathrm{i}} \backslash \mathcal{L}_{\mathrm{f}}} \delta\left(t_{j}-t_{j}^{\prime}\right)\right)\left|P_{\mathcal{L}_{\mathrm{f}}}\left(\left\{x_{k}\right\},\left\{m_{k}\right\} ;\left\{t_{k}-t_{k}^{\prime}\right\}\right)\right\rangle
\end{aligned}
$$

Therefore, the probability can be written for each $\mathcal{L}_{\mathrm{f}}$ as a convolution in the variables $\left\{t_{k}\right\}$.

The next step is to transfer these results to the Laplace space such we can take advantage of the convolution property of the Laplace transform.

$$
\left|\hat{P}_{\mathcal{L}_{\mathrm{i}}}\left(\left\{x_{k}\right\},\left\{m_{k}\right\} ;\left\{\lambda_{k}\right\}\right)\right\rangle=\left(\prod_{k \in \mathcal{L}_{\mathrm{i}}} \int_{0}^{\infty} \mathrm{d} t_{k} e^{-\lambda_{k} t_{k}}\right)\left|P_{\mathcal{L}_{\mathrm{i}}}\left(\left\{x_{k}\right\},\left\{m_{k}\right\} ;\left\{t_{k}\right\}\right)\right\rangle .
$$

The abbreviation $\Lambda_{\mathcal{J}}=\sum_{j \in \mathcal{J}} \lambda_{j}$ for any set of indices $\mathcal{J}$ will be useful (e.g., $\Lambda_{\{1,2\}}=\lambda_{1}+\lambda_{2}$ and $\Lambda_{\{2,3,5\}}=\lambda_{2}+\lambda_{3}+\lambda_{5}$ ). The Laplace transform of the last line of Eq. (3.8) (written in $\left\{t_{k}\right\}$ ) is

$$
\left(\prod_{k \in \mathcal{L}_{\mathrm{i}}} \int_{0}^{\infty} \mathrm{d} t_{k} e^{-\lambda_{k} t_{k}}\right)\left(\prod_{j \in \mathcal{L}_{\mathrm{i}} \backslash \mathcal{L}_{\mathrm{f}}} \delta\left(t_{j}\right)\right)\left|P_{\mathcal{L}_{\mathrm{f}}}\left(\left\{x_{k}\right\},\left\{m_{k}\right\} ;\left\{t_{k}\right\}\right)\right\rangle=\left|\hat{P}_{\mathcal{L}_{\mathrm{f}}}\left(\left\{x_{k}\right\},\left\{m_{k}\right\} ;\left\{\lambda_{k}\right\}\right)\right\rangle .
$$

The Laplace transform of the second part of the convolution in Eq. (3.8) is expressed by the function

$$
\begin{aligned}
\hat{Q}_{\mathcal{L}_{\mathrm{i}} \mathcal{L}_{\mathrm{f}}}\left(\left\{x_{k}\right\},\left\{m_{k}\right\} ;\left\{\lambda_{k}\right\}\right) \\
=\left(\prod_{k \in \mathcal{L}_{\mathrm{i}}} \int_{0}^{\infty} \mathrm{d} t_{k} e^{-\lambda_{k} t_{k}}\right) \sum_{l}|l\rangle\langle l| \int_{0}^{\infty} \mathrm{d} \tau \int_{-\infty}^{\infty} \mathrm{d} \chi \prod_{j \in \mathcal{L}_{\mathrm{i}} \backslash \mathcal{L}_{\mathrm{f}}} \delta_{l m_{j}} \delta\left(\chi-x_{j}\right) \theta\left(\tau-t_{j}\right) \\
\quad \times \prod_{j \in \mathcal{L}_{\mathrm{f}}} \delta\left(\tau-t_{j}\right) \Phi(\chi, \tau) M .
\end{aligned}
$$




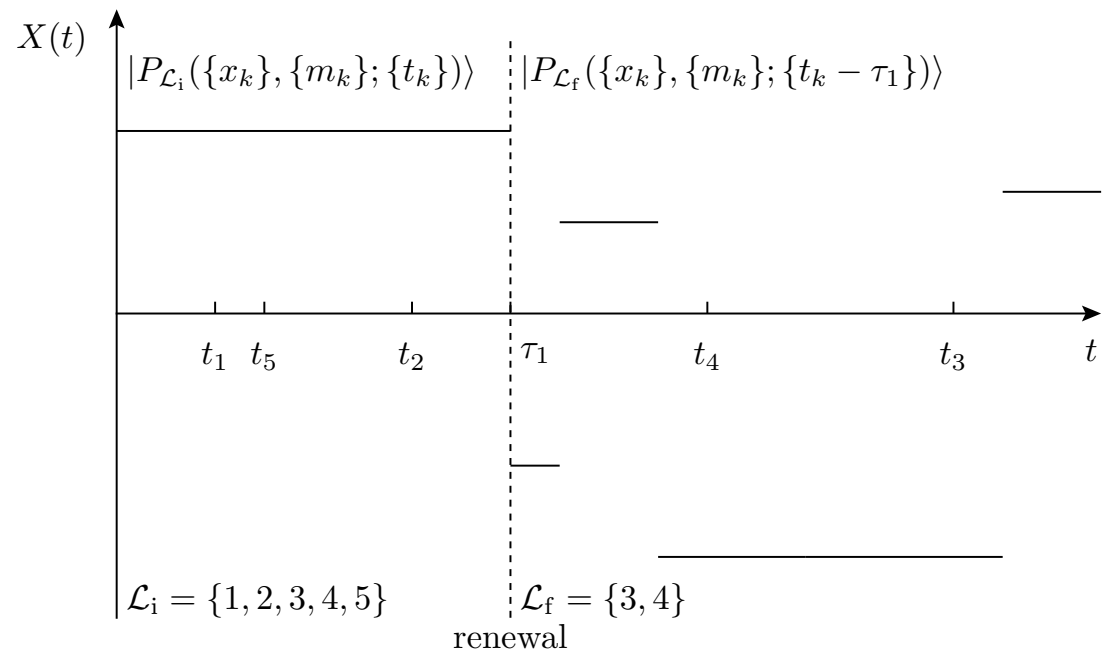

FiguRE 2. Graphical situation for the renewal ansatz: we have the same situation after the first epoch of length $\tau_{1}$, this time point is marked with a dashed line and the word "renewal". At time $t=0$, all observed times are larger (namely $t_{1}, t_{2}, t_{3}, t_{4}, t_{5}$ ), therefore our initial set of indices is $\mathcal{L}_{\mathrm{i}}=\{1,2,3,4,5\}$ and we are determining $\left|P_{\mathcal{L}_{\mathrm{i}}}\left(\left\{x_{k}\right\},\left\{m_{k}\right\} ;\left\{t_{k}\right\}\right)\right\rangle$. At the end of the first epoch, only the times $t_{3}, t_{4}$ are larger than the renewal time, therefore our set of indices is $\mathcal{L}_{\mathrm{f}}=\{3,4\}$. We have to describe what happens in this first epoch while for the rest of the process, we can use the renewal time as a shifted zero point and use $\left|P_{\mathcal{L}_{\mathrm{f}}}\left(\left\{x_{k}\right\},\left\{m_{k}\right\} ;\left\{t_{k}-\tau_{1}\right\}\right)\right\rangle$.

Noting here

$$
\begin{aligned}
\left(\prod_{k \in \mathcal{L}_{\mathrm{i}}} \int_{0}^{\infty} \mathrm{d} t_{k} e^{-\lambda_{k} t_{k}}\right) \prod_{j \in \mathcal{L}_{\mathrm{i}} \backslash \mathcal{L}_{\mathrm{f}}} \theta\left(\tau-t_{j}\right) \prod_{j \in \mathcal{L}_{\mathrm{f}}} \delta\left(\tau-t_{j}\right) & =\exp \left(-\Lambda_{\mathcal{L}_{\mathrm{f}}} \tau\right) \prod_{j \in \mathcal{L}_{\mathrm{i}} \backslash \mathcal{L}_{\mathrm{f}}} \frac{1-e^{-\lambda_{j} \tau}}{\lambda_{j}} \\
& =\frac{1}{\prod_{j \in \mathcal{L}_{\mathrm{i}} \backslash \mathcal{L}_{\mathrm{f}}} \lambda_{j}} \sum_{\mathcal{J}: \mathcal{L}_{\mathrm{i}} \supseteq \mathcal{J} \supseteq \mathcal{L}_{\mathrm{f}}}(-1)^{\left|\mathcal{J} \backslash \mathcal{L}_{\mathrm{f}}\right|} \exp \left(-\Lambda_{\mathcal{J}} \tau\right)
\end{aligned}
$$

where $\left|\mathcal{J} \backslash \mathcal{L}_{\mathrm{f}}\right|$ denotes the number of elements in the set $\mathcal{J} \backslash \mathcal{L}_{\mathrm{f}}$. Therefore $(-1)^{\left|\mathcal{J} \backslash \mathcal{L}_{\mathrm{f}}\right|}$ is 1 if $\mathcal{J} \backslash \mathcal{L}_{\mathrm{f}}$ has an even number of elements and it is -1 for an odd number. Additionally, $\sum_{\mathcal{J}: \mathcal{L}_{\mathrm{i}} \supseteq \mathcal{J} \supseteq \mathcal{L}_{\mathrm{f}}}$ denotes a sum over all subsets $\mathcal{J}$ of $\mathcal{L}_{\mathrm{i}}$ which contain $\mathcal{L}_{\mathrm{f}}$. Use the situation in Fig. 3 as example with $\mathcal{L}_{\mathrm{i}}=\{1,2,3,4\}$ and $\mathcal{L}_{\mathrm{f}}=\{2,3\}$ we sum over $\mathcal{J}=\{2,3\}, \mathcal{J}=\{1,2,3\}, \mathcal{J}=\{2,3,4\}$ and $\mathcal{J}=\{1,2,3,4\}$ :

$$
\begin{aligned}
\frac{1}{\prod_{j \in \mathcal{L}_{\mathrm{i}} \backslash \mathcal{L}_{\mathrm{f}}} \lambda_{j}} & \sum_{\mathcal{J}: \mathcal{L}_{\mathrm{i}} \supseteq \mathcal{J} \supseteq \mathcal{L}_{\mathrm{f}}}(-1)^{\left|\mathcal{J} \backslash \mathcal{L}_{\mathrm{f}}\right|} \exp \left(-\Lambda_{\mathcal{J}} \tau\right) \\
& =\frac{1}{\prod_{j \in\{1,4\}} \lambda_{j}} \sum_{\mathcal{J}:\{2,3\} \supseteq \mathcal{J} \supseteq\{1,2,3,4\}}(-1)^{\left|\mathcal{J} \backslash \mathcal{L}_{\mathrm{f}}\right|} \exp \left(-\Lambda_{\mathcal{J}} \tau\right) \\
& =\frac{1}{\lambda_{1} \lambda_{4}}\left(\exp \left(-\Lambda_{\{2,3\}} \tau\right)-\exp \left(-\Lambda_{\{1,2,3\}} \tau\right)-\exp \left(-\Lambda_{\{2,3,4\}} \tau\right)+\exp \left(-\Lambda_{\{1,2,3,4\}} \tau\right)\right)
\end{aligned}
$$


Using the formula Eq. (3.12) in Eq. (3.11) gives

$$
\begin{aligned}
\hat{Q}_{\mathcal{L}_{\mathrm{i}} \mathcal{L}_{\mathrm{f}}} & \left\{\left\{x_{k}\right\},\left\{m_{k}\right\} ;\left\{\lambda_{k}\right\}\right) \\
= & \frac{1}{\prod_{k \in \mathcal{L}_{\mathrm{i}} \backslash \mathcal{L}_{\mathrm{f}}} \lambda_{k}} \sum_{l}|l\rangle\langle l| \int_{-\infty}^{\infty} \mathrm{d} \chi \prod_{k \in \mathcal{L}_{\mathrm{i}} \backslash \mathcal{L}_{\mathrm{f}}} \delta_{l m_{k}} \delta\left(\chi-x_{k}\right) \sum_{\mathcal{J}: \mathcal{L}_{\mathrm{i}} \supseteq \mathcal{J} \supseteq \mathcal{L}_{\mathrm{f}}}(-1)^{\left|\mathcal{J} \backslash \mathcal{L}_{\mathrm{f}}\right| \hat{\Phi}\left(\chi, \Lambda_{\mathcal{J}}\right) M .}
\end{aligned}
$$

Putting these results into Eq. (3.8) gives in Laplace space

$$
\left|\hat{P}_{\mathcal{L}_{\mathrm{i}}}\left(\left\{x_{k}\right\},\left\{m_{k}\right\} ;\left\{\lambda_{k}\right\}\right)\right\rangle=\sum_{\mathcal{L}_{\mathrm{f}}: \mathcal{L}_{\mathrm{i}} \supseteq \mathcal{L}_{\mathrm{f}}} \hat{Q}_{\mathcal{L}_{\mathrm{i}} \mathcal{L}_{\mathrm{f}}}\left(\left\{x_{k}\right\},\left\{m_{k}\right\} ;\left\{\lambda_{k}\right\}\right)\left|\hat{P}_{\mathcal{L}_{\mathrm{f}}}\left(\left\{x_{k}\right\},\left\{m_{k}\right\} ;\left\{\lambda_{k}\right\}\right)\right\rangle .
$$

This is not yet a real recursion formula, as we have $\left|\hat{P}_{\mathcal{L}_{\mathrm{i}}}\left(\left\{x_{k}\right\},\left\{m_{k}\right\} ;\left\{\lambda_{k}\right\}\right)\right\rangle$ on both sides. For $\mathcal{L}_{\mathrm{f}}=\mathcal{L}_{\mathrm{i}}$ we have

$$
\hat{Q}_{\mathcal{L}_{\mathrm{i}} \mathcal{L}_{\mathrm{i}}}\left(\left\{x_{k}\right\},\left\{m_{k}\right\} ;\left\{\lambda_{k}\right\}\right)=\hat{\Psi}_{0}\left(\Lambda_{\mathcal{L}_{\mathrm{i}}}\right) M
$$

such that we can reformulate Eq. (3.15) to contain a sum over the true subsets $\mathcal{L}_{\mathrm{f}}$ of $\mathcal{L}_{\mathrm{i}}$, i.e., $\mathcal{L}_{\mathrm{f}}$ is a subset of $\mathcal{L}_{\mathrm{f}}$ and $\mathcal{L}_{\mathrm{i}} \neq \mathcal{L}_{\mathrm{f}}\left(\right.$ notation: $\left.\mathcal{L}_{\mathrm{i}} \supsetneq \mathcal{L}_{\mathrm{f}}\right)$ :

$$
\begin{aligned}
\left|\hat{P}_{\mathcal{L}_{\mathrm{i}}}\left(\left\{x_{k}\right\},\left\{m_{k}\right\} ;\left\{\lambda_{k}\right\}\right)\right\rangle= & \hat{\Psi}_{0}\left(\Lambda_{\mathcal{L}_{\mathrm{i}}}\right) M\left|\hat{P}_{\mathcal{L}_{\mathrm{i}}}\left(\left\{x_{k}\right\},\left\{m_{k}\right\} ;\left\{\lambda_{k}\right\}\right)\right\rangle \\
& +\sum_{\mathcal{L}_{\mathrm{f}}: \mathcal{L}_{\mathrm{i}} \supsetneq \mathcal{L}_{\mathrm{f}}} \hat{Q}_{\mathcal{L}_{\mathrm{i}} \mathcal{L}_{\mathrm{f}}}\left(\left\{x_{k}\right\},\left\{m_{k}\right\} ;\left\{\lambda_{k}\right\}\right)\left|\hat{P}_{\mathcal{L}_{\mathrm{f}}}\left(\left\{x_{k}\right\},\left\{m_{k}\right\} ;\left\{\lambda_{k}\right\}\right)\right\rangle
\end{aligned}
$$

and therefore

$$
\left|\hat{P}_{\mathcal{L}_{\mathrm{i}}}\left(\left\{x_{k}\right\},\left\{m_{k}\right\} ;\left\{\lambda_{k}\right\}\right)\right\rangle=\left[\mathbf{I}-\hat{\Psi}_{0}\left(\Lambda_{\mathcal{L}_{\mathrm{i}}}\right) M\right]^{-1} \sum_{\mathcal{L}_{\mathrm{f}}: \mathcal{L}_{\mathrm{i}} \supsetneq \mathcal{L}_{\mathrm{f}}} \hat{Q}_{\mathcal{L}_{\mathrm{i}} \mathcal{L}_{\mathrm{f}}}\left(\left\{x_{k}\right\},\left\{m_{k}\right\} ;\left\{\lambda_{k}\right\}\right)\left|\hat{P}_{\mathcal{L}_{\mathrm{f}}}\left(\left\{x_{k}\right\},\left\{m_{k}\right\} ;\left\{\lambda_{k}\right\}\right)\right\rangle .
$$

A graphical explanation of the terms of Eq. (3.18) is given in Figure 3. This formula is the main result of this section.

\subsection{Examples}

Using Eq. (3.18) gives for the one-time probability density

$$
\left|\hat{P}_{1}(x, m ; \lambda)\right\rangle=\left[\mathbf{I}-\hat{\Psi}_{0}(\lambda) M\right]^{-1} \frac{\hat{\Phi}(x, 0)-\hat{\Phi}(x, \lambda)}{\lambda}|m\rangle .
$$

And for the two-time probability density

$$
\begin{aligned}
\left|\hat{P}_{2}\left(\left\{x_{1}, x_{2}\right\},\left\{m_{1}, m_{2}\right\} ;\left\{\lambda_{1}, \lambda_{2}\right\}\right)\right\rangle \\
=\left[\mathbf{I}-\hat{\Psi}_{0}\left(\lambda_{1}+\lambda_{2}\right) M\right]^{-1} \frac{\hat{\Phi}\left(x_{1}, \lambda_{2}\right)-\hat{\Phi}\left(x_{1}, \lambda_{1}+\lambda_{2}\right)}{\lambda_{1}}\left|m_{1}\right\rangle\left\langle m_{1}|M| \hat{P}_{1}\left(x_{2}, m_{2} ; \lambda_{2}\right)\right\rangle \\
\quad+\left[\mathbf{I}-\hat{\Psi}_{0}\left(\lambda_{1}+\lambda_{2}\right) M\right]^{-1} \frac{\hat{\Phi}\left(x_{2}, \lambda_{1}\right)-\hat{\Phi}\left(x_{2}, \lambda_{1}+\lambda_{2}\right)}{\lambda_{2}}\left|m_{2}\right\rangle\left\langle m_{2}|M| \hat{P}_{1}\left(x_{1}, m_{1} ; \lambda_{1}\right)\right\rangle \\
\quad+\left[\mathbf{I}-\hat{\Psi}_{0}\left(\lambda_{1}+\lambda_{2}\right) M\right]^{-1} \frac{\hat{\Phi}\left(x_{1}, 0\right)-\hat{\Phi}\left(x_{1}, \lambda_{1}\right)-\hat{\Phi}\left(x_{1}, \lambda_{2}\right)+\hat{\Phi}\left(x_{1}, \lambda_{1}+\lambda_{2}\right)}{\lambda_{1} \lambda_{2}} \delta\left(x_{1}-x_{2}\right) \delta_{m_{1} m_{2}}\left|m_{1}\right\rangle .
\end{aligned}
$$

For the single state model, we get (observing, that all vectors are one-dimensional and need not to be written out)

$$
\hat{P}_{1}(x ; \lambda)=\frac{1}{\lambda} \frac{\hat{\phi}^{\text {single }}(x, 0)-\hat{\phi}^{\text {single }}(x, \lambda)}{1-\hat{\psi}_{0}^{\text {single }}(\lambda)}
$$




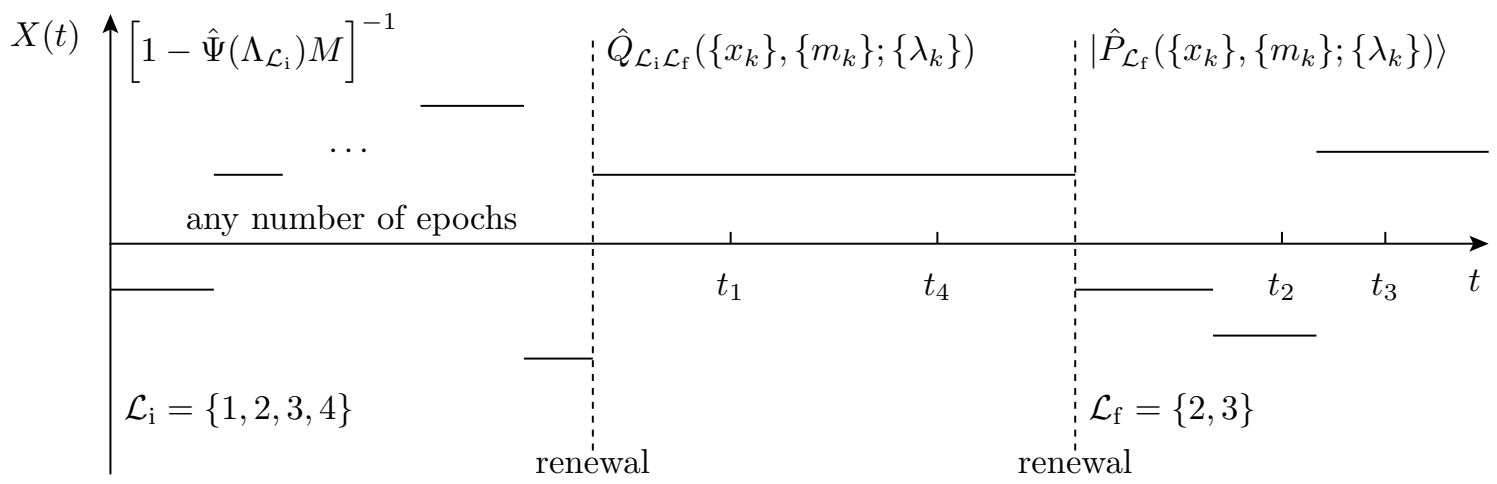

Figure 3. Graphical explanation of Eq. (3.18): This is one term in the determination of $\left|\hat{P}_{\mathcal{L}_{\mathrm{i}}}\left(\left\{x_{k}\right\},\left\{m_{k}\right\} ;\left\{\lambda_{k}\right\}\right)\right\rangle$ with $\mathcal{L}_{\mathrm{i}}=\{1,2,3,4\}$, i.e., we are interested in a four-time joint probability distribution at the times $t_{1}, t_{2}, t_{3}, t_{4}$. The term $\left[\mathbf{I}-\hat{\Psi}_{0}\left(\Lambda_{\mathcal{L}_{\mathrm{i}}}\right) M\right]^{-1}$ accounts for any number of epochs which are completely before any of the times $t_{1}, t_{2}, t_{3}, t_{4}$. Then the first epoch containing one or more of the times $t_{1}, t_{2}, t_{3}, t_{4}$ arrives: in our example, it contains the time $t_{1}$ and $t_{4}$ and it is confined by the two dashed lines. The set $\mathcal{L}_{\mathrm{f}}$ describes the times after the end of this epoch - here $\mathcal{L}_{\mathrm{f}}=\{2,3\}$. The contribution of this epoch is given by $\hat{Q}_{\mathcal{L}_{\mathrm{i}} \mathcal{L}_{\mathrm{f}}}\left(\left\{x_{k}\right\},\left\{m_{k}\right\} ;\left\{\lambda_{k}\right\}\right)$. At the end of this epoch we can use the renewal property to describe the rest of the process with the two-time joint probability distribution $\left|\hat{P}_{\mathcal{L}_{\mathrm{f}}}\left(\left\{x_{k}\right\},\left\{m_{k}\right\} ;\left\{\lambda_{k}\right\}\right)\right\rangle$ containing only the time $t_{2}$ and $t_{3}$. The complete $\left|\hat{P}_{\mathcal{L}_{\mathrm{i}}}\left(\left\{x_{k}\right\},\left\{m_{k}\right\} ;\left\{\lambda_{k}\right\}\right)\right\rangle$ is obtained by summing over all true subsets $\mathcal{L}_{\mathrm{f}}$ of $\mathcal{L}_{\mathrm{i}}$ (denoted $\left.\mathcal{L}_{\mathrm{f}} \subsetneq \mathcal{L}_{\mathrm{i}}\right)$.

and

$$
\begin{aligned}
P_{2}\left(\left\{x_{1}, x_{2}\right\} ;\left\{\lambda_{1}, \lambda_{2}\right\}\right)= & \frac{1}{\lambda_{1}} \frac{\hat{\phi}^{\text {single }}\left(x_{1}, \lambda_{2}\right)-\hat{\phi}^{\text {single }}\left(x_{1}, \lambda_{1}+\lambda_{2}\right)}{1-\hat{\psi}_{0}^{\text {single }}\left(\lambda_{1}+\lambda_{2}\right)} \hat{P}_{1}\left(x_{2} ; \lambda_{2}\right) \\
& +\frac{1}{\lambda_{2}} \frac{\hat{\phi}^{\text {single }}\left(x_{2}, \lambda_{1}\right)-\hat{\phi}^{\text {single }}\left(x_{2}, \lambda_{1}+\lambda_{2}\right)}{1-\hat{\psi}_{0}^{\text {single }}\left(\lambda_{1}+\lambda_{2}\right)} \hat{P}_{1}\left(x_{1} ; \lambda_{1}\right) \\
& +\frac{\delta\left(x_{1}-x_{2}\right)}{\lambda_{1} \lambda_{2}} \frac{\hat{\phi}^{\text {single }}\left(x_{1}, 0\right)-\hat{\phi}^{\text {single }}\left(x_{1}, \lambda_{1}\right)-\hat{\phi}^{\text {single }}\left(x_{1}, \lambda_{2}\right)+\hat{\phi}^{\text {single }}\left(x_{1}, \lambda_{1}+\lambda_{2}\right)}{1-\hat{\psi}_{0}^{\text {single }}\left(\lambda_{1}+\lambda_{2}\right)} .
\end{aligned}
$$

For the alternating model, we get for the one-time probability distribution (using Eq. (2.29))

$$
\begin{aligned}
& \left|\hat{P}_{1}(x, m ; \lambda)\right\rangle=\frac{1}{1-\hat{\psi}^{\text {alt }}(\lambda)^{2}}\left(\begin{array}{cc}
1 & \hat{\psi}^{\text {alt }}(\lambda) \\
\hat{\psi}^{\text {alt }}(\lambda) & 1
\end{array}\right) \frac{1}{\lambda}\left(\begin{array}{cc}
\delta\left(x-I_{+}\right)\left(1-\hat{\psi}^{\text {alt }}(\lambda)\right) & 0 \\
0 & \delta\left(x-I_{-}\right)\left(1-\hat{\psi}^{\text {alt }}(\lambda)\right)
\end{array}\right)|m\rangle \\
& =\frac{1}{\lambda} \frac{1}{1+\hat{\psi}^{\mathrm{alt}}(\lambda)}\left(\begin{array}{cc}
\delta\left(x-I_{+}\right) & \delta\left(x-I_{-}\right) \hat{\psi}^{\mathrm{alt}}(\lambda) \\
\delta\left(x-I_{+}\right) \hat{\psi}^{\mathrm{alt}}(\lambda) & \delta\left(x-I_{-}\right)
\end{array}\right)|m\rangle \\
& =\frac{1}{\lambda} \frac{\delta\left(x-I_{m}\right)}{1+\hat{\psi}^{\text {alt }}(\lambda)}\left(\begin{array}{cc}
1 & \hat{\psi}^{\text {alt }}(\lambda) \\
\hat{\psi}^{\text {alt }}(\lambda) & 1
\end{array}\right)|m\rangle \text {. }
\end{aligned}
$$

Here, $m$ takes the values $m= \pm$. The elements of the matrix can be interpreted. When we start in a + state, the Laplace transform of $t$ of the probability for being in state + and the density of $X(t)=x$ at 
time $t$ is

$$
\left\langle+\mid \hat{P}_{1}(x,+; \lambda)\right\rangle=\frac{1}{\lambda} \frac{1}{1+\hat{\psi}^{\text {alt }}(\lambda)} \delta\left(x-I_{+}\right)
$$

while we get for being in the - state

$$
\left\langle+\mid \hat{P}_{1}(x,-; \lambda)\right\rangle=\frac{1}{\lambda} \frac{\hat{\psi}^{\text {alt }}(\lambda)}{1+\hat{\psi}^{\text {alt }}(\lambda)} \delta\left(x-I_{-}\right) .
$$

The marginal probability of finding the system at $x$, provided it started in state + is thus

$$
\left\langle+\mid \hat{P}_{1}(x,+; \lambda)\right\rangle+\left\langle+\mid \hat{P}_{1}(x,-; \lambda)\right\rangle=\frac{1}{\lambda} \frac{\delta\left(x-I_{+}\right)+\hat{\psi}^{\text {alt }}(\lambda) \delta\left(x-I_{-}\right)}{1+\hat{\psi}^{\text {alt }}(\lambda)} .
$$

The two time probability becomes

$$
\begin{aligned}
& \left\langle\text { init } \mid \hat{P}_{2}\left(\left\{x_{1}, x_{2}\right\},\left\{m_{1}, m_{2}\right\} ;\left\{\lambda_{1}, \lambda_{2}\right\}\right)\right\rangle \\
& =\frac{\delta\left(x_{1}-I_{m_{1}}\right)}{\lambda_{1}} \frac{\hat{\psi}^{\text {alt }}\left(\lambda_{2}\right)-\hat{\psi}^{\text {alt }}\left(\Lambda_{\{1,2\}}\right)}{1-\hat{\psi}^{\text {alt }}\left(\Lambda_{\{1,2\}}\right)^{2}}\left\langle\operatorname{init}\left|\left(\begin{array}{c}
1 \\
\hat{\psi}^{\text {alt }}\left(\Lambda_{\{1,2\}}\right)
\end{array} \begin{array}{c}
\hat{\psi}^{\text {alt }}\left(\Lambda_{\{1,2\}}\right) \\
1
\end{array}\right)\right| m_{1}\right\rangle\left\langle m_{1}\left|M^{\text {alt }}\right| \hat{P}_{1}\left(x_{2}, m_{2} ; \lambda_{2}\right)\right\rangle \\
& +\frac{\delta\left(x_{2}-I_{m_{2}}\right)}{\lambda_{2}} \frac{\hat{\psi}^{\text {alt }}\left(\lambda_{1}\right)-\hat{\psi}^{\text {alt }}\left(\Lambda_{\{1,2\}}\right)}{1-\hat{\psi}^{\text {alt }}\left(\Lambda_{\{1,2\}}\right)^{2}}\left\langle\operatorname{init}\left|\left(\begin{array}{c}
1 \\
\hat{\psi}^{\text {alt }}\left(\Lambda_{\{1,2\}}\right)
\end{array} \hat{\psi}^{\text {alt }}\left(\Lambda_{\{1,2\}}\right)\right)\right| m_{2}\right\rangle \\
& \times\left\langle m_{2}\left|M^{\text {alt }}\right| \hat{P}_{1}\left(x_{1}, m_{1} ; \lambda_{1}\right)\right\rangle \\
& +\frac{\delta\left(x_{1}-I_{m_{1}}\right) \delta\left(x_{2}-I_{m_{2}}\right)}{\lambda_{1} \lambda_{2}} \frac{1-\hat{\psi}^{\text {alt }}\left(\lambda_{1}\right)-\hat{\psi}^{\text {alt }}\left(\lambda_{2}\right)+\hat{\psi}^{\text {alt }}\left(\Lambda_{\{1,2\}}\right)}{1-\hat{\psi}^{\text {alt }}\left(\Lambda_{\{1,2\}}\right)^{2}} \delta_{m_{1} m_{2}} \\
& \times\left\langle\operatorname{init}\left|\left(\begin{array}{cc}
1 & \hat{\psi}^{\text {alt }}\left(\Lambda_{\{1,2\}}\right) \\
\hat{\psi}^{\text {alt }}\left(\Lambda_{\{1,2\}}\right) & 1
\end{array}\right)\right| m_{1}\right\rangle .
\end{aligned}
$$

The probability for starting in state + and - with same probability and then having $m_{1}=+$ and $m_{2}=-$ at times $t_{1}, t_{2}$ in Laplace space is $\left(\langle-| M^{\text {alt }}=\langle+|\right.$ and $\left.\langle+| M^{\text {alt }}=\langle-|\right)$

$$
\begin{aligned}
\int_{-\infty}^{\infty} \mathrm{d} x_{1} \int_{-\infty}^{\infty} \mathrm{d} x_{2}\left\langle\mathrm{eq}_{\mathrm{M}}\right| & \left.\hat{P}_{2}\left(\left\{x_{1}, x_{2}\right\},\left\{m_{1}=+, m_{2}=-\right\} ;\left\{\lambda_{1}, \lambda_{2}\right\}\right)\right\rangle \\
= & \frac{1}{2 \lambda_{1}} \frac{\hat{\psi}^{\text {alt }}\left(\lambda_{2}\right)-\hat{\psi}^{\text {alt }}\left(\Lambda_{\{1,2\}}\right)}{1-\hat{\psi}^{\text {alt }}\left(\Lambda_{\{1,2\}}\right)} \int_{-\infty}^{\infty} \mathrm{d} x_{2}\left\langle+\left|M^{\text {alt }}\right| \hat{P}_{1}\left(x_{2}, m_{2}=-; \lambda_{2}\right)\right\rangle \\
& +\frac{1}{2 \lambda_{2}} \frac{\hat{\psi}^{\text {alt }}\left(\lambda_{1}\right)-\hat{\psi}^{\text {alt }}\left(\Lambda_{\{1,2\}}\right)}{1-\hat{\psi}^{\text {alt }}\left(\Lambda_{\{1,2\}}\right)} \int_{-\infty}^{\infty} \mathrm{d} x_{1}\left\langle-\left|M^{\text {alt }}\right| \hat{P}_{1}\left(x_{1}, m_{1}=+; \lambda_{1}\right)\right\rangle \\
= & \frac{1}{\lambda_{1} \lambda_{2}} \frac{1}{1-\hat{\psi}^{\text {alt }}\left(\Lambda_{\{1,2\}}\right)} \frac{1}{2}\left(\frac{\hat{\psi}^{\text {alt }}\left(\lambda_{2}\right)-\hat{\psi}^{\text {alt }}\left(\Lambda_{\{1,2\}}\right)}{1+\hat{\psi}^{\text {alt }}\left(\lambda_{2}\right)}+\frac{\hat{\psi}^{\text {alt }}\left(\lambda_{1}\right)-\hat{\psi}^{\text {alt }}\left(\Lambda_{\{1,2\}}\right)}{1+\hat{\psi}^{\text {alt }}\left(\lambda_{1}\right)}\right)
\end{aligned}
$$

Here, having $m_{1}=+$ implies $X\left(t_{1}\right)=I_{+}$and $m_{2}=-\operatorname{implies} X\left(t_{2}\right)=I_{-}$.

\section{The Aged Process}

\subsection{Forward Recurrence Time}

Sometimes the observed process does not start at a renewal but a time $t_{\mathrm{a}}$ after a renewal [4,38]. This could happen if the measurement starts a time $t_{\mathrm{a}}$ after a the process starts (independent, if meanwhile 
renewals did happen or not). Formally this is done by starting the process at time $-t_{\mathrm{a}}$. We can use the stochastic process $X(t)$ by a shift of time

$$
X^{\text {aged }}(t)=X\left(t+t_{\mathrm{a}}\right) .
$$

It will prove useful to additionally keep track of the distribution of $X\left(t_{\mathrm{a}}\right)$ and $N\left(t_{\mathrm{a}}\right)$ at the beginning of the measurement whose distribution are denoted by the variables $y$ and $n$. Formally

$$
\left\langle\operatorname{init} \mid P_{\mathcal{L}}^{\text {aged }}\left(\left\{x_{k}\right\},\left\{m_{k}\right\}, y, n ;\left\{t_{k}\right\}, t_{\mathrm{a}}\right)\right\rangle=\left\langle\delta\left(X\left(t_{\mathrm{a}}\right)-y\right) \delta_{N\left(t_{\mathrm{a}}\right) n} \prod_{k \in \mathcal{L}} \delta\left(X\left(t_{k}+t_{\mathrm{a}}\right)-x_{k}\right) \delta_{N\left(t_{k}+t_{\mathrm{a}}\right) m_{k}}\right\rangle_{\langle\text {init }|}
$$

with the Laplace transform in the $\left\{t_{k}\right\}$ (with Laplace duals $\left\{\lambda_{k}\right\}$ ) and $t_{\mathrm{a}}$ (with Laplace dual $s_{\mathrm{a}}$ )

$$
\left|\hat{P}_{\mathcal{L}}^{\text {aged }}\left(\left\{x_{k}\right\},\left\{m_{k}\right\}, y, n ;\left\{\lambda_{k}\right\}, s_{\mathrm{a}}\right)\right\rangle=\int_{0}^{\infty} \mathrm{d} t_{\mathrm{a}} e^{-s_{\mathrm{a}} t_{\mathrm{a}}}\left(\prod_{k \in \mathcal{L}} \int_{0}^{\infty} \mathrm{d} t_{k} e^{-\lambda_{k} t_{k}}\right)\left|P_{\mathcal{L}}^{\text {aged }}\left(\left\{x_{k}\right\},\left\{m_{k}\right\}, y, n ;\left\{t_{k}\right\}, t_{\mathrm{a}}\right)\right\rangle
$$

In a first step we determine the forward recurrence time [20]. We consider a process which starts in a state $i$ and evolves a time $t_{\mathrm{a}}$. We want to determine the joint probability that the process is in state $j$ with the probability density that the process takes the value $x$ and the time to the next renewal (the forward renewal time) is $t_{\mathrm{f}}$. We denote this with help of a matrix $\Phi^{\mathrm{fw}}\left(x, t_{\mathrm{f}} ; t_{\mathrm{a}}\right)$ :

$$
\left\langle i\left|\Phi^{\mathrm{fw}}\left(x, t_{\mathrm{f}} ; t_{\mathrm{a}}\right)\right| j\right\rangle \text {. }
$$

For any initial distribution $\left\langle\right.$ init| (at time $-t_{\mathrm{a}}$ ) we have the probability to be in state $i$ at the beginning $\langle$ init $\mid i\rangle$, so that the above joint probability for the initial distribution 〈init| is

$$
\sum_{i}\langle\operatorname{init} \mid i\rangle\left\langle i\left|\Phi^{\mathrm{fw}}\left(x, t_{\mathrm{f}} ; t_{\mathrm{a}}\right)\right| j\right\rangle=\left\langle\operatorname{init}\left|\Phi^{\mathrm{fw}}\left(x, t_{\mathrm{f}} ; t_{\mathrm{a}}\right)\right| j\right\rangle .
$$

The determination of $\Phi^{\mathrm{fw}}\left(x, t_{\mathrm{f}} ; t_{\mathrm{a}}\right)$ can be done with a renewal ansatz: if $\tau$ denotes the time of the first recurrence, we can split the probability in the two cases $\tau \leq t_{\mathrm{a}}$ and $\tau>t_{\mathrm{a}}$. The case $\tau<t_{\mathrm{a}}$ we have a renewal at time $\tau$, after this the process starts again with probability $\langle i|M| k\rangle$ in state $k$ - in this case the probability distribution is described by $\left\langle k\left|\Phi^{\mathrm{fw}}\left(x, t_{\mathrm{f}} ; t_{\mathrm{a}}-\tau\right)\right| j\right\rangle$. In the case $\tau>t_{\mathrm{a}}$ the distribution is described by the residual time $t_{\mathrm{f}}=\tau-t_{\mathrm{a}}$ while $j$ must be equal to $i$ and $x$ is described by the current state. Put in formula

$$
\left\langle i\left|\Phi^{\mathrm{fw}}\left(x, t_{\mathrm{f}} ; t_{\mathrm{a}}\right)\right| j\right\rangle=\int_{0}^{t_{\mathrm{a}}} \mathrm{d} \tau \sum_{k}\left\langle i\left|\Psi_{0}(\tau) M\right| k\right\rangle\left\langle k\left|\Phi^{\mathrm{fw}}\left(x, t_{\mathrm{f}} ; t_{\mathrm{a}}-\tau\right)\right| j\right\rangle+\int_{t_{\mathrm{a}}}^{\infty} \mathrm{d} \tau\left\langle i\left|\Phi(x, \tau) \delta\left(t_{\mathrm{f}}-\left(\tau-t_{\mathrm{a}}\right)\right)\right| j\right\rangle .
$$

Since $i$ and $j$ are arbitrary and $\sum_{k}|k\rangle\langle k|=\mathbf{I}$, we get

$$
\begin{aligned}
\Phi^{\mathrm{fw}}\left(x, t_{\mathrm{f}} ; t_{\mathrm{a}}\right) & =\int_{0}^{t_{\mathrm{a}}} \mathrm{d} \tau \Psi_{0}(\tau) M \Phi^{\mathrm{fw}}\left(x, t_{\mathrm{f}} ; t_{\mathrm{a}}-\tau\right)+\int_{t_{\mathrm{a}}}^{\infty} \mathrm{d} \tau \Phi(x, \tau) \delta\left(t_{\mathrm{f}}-\left(\tau-t_{\mathrm{a}}\right)\right) \\
& =\int_{0}^{t_{\mathrm{a}}} \mathrm{d} \tau \Psi_{0}(\tau) M \Phi^{\mathrm{fw}}\left(x, t_{\mathrm{f}} ; t_{\mathrm{a}}-\tau\right)+\Phi\left(x, t_{\mathrm{f}}+t_{\mathrm{a}}\right) .
\end{aligned}
$$

Taking the double Laplace transform

$$
\hat{\Phi}^{\mathrm{fw}}\left(x, \lambda_{\mathrm{f}} ; s_{\mathrm{a}}\right)=\int_{0}^{\infty} \mathrm{d} t_{\mathrm{f}} \int_{0}^{\infty} \mathrm{d} t_{\mathrm{a}} \exp \left(-\lambda_{\mathrm{f}} t_{\mathrm{f}}-s_{\mathrm{a}} t_{\mathrm{a}}\right) \Phi^{\mathrm{fw}}\left(x, t_{\mathrm{f}} ; t_{\mathrm{a}}\right)
$$


and applying the transform on Eq. (4.7) gives

$$
\begin{aligned}
\hat{\Phi}^{\mathrm{fw}}\left(x, \lambda_{\mathrm{f}} ; s_{\mathrm{a}}\right) & =\hat{\Psi}_{0}\left(s_{\mathrm{a}}\right) M \hat{\Phi}^{\mathrm{fw}}\left(x, \lambda_{\mathrm{f}} ; s_{\mathrm{a}}\right)+\int_{0}^{\infty} \mathrm{d} t_{\mathrm{f}} \int_{0}^{\infty} \mathrm{d} t_{\mathrm{a}} \exp \left(-\lambda_{\mathrm{f}} t_{\mathrm{f}}-s_{\mathrm{a}} t_{\mathrm{a}}\right) \Phi\left(x, t_{\mathrm{f}}+t_{\mathrm{a}}\right) \\
& =\hat{\Psi}_{0}\left(s_{\mathrm{a}}\right) M \hat{\Phi}^{\mathrm{fw}}\left(x, \lambda_{\mathrm{f}} ; s_{\mathrm{a}}\right)+\int_{0}^{\infty} \mathrm{d} \tilde{t} \frac{e^{-\lambda_{\mathrm{f}} \tilde{t}}-e^{-s_{\mathrm{a}} \tilde{t}}}{s_{\mathrm{a}}-\lambda_{\mathrm{f}}} \Phi(x, \tilde{t}) \\
& =\hat{\Psi}_{0}\left(s_{\mathrm{a}}\right) M \hat{\Phi}^{\mathrm{fw}}\left(x, \lambda_{\mathrm{f}} ; s_{\mathrm{a}}\right)+\frac{1}{s_{\mathrm{a}}-\lambda_{\mathrm{f}}}\left(\hat{\Phi}\left(x, \lambda_{\mathrm{f}}\right)-\hat{\Phi}\left(x, s_{\mathrm{a}}\right)\right) .
\end{aligned}
$$

Solving this for $\hat{\Phi}^{\mathrm{fw}}\left(x, \lambda_{\mathrm{f}} ; s_{\mathrm{a}}\right)$ gives

$$
\hat{\Phi}^{\mathrm{fw}}\left(x, \lambda_{\mathrm{f}} ; s_{\mathrm{a}}\right)=\frac{1}{s_{\mathrm{a}}-\lambda_{\mathrm{f}}}\left[\mathbf{I}-\hat{\Psi}_{0}\left(s_{\mathrm{a}}\right) M\right]^{-1}\left(\hat{\Phi}\left(x, \lambda_{\mathrm{f}}\right)-\hat{\Phi}\left(x, s_{\mathrm{a}}\right)\right) .
$$

The $x$ moments are then

$$
\begin{aligned}
\hat{\Psi}_{n}^{\mathrm{fw}}\left(\lambda_{\mathrm{f}} ; s_{\mathrm{a}}\right) & =\int \mathrm{d} x x^{n} \hat{\Phi}^{\mathrm{fw}}\left(x, \lambda_{\mathrm{f}} ; s_{\mathrm{a}}\right) \\
& =\frac{1}{s_{\mathrm{a}}-\lambda_{\mathrm{f}}}\left[\mathbf{I}-\hat{\Psi}_{0}\left(s_{\mathrm{a}}\right) M\right]^{-1}\left(\hat{\Psi}_{n}\left(\lambda_{\mathrm{f}}\right)-\hat{\Psi}_{n}\left(s_{\mathrm{a}}\right)\right) .
\end{aligned}
$$

We will provide examples below.

\subsection{Probability Distributions}

We have to look again at the epoch containing $t_{\mathrm{a}}$. In this time the waiting time till the first renewal is described by the forward waiting time. Starting from this renewal, the rest of the process is described by the probability distribution of an process without ageing. Completely analogous to Eq. (3.8) we obtain

$$
\begin{aligned}
\mid P_{\mathcal{L}_{\mathrm{i}}}^{\text {aged }} & \left.\left(\left\{x_{k}\right\},\left\{m_{k}\right\}, y, n ;\left\{t_{k}\right\}, t_{\mathrm{a}}\right)\right\rangle \\
= & \sum_{\mathcal{L}_{\mathrm{f}}: \mathcal{L}_{\mathrm{i}} \supseteq \mathcal{L}_{\mathrm{f}}} \int_{0}^{\infty} \mathrm{d} \tau \int_{-\infty}^{\infty} \mathrm{d} \chi \delta(\chi-y)\left(\prod_{j \in \mathcal{L}_{\mathrm{i}} \backslash \mathcal{L}_{\mathrm{f}}} \delta_{n m_{j}} \delta\left(\chi-x_{j}\right) \theta\left(\tau-t_{j}\right)\right) \\
& \times \Phi^{\mathrm{fw}}(\chi, \tau)|n\rangle\left\langle n|M| P_{\mathcal{L}_{\mathrm{f}}}\left(\left\{x_{k}\right\},\left\{m_{k}\right\} ;\left\{t_{k}-\tau\right\}\right)\right\rangle .
\end{aligned}
$$

Transforming the time variables to Laplace space (the $\left\{t_{k}\right\}$ s and $t_{\mathrm{a}}$ ) and following exactly the calculations done in Sec. 3, we get a step operator

$$
\begin{aligned}
\hat{Q}_{\mathcal{L}_{\mathrm{i}} \mathcal{L}_{\mathrm{f}}}^{\text {aged }} & \left(\left\{x_{k}\right\},\left\{m_{k}\right\}, y, n ;\left\{\lambda_{k}\right\} ; s_{\mathrm{a}}\right) \\
& =\frac{1}{\prod_{k \in \mathcal{L}_{\mathrm{i}} \backslash \mathcal{L}_{\mathrm{f}}} \lambda_{k}} \int_{-\infty}^{\infty} \mathrm{d} \chi \delta(\chi-y) \prod_{k \in \mathcal{L}_{\mathrm{i}} \backslash \mathcal{L}_{\mathrm{f}}} \delta_{n m_{k}} \delta\left(\chi-x_{k}\right) \sum_{\mathcal{J}: \mathcal{L}_{\mathrm{i}} \supseteq \mathcal{J} \supseteq \mathcal{L}_{\mathrm{f}}}(-1)^{\left|\mathcal{J} \backslash \mathcal{L}_{\mathrm{f}}\right|} \hat{\Phi}^{\mathrm{fw}}\left(\chi, \Lambda_{\mathcal{J}} ; s_{\mathrm{a}}\right)|n\rangle\langle n| M .
\end{aligned}
$$

and the relation

$$
\left|\hat{P}_{\mathcal{L}_{\mathrm{i}}}^{\text {aged }}\left(\left\{x_{k}\right\},\left\{m_{k}\right\}, y, n ;\left\{\lambda_{k}\right\}, s_{\mathrm{a}}\right)\right\rangle=\sum_{\mathcal{L}_{\mathrm{f}}: \mathcal{L}_{\mathrm{i}} \supseteq \mathcal{L}_{\mathrm{f}}} \hat{Q}_{\mathcal{L}_{\mathrm{i}} \mathcal{L}_{\mathrm{f}}}^{\text {aged }}\left(\left\{x_{k}\right\},\left\{m_{k}\right\}, y, n ;\left\{\lambda_{k}\right\}, s_{\mathrm{a}}\right)\left|\hat{P}_{\mathcal{L}_{\mathrm{f}}}\left(\left\{x_{k}\right\},\left\{m_{k}\right\} ;\left\{\lambda_{k}\right\}\right)\right\rangle .
$$

\subsection{Examples}

Using Eq. (4.14) gives for the one-time probability density

$$
\begin{aligned}
\left|\hat{P}_{1}^{\text {aged }}\left(x, m, y, n ; \lambda, s_{\mathrm{a}}\right)\right\rangle= & \hat{\Phi}^{\mathrm{fw}}\left(y, \lambda ; s_{\mathrm{a}}\right)|n\rangle\left\langle n\left|M\left[\mathbf{I}-\hat{\Psi}_{0}(\lambda) M\right]^{-1} \frac{\hat{\Phi}(x, 0)-\hat{\Phi}(x, \lambda)}{\lambda}\right| m\right\rangle \\
& +\frac{\hat{\Phi}^{\mathrm{fw}}\left(x, 0 ; s_{\mathrm{a}}\right)-\hat{\Phi}^{\mathrm{fw}}\left(x, \lambda ; s_{\mathrm{a}}\right)}{\lambda} \delta(x-y) \delta_{n m}|m\rangle .
\end{aligned}
$$


The aged one-time probability density is related to the two-time probability density of the non-aged process. We have

$\left|\hat{P}_{2}\left(\left\{x_{1}, x_{2}\right\},\left\{m_{1}, m_{2}\right\} ;\left\{\lambda_{1}, \lambda_{2}\right\}\right)\right\rangle=\left|P_{1}^{\text {aged }}\left(x_{1}, m_{1}, x_{2}, m_{2} ; \lambda_{1}, \lambda_{1}+\lambda_{2}\right)\right\rangle+\left|P_{1}^{\text {aged }}\left(x_{2}, m_{2}, x_{1}, m_{1} ; \lambda_{2}, \lambda_{1}+\lambda_{2}\right)\right\rangle$

as expected from

$\left|P_{2}\left(\left\{x_{1}, x_{2}\right\},\left\{m_{1}, m_{2}\right\} ;\left\{t_{1}, t_{2}\right\}\right)\right\rangle=\left|P_{1}^{\text {aged }}\left(x_{1}, m_{1}, x_{2}, m_{2} ; t_{1}-t_{2}, t_{2}\right)\right\rangle+\left|P_{1}^{\text {aged }}\left(x_{2}, m_{2}, x_{1}, m_{1} ; t_{2}-t_{1}, t_{1}\right)\right\rangle$

where we defined $\left|P_{1}^{\text {aged }}\left(x_{1}, m_{1}, x_{2}, m_{2} ; t, t_{\mathrm{a}}\right)\right\rangle=0$ for $t<0$ (see also Appendix A for this kind of argument). The two-time aged probability density is

$$
\begin{aligned}
\mid \hat{P}_{2}^{\text {aged }}( & \left.\left.\left\{x_{1}, x_{2}\right\},\left\{m_{1}, m_{2}\right\}, y, n ;\left\{\lambda_{1}, \lambda_{2}\right\}, s_{\mathrm{a}}\right)\right\rangle \\
= & \hat{\Phi}^{\mathrm{fw}}\left(y, \lambda ; s_{\mathrm{a}}\right)|n\rangle\left\langle n|M| \hat{P}_{2}\left(\left\{x_{1}, x_{2}\right\},\left\{m_{1}, m_{2}\right\} ;\left\{\lambda_{1}, \lambda_{2}\right\}\right)\right\rangle \\
& +\frac{\hat{\Phi}^{\mathrm{fw}}\left(x_{1}, \lambda_{2} ; s_{\mathrm{a}}\right)-\hat{\Phi}^{\mathrm{fw}}\left(x_{1}, \lambda_{1}+\lambda_{2} ; s_{\mathrm{a}}\right)}{\lambda_{1}} \delta\left(x_{1}-y\right) \delta_{n m_{1}}\left|m_{1}\right\rangle\left\langle m_{1}|M| \hat{P}_{1}\left(x_{2}, m_{2} ; \lambda_{2}\right)\right\rangle \\
& +\frac{\hat{\Phi}^{\mathrm{fw}}\left(x_{2}, \lambda_{1} ; s_{\mathrm{a}}\right)-\hat{\Phi}^{\mathrm{fw}}\left(x_{2}, \lambda_{1}+\lambda_{2} ; s_{\mathrm{a}}\right)}{\lambda_{2}} \delta\left(x_{2}-y\right) \delta_{n m_{2}}\left|m_{2}\right\rangle\left\langle m_{2}|M| \hat{P}_{1}\left(x_{1}, m_{1} ; \lambda_{1}\right)\right\rangle \\
& +\frac{\hat{\Phi}^{\mathrm{fw}}\left(x_{1}, 0 ; s_{\mathrm{a}}\right)-\hat{\Phi}^{\mathrm{fw}}\left(x_{1}, \lambda_{1} ; s_{\mathrm{a}}\right)-\hat{\Phi}^{\mathrm{fw}}\left(x_{1}, \lambda_{2} ; s_{\mathrm{a}}\right)+\hat{\Phi}^{\mathrm{fw}}\left(x_{1}, \lambda_{1}+\lambda_{2} ; s_{\mathrm{a}}\right)}{\lambda_{1} \lambda_{2}} \\
& \times \delta\left(x_{1}-x_{2}\right) \delta\left(x_{1}-y\right) \delta_{m_{1} m_{2}} \delta_{n m_{1}}\left|m_{1}\right\rangle .
\end{aligned}
$$

For the single state model, the forward density is

$$
\hat{\Phi}^{\mathrm{fw}}\left(x, \lambda_{\mathrm{f}} ; s_{\mathrm{a}}\right)=\frac{1}{s_{\mathrm{a}}-\lambda_{\mathrm{f}}} \frac{\hat{\phi}^{\text {single }}\left(x, \lambda_{\mathrm{f}}\right)-\hat{\phi}^{\text {single }}\left(x, s_{\mathrm{a}}\right)}{1-\hat{\psi}_{0}^{\text {single }}\left(s_{\mathrm{a}}\right)} .
$$

Integrating out the $x$-dependence gives

$$
\int_{-\infty}^{\infty} \mathrm{d} x \hat{\Phi}^{\mathrm{fw}}\left(x, \lambda_{\mathrm{f}} ; s_{\mathrm{a}}\right)=\frac{\hat{\psi}_{0}^{\text {single }}\left(\lambda_{\mathrm{f}}\right)-\hat{\psi}_{0}^{\text {single }}\left(s_{\mathrm{a}}\right)}{s_{\mathrm{a}}-\lambda_{\mathrm{f}}} \frac{1}{1-\hat{\psi}_{0}^{\text {single }}\left(s_{\mathrm{a}}\right)}
$$

which is Eq. (6.2) in [20]. The one-time distribution is

$$
\begin{aligned}
\hat{P}_{1}^{\text {aged }}\left(x, y ; \lambda, s_{\mathrm{a}}\right)= & \frac{1}{s_{\mathrm{a}}-\lambda} \frac{1}{\lambda} \frac{\hat{\phi}^{\text {single }}(y, \lambda)-\hat{\phi}^{\text {single }}\left(y, s_{\mathrm{a}}\right)}{1-\hat{\psi}_{0}^{\text {single }}\left(s_{\mathrm{a}}\right)} \frac{\hat{\phi}^{\text {single }}(x, 0)-\hat{\phi}^{\text {single }}(x, \lambda)}{1-\hat{\psi}_{0}^{\text {single }}(\lambda)} \\
& +\frac{1}{\lambda} \delta(x-y)\left(\frac{1}{s_{\mathrm{a}}} \frac{\hat{\phi}^{\text {single }}(x, 0)-\hat{\phi}^{\text {single }}\left(x, s_{\mathrm{a}}\right)}{1-\hat{\psi}_{0}^{\text {single }}\left(s_{\mathrm{a}}\right)}-\frac{1}{s_{\mathrm{a}}-\lambda} \frac{\hat{\phi}^{\text {single }}(x, \lambda)-\hat{\phi}^{\text {single }}\left(x, s_{\mathrm{a}}\right)}{1-\hat{\psi}_{0}^{\text {single }}\left(s_{\mathrm{a}}\right)}\right) .
\end{aligned}
$$

For the alternating model, the forward density is

$$
\hat{\Phi}^{\mathrm{fw}}\left(x, \lambda_{\mathrm{f}} ; s_{\mathrm{a}}\right)=\frac{1}{s_{\mathrm{a}}-\lambda_{\mathrm{f}}} \frac{\hat{\psi}^{\mathrm{alt}}\left(\lambda_{\mathrm{f}}\right)-\hat{\psi}^{\mathrm{alt}}\left(s_{\mathrm{a}}\right)}{1-\hat{\psi}^{\mathrm{alt}}\left(s_{\mathrm{a}}\right)^{2}}\left(\begin{array}{c}
\delta\left(x-I_{+}\right) \\
\delta\left(x-I_{+}\right) \hat{\psi}^{\mathrm{alt}}\left(s_{\mathrm{a}}\right) \\
\delta\left(x-I_{-}\right) \hat{\psi}^{\mathrm{alt}}\left(s_{\mathrm{a}}\right) \\
\delta\left(x-I_{-}\right)
\end{array}\right) .
$$


The aged distribution becomes (employing additionally the results Eq. (3.23))

$$
\begin{aligned}
& \left\langle\text { init } \mid \hat{P}_{1}^{\text {aged }}\left(x, m, y, n ; \lambda, s_{\mathrm{a}}\right)\right\rangle
\end{aligned}
$$

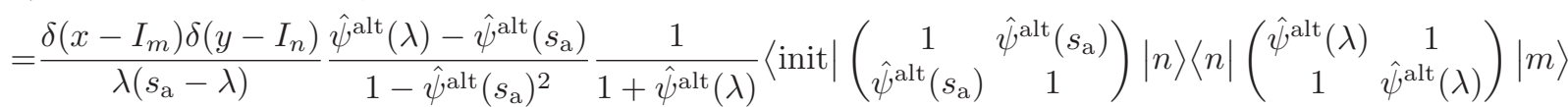

$$
\begin{aligned}
& +\frac{\delta\left(x-I_{m}\right) \delta\left(y-I_{n}\right)}{\lambda s_{\mathrm{a}}} \frac{1}{1+\hat{\psi}^{\mathrm{alt}}\left(s_{\mathrm{a}}\right)}\left\langle\operatorname{init}\left|\left(\begin{array}{cc}
1 & \hat{\psi}^{\mathrm{alt}}\left(s_{\mathrm{a}}\right) \\
\hat{\psi}^{\mathrm{alt}}\left(s_{\mathrm{a}}\right) & 1
\end{array}\right)\right| n\right\rangle\langle n \mid m\rangle
\end{aligned}
$$

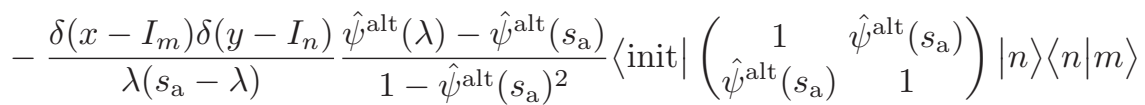

$$
\begin{aligned}
& =\frac{\delta\left(x-I_{m}\right) \delta\left(y-I_{n}\right)}{\lambda\left(s_{\mathrm{a}}-\lambda\right)} \frac{\hat{\psi}^{\text {alt }}(\lambda)-\hat{\psi}^{\text {alt }}\left(s_{\mathrm{a}}\right)}{1-\hat{\psi}^{\text {alt }}\left(s_{\mathrm{a}}\right)^{2}} \frac{1}{1+\hat{\psi}^{\text {alt }}(\lambda)}\left\langle\operatorname{init}\left|\left(\begin{array}{c}
1 \\
\hat{\psi}^{\text {alt }}\left(s_{\mathrm{a}}\right) \\
\hat{\psi}^{\text {alt }}\left(s_{\mathrm{a}}\right) \\
1
\end{array}\right)\right| n\right\rangle\left\langle n\left|\left(\begin{array}{cc}
-1 & 1 \\
1 & -1
\end{array}\right)\right| m\right\rangle \\
& +\frac{\delta\left(x-I_{m}\right) \delta\left(y-I_{n}\right)}{\lambda s_{\mathrm{a}}} \frac{\delta_{m n}}{1+\hat{\psi}^{\text {alt }}\left(s_{\mathrm{a}}\right)}\left\langle\operatorname{init}\left|\left(\begin{array}{cc}
1 & \hat{\psi}^{\text {alt }}\left(s_{\mathrm{a}}\right) \\
\hat{\psi}^{\text {alt }}\left(s_{\mathrm{a}}\right) & 1
\end{array}\right)\right| m\right\rangle
\end{aligned}
$$

The Laplace transform of starting in state + being after time $t_{\mathrm{a}}$ in state - and at time $t_{\mathrm{a}}+t$ in state + is then given by

$$
\int_{-\infty}^{\infty} \mathrm{d} x \int_{-\infty}^{\infty} \mathrm{d} y\left\langle+\mid \hat{P}_{1}^{\mathrm{aged}}\left(x,+, y,-; \lambda, s_{\mathrm{a}}\right)\right\rangle=\frac{1}{\lambda\left(s_{\mathrm{a}}-\lambda\right)} \frac{\hat{\psi}^{\text {alt }}\left(s_{\mathrm{a}}\right)}{1-\left[\hat{\psi}^{\mathrm{alt}}\left(s_{\mathrm{a}}\right)\right]^{2}} \frac{\hat{\psi}^{\text {alt }}(\lambda)-\hat{\psi}^{\text {alt }}\left(s_{\mathrm{a}}\right)}{1+\hat{\psi}^{\text {alt }}(\lambda)} .
$$

\section{The Correlation Functions}

\subsection{Derivation}

We will now obtain the correlation functions $\left\langle X\left(t_{1}\right) \cdots X\left(t_{n}\right)\right\rangle_{\langle\text {init }|}$. We will use these later to obtain the spectrum. In the spirit of the joint probability distributions, we are looking at an arbitrary set of time indices $\mathcal{L}_{\mathrm{i}}$ and define a correlation vector for an arbitrary initial distribution of internal states (init|

$$
\left\langle\operatorname{init} \mid C_{\mathcal{L}_{\mathrm{i}}}\left(\left\{t_{k}\right\}\right)\right\rangle=\left\langle\prod_{j \in \mathcal{L}_{\mathrm{i}}} X\left(t_{j}\right)\right\rangle_{\langle\text {init }|} .
$$

Similar to the probability case, we define the empty correlation function to be

$$
\left|C_{\emptyset}(\{\})\right\rangle=|\Sigma\rangle
$$

which is consistent with the definition of an empty product.

The connection to the last section can be done by

$$
\begin{aligned}
\left\langle\operatorname{init} \mid C_{\mathcal{L}_{\mathrm{i}}}\left(\left\{t_{k}\right\}\right)\right\rangle & =\left\langle\prod_{j \in \mathcal{L}_{\mathrm{i}}} X\left(t_{j}\right)\right\rangle_{\langle\text {init }|} \\
& =\sum_{m_{j}} \prod_{j \in \mathcal{L}_{\mathrm{i}}}\left(\int_{-\infty}^{\infty} \mathrm{d} x_{j} x_{j}\right)\left\langle\text { init } \mid P_{\mathcal{L}_{\mathrm{i}}}\left(\left\{x_{k}\right\},\left\{m_{k}\right\} ;\left\{t_{k}\right\}\right)\right\rangle
\end{aligned}
$$

such that

$$
\left|C_{\mathcal{L}_{\mathrm{i}}}\left(\left\{t_{k}\right\}\right)\right\rangle=\sum_{m_{j}} \prod_{j \in \mathcal{L}_{\mathrm{i}}}\left(\int_{-\infty}^{\infty} \mathrm{d} x_{j} x_{j}\right)\left|P_{\mathcal{L}_{\mathrm{i}}}\left(\left\{x_{k}\right\},\left\{m_{k}\right\} ;\left\{t_{k}\right\}\right)\right\rangle .
$$

Similar to the probability functions, it is advantageous to transform the time parameters to Laplace space

$$
\left|\hat{C}_{\mathcal{L}_{\mathrm{i}}}\left(\left\{\lambda_{k}\right\}\right)\right\rangle=\prod_{j \in \mathcal{L}_{\mathrm{i}}}\left(\int_{0}^{\infty} \mathrm{d} t_{j} e^{-\lambda_{j} t_{j}}\right)\left|C_{\mathcal{L}_{\mathrm{i}}}\left(\left\{t_{k}\right\}\right)\right\rangle
$$


which gives directly

$$
\left|\hat{C}_{\mathcal{L}}\left(\left\{\lambda_{k}\right\}\right)\right\rangle=\sum_{m_{j}} \prod_{j \in \mathcal{L}_{\mathrm{i}}}\left(\int_{-\infty}^{\infty} \mathrm{d} x_{j} x_{j}\right)\left|\hat{P}_{\mathcal{L}}\left(\left\{x_{k}\right\},\left\{m_{k}\right\} ;\left\{t_{k}\right\}\right)\right\rangle .
$$

We can apply this connection to Eq. (3.18) to get a recursion formula for the correlation functions

$$
\begin{aligned}
\left|\hat{C}_{\mathcal{L}_{\mathrm{i}}}\left(\left\{\lambda_{k}\right\}\right)\right\rangle= & \sum_{m_{j}} \prod_{j \in \mathcal{L}_{\mathrm{i}}}\left(\int_{-\infty}^{\infty} \mathrm{d} x_{j} x_{j}\right)\left|\hat{P}_{\mathcal{L}_{\mathrm{f}}}\left(\left\{x_{k}\right\},\left\{m_{k}\right\} ;\left\{\lambda_{k}\right\}\right)\right\rangle \\
= & {\left[\mathbf{I}-\hat{\Psi}_{0}\left(\Lambda_{\mathcal{L}_{\mathrm{i}}}\right) M\right]^{-1} } \\
& \times \sum_{\mathcal{L}_{\mathrm{f}}: \mathcal{L}_{\mathrm{i}} \supsetneq \mathcal{L}_{\mathrm{f}}} \sum_{m_{j}} \prod_{j \in \mathcal{L}_{\mathrm{i}}}\left(\int_{-\infty}^{\infty} \mathrm{d} x_{j} x_{j}\right) \hat{Q}_{\mathcal{L}_{\mathrm{i}} \mathcal{L}_{\mathrm{f}}}\left(\left\{x_{k}\right\},\left\{m_{k}\right\} ;\left\{\lambda_{k}\right\}\right)\left|\hat{P}_{\mathcal{L}_{\mathrm{f}}}\left(\left\{x_{k}\right\},\left\{m_{k}\right\} ;\left\{\lambda_{k}\right\}\right)\right\rangle .
\end{aligned}
$$

The operators $\hat{Q}_{\mathcal{L}_{\mathrm{i}} \mathcal{L}_{\mathrm{f}}}\left(\left\{x_{k}\right\},\left\{m_{k}\right\} ;\left\{\lambda_{k}\right\}\right)$ depend only on the $x_{k}$ and $m_{k}$ with indices $k \in \mathcal{L}_{\mathrm{i}} \backslash \mathcal{L}_{\mathrm{f}}$ while the $\left|\hat{P}_{\mathcal{L}_{\mathrm{f}}}\left(\left\{x_{k}\right\},\left\{m_{k}\right\} ;\left\{\lambda_{k}\right\}\right)\right\rangle$ depends only on the the $x_{k}$ and $m_{k}$ with indices $k \in \mathcal{L}_{\mathrm{f}}$. We can therefore define by using Eq. (3.14)

$$
\begin{aligned}
\hat{R}_{\mathcal{L}_{\mathrm{i}} \mathcal{L}_{\mathrm{f}}}\left(\left\{\lambda_{k}\right\}\right) & =\sum_{m_{j}} \prod_{j \in \mathcal{L}_{\mathrm{i}} \backslash \mathcal{L}_{\mathrm{f}}} \int_{-\infty}^{\infty} \mathrm{d} x_{j} x_{j} \hat{Q}_{\mathcal{L}_{\mathrm{i}} \mathcal{L}_{\mathrm{f}}}\left(\left\{x_{k}\right\},\left\{m_{k}\right\} ;\left\{\lambda_{k}\right\}\right) \\
& =\frac{1}{\prod_{k \in \mathcal{L}_{\mathrm{i}} \backslash \mathcal{L}_{\mathrm{f}}} \lambda_{k}} \sum_{l}|l\rangle\langle l| \int_{-\infty}^{\infty} \mathrm{d} \chi \sum_{m_{j}} \prod_{j \in \mathcal{L}_{\mathrm{i}} \backslash \mathcal{L}_{\mathrm{f}}} \int_{-\infty}^{\infty} \mathrm{d} x_{j} x_{j} \delta_{l m_{j}} \delta\left(\chi-x_{j}\right) \\
& \times \sum_{\mathcal{J}: \mathcal{L}_{\mathrm{i}} \supseteq \mathcal{J} \supseteq \mathcal{L}_{\mathrm{f}}}(-1)^{\left|\mathcal{J} \backslash \mathcal{L}_{\mathrm{f}}\right|} \hat{\Phi}\left(\chi, \Lambda_{\mathcal{J}}\right) M \\
& =\frac{1}{\prod_{k \in \mathcal{L}_{\mathrm{i}} \backslash \mathcal{L}_{\mathrm{f}}} \lambda_{k}} \int_{-\infty}^{\infty} \mathrm{d} \chi \chi^{\left|\mathcal{L}_{\mathrm{i}} \backslash \mathcal{L}_{\mathrm{f}}\right|} \sum_{\mathcal{J}: \mathcal{L}_{\mathrm{i}} \supseteq \mathcal{J} \supseteq \mathcal{L}_{\mathrm{f}}}(-1)^{\left|\mathcal{J} \backslash \mathcal{L}_{\mathrm{f}}\right| \hat{\Phi}\left(\chi, \Lambda_{\mathcal{J}}\right) M} \\
& =\frac{1}{\prod_{k \in \mathcal{L}_{\mathrm{i}} \backslash \mathcal{L}_{\mathrm{f}}} \lambda_{k}} \sum_{\mathcal{J}: \mathcal{L}_{\mathrm{i}} \supseteq \mathcal{J} \supseteq \mathcal{L}_{\mathrm{f}}}(-1)^{\left|\mathcal{J} \backslash \mathcal{L}_{\mathrm{f}}\right| \hat{\Psi}_{\left|\mathcal{L}_{\mathrm{i}} \backslash \mathcal{L}_{\mathrm{f}}\right|}\left(\Lambda_{\mathcal{J}}\right) M .}
\end{aligned}
$$

Using this in Eq. (5.7) gives

$$
\begin{aligned}
\left|\hat{C}_{\mathcal{L}_{\mathrm{i}}}\left(\left\{\lambda_{k}\right\}\right)\right\rangle & =\sum_{m_{j}} \prod_{j \in \mathcal{L}_{\mathrm{i}}} \int_{-\infty}^{\infty} \mathrm{d} x_{j} x_{j}\left|\hat{P}_{\mathcal{L}_{\mathrm{f}}}\left(\left\{x_{k}\right\},\left\{m_{k}\right\} ;\left\{t_{k}\right\}\right)\right\rangle \\
& =\left[\mathbf{I}-\hat{\Psi}_{0}\left(\Lambda_{\mathcal{L}_{\mathrm{i}}}\right) M\right]^{-1} \sum_{\mathcal{L}_{\mathrm{f}}: \mathcal{L}_{\mathrm{i}} \supsetneq \mathcal{L}_{\mathrm{f}}} \hat{R}_{\mathcal{L}_{\mathrm{i}} \mathcal{L}_{\mathrm{f}}}\left(\left\{\lambda_{k}\right\}\right)\left|\hat{C}_{\mathcal{L}_{\mathrm{f}}}\left(\left\{\lambda_{k}\right\}\right)\right\rangle .
\end{aligned}
$$

Equation (5.9) is the main result of this section.

\subsection{Examples}

As example, we obtain the Laplace transforms of the one- and two-point correlations by applying equation

and

$$
\left|\hat{C}_{1}(\lambda)\right\rangle=\frac{1}{\lambda}\left[\mathbf{I}-\hat{\Psi}_{0}(\lambda) M\right]^{-1}\left(\hat{\Psi}_{1}(0)-\hat{\Psi}_{1}(\lambda)\right)|\Sigma\rangle .
$$

$$
\begin{aligned}
\left|\hat{C}_{2}\left(\lambda_{1}, \lambda_{2}\right)\right\rangle= & {\left[\mathbf{I}-\hat{\Psi}_{0}\left(\lambda_{1}+\lambda_{2}\right) M\right]^{-1}\left(\frac{1}{\lambda_{1} \lambda_{2}}\left(\hat{\Psi}_{2}(0)-\hat{\Psi}_{2}\left(\lambda_{1}\right)-\hat{\Psi}_{2}\left(\lambda_{2}\right)+\hat{\Psi}_{2}\left(\lambda_{1}+\lambda_{2}\right)\right)|\Sigma\rangle\right.} \\
& \left.+\frac{1}{\lambda_{1}}\left(\hat{\Psi}_{1}\left(\lambda_{2}\right)-\hat{\Psi}_{1}\left(\lambda_{1}+\lambda_{2}\right)\right) M\left|\hat{C}_{1}\left(\lambda_{2}\right)\right\rangle+\frac{1}{\lambda_{2}}\left(\hat{\Psi}_{1}\left(\lambda_{1}\right)-\hat{\Psi}_{1}\left(\lambda_{1}+\lambda_{2}\right)\right) M\left|\hat{C}_{1}\left(\lambda_{1}\right)\right\rangle\right)
\end{aligned}
$$


For the single state model, these expressions become

$$
\hat{C}_{1}(\lambda)=\frac{1}{\lambda} \frac{\hat{\psi}_{1}^{\text {single }}(0)-\hat{\psi}_{1}^{\text {single }}(\lambda)}{1-\hat{\psi}_{0}^{\text {single }}(\lambda)}
$$

and

$$
\begin{aligned}
\hat{C}_{2}\left(\lambda_{1}, \lambda_{2}\right)= & \frac{1}{\lambda_{1} \lambda_{2}} \frac{\hat{\psi}_{2}^{\text {single }}(0)-\hat{\psi}_{2}^{\text {single }}\left(\lambda_{1}\right)-\hat{\psi}_{2}^{\text {single }}\left(\lambda_{2}\right)+\hat{\psi}_{2}^{\text {single }}\left(\lambda_{1}+\lambda_{2}\right)}{1-\hat{\psi}_{0}^{\text {single }}\left(\lambda_{1}+\lambda_{2}\right)} \\
& +\frac{1}{\lambda_{1} \lambda_{2}}\left(\frac{\hat{\psi}_{1}^{\text {single }}\left(\lambda_{2}\right)-\hat{\psi}_{1}^{\text {single }}\left(\lambda_{1}+\lambda_{2}\right)}{1-\hat{\psi}_{0}^{\text {single }}\left(\lambda_{1}+\lambda_{2}\right)}\right)\left(\frac{\hat{\psi}_{1}^{\text {single }}(0)-\hat{\psi}_{1}^{\text {single }}\left(\lambda_{2}\right)}{1-\hat{\psi}_{0}^{\text {single }}\left(\lambda_{2}\right)}\right) \\
& +\frac{1}{\lambda_{1} \lambda_{2}}\left(\frac{\hat{\psi}_{1}^{\text {single }}\left(\lambda_{1}\right)-\hat{\psi}_{1}^{\text {single }}\left(\lambda_{1}+\lambda_{2}\right)}{1-\hat{\psi}_{0}^{\text {single }}\left(\lambda_{1}+\lambda_{2}\right)}\right)\left(\frac{\hat{\psi}_{1}^{\text {single }}(0)-\hat{\psi}_{1}^{\text {single }}\left(\lambda_{1}\right)}{1-\hat{\psi}_{0}^{\text {single }}\left(\lambda_{1}\right)}\right) .
\end{aligned}
$$

For the alternating model, we get

$$
\begin{aligned}
\left|\hat{C}_{1}(\lambda)\right\rangle & =\frac{1}{\lambda} \frac{1}{1-\hat{\psi}^{\text {alt }}(\lambda)^{2}}\left(\begin{array}{cc}
1 & \hat{\psi}^{\text {alt }}(\lambda) \\
\hat{\psi}^{\text {alt }}(\lambda) & 1
\end{array}\right)\left(\begin{array}{cc}
I_{+}\left(1-\hat{\psi}^{\text {alt }}(\lambda)\right) & 0 \\
0 & I_{-}\left(1-\hat{\psi}^{\text {alt }}(\lambda)\right)
\end{array}\right)|\Sigma\rangle \\
& =\frac{1}{\lambda} \frac{1}{1+\hat{\psi}^{\text {alt }}(\lambda)}\left(\begin{array}{c}
I_{+}+I_{-} \hat{\psi}^{\text {alt }}(\lambda) \\
I_{+} \hat{\psi}^{\text {alt }}(\lambda)+I_{-}
\end{array}\right) \\
& =\frac{I_{+}+I_{-}}{2 \lambda}|\Sigma\rangle+\frac{I_{+}-I_{-}}{2 \lambda} \frac{1-\hat{\psi}^{\text {alt }}(\lambda)}{1+\hat{\psi}^{\text {alt }}(\lambda)}\left(\begin{array}{c}
1 \\
-1
\end{array}\right) .
\end{aligned}
$$

When we start with the equilibrium distribution, we have $\left\langle\mathrm{eq}_{\mathrm{M}} \mid \hat{C}_{1}(\lambda)\right\rangle=\left(I_{+}+I_{-}\right) /(2 \lambda)$ which is the Laplace transform of the constant function $\left\langle\mathrm{eq}_{\mathrm{M}} \mid C_{1}(t)\right\rangle=\left(I_{+}+I_{-}\right) / 2$. Hence the first term in Eq. (5.14) describes the equilibrium, while the second one describes the relaxation towards equilibrium (if we start in a non equilibrated state).

The expression for the two time correlation becomes

$$
\begin{aligned}
& \left|\hat{C}_{2}\left(\lambda_{1}, \lambda_{2}\right)\right\rangle \\
& =\frac{1}{\lambda_{1} \lambda_{2}} \frac{1}{1-\hat{\psi}^{\text {alt }}\left(\Lambda_{\{1,2\}}\right)^{2}}\left(\begin{array}{cc}
1 & \hat{\psi}^{\text {alt }}\left(\Lambda_{\{1,2\}}\right) \\
\hat{\psi}^{\text {alt }}\left(\Lambda_{\{1,2\}}\right) & 1
\end{array}\right)\left(\begin{array}{c}
I_{+}^{2}\left(1-\hat{\psi}^{\text {alt }}\left(\lambda_{1}\right)-\hat{\psi}^{\text {alt }}\left(\lambda_{2}\right)+\hat{\psi}^{\text {alt }}\left(\Lambda_{\{1,2\}}\right)\right) \\
I_{-}^{2}\left(1-\hat{\psi}^{\text {alt }}\left(\lambda_{1}\right)-\hat{\psi}^{\text {alt }}\left(\lambda_{2}\right)+\hat{\psi}^{\text {alt }}\left(\Lambda_{\{1,2\}}\right)\right)
\end{array}\right) \\
& +\frac{1}{\lambda_{1}} \frac{\hat{\psi}^{\text {alt }}\left(\lambda_{2}\right)-\hat{\psi}^{\text {alt }}\left(\Lambda_{\{1,2\}}\right)}{1-\hat{\psi}^{\text {alt }}\left(\Lambda_{\{1,2\}}\right)^{2}}\left(\begin{array}{cc}
1 & \hat{\psi}^{\text {alt }}\left(\Lambda_{\{1,2\}}\right) \\
\hat{\psi}^{\text {alt }}\left(\Lambda_{\{1,2\}}\right) & 1
\end{array}\right)\left(\begin{array}{cc}
0 & I_{+} \\
I_{-} & 0
\end{array}\right)\left|\hat{C}_{1}\left(\lambda_{2}\right)\right\rangle \\
& +\frac{1}{\lambda_{2}} \frac{\hat{\psi}^{\text {alt }}\left(\lambda_{1}\right)-\hat{\psi}^{\text {alt }}\left(\Lambda_{\{1,2\}}\right)}{1-\hat{\psi}^{\text {alt }}\left(\Lambda_{\{1,2\}}\right)^{2}}\left(\begin{array}{cc}
1 & \hat{\psi}^{\text {alt }}\left(\Lambda_{\{1,2\}}\right) \\
\hat{\psi}^{\text {alt }}\left(\Lambda_{\{1,2\}}\right) & 1
\end{array}\right)\left(\begin{array}{cc}
0 & I_{+} \\
I_{-} & 0
\end{array}\right)\left|\hat{C}_{1}\left(\lambda_{1}\right)\right\rangle \\
& =\frac{I_{+}^{2}+I_{-}^{2}}{2 \lambda_{1} \lambda_{2}}|\Sigma\rangle-\frac{\left(I_{+}-I_{-}\right)^{2}}{2 \lambda_{1} \lambda_{2}} \frac{1}{1-\hat{\psi}^{\text {alt }}\left(\Lambda_{\{1,2\}}\right)}\left(\frac{\hat{\psi}^{\text {alt }}\left(\lambda_{1}\right)-\hat{\psi}^{\text {alt }}\left(\Lambda_{\{1,2\}}\right)}{1+\hat{\psi}^{\text {alt }}\left(\lambda_{1}\right)}+\frac{\hat{\psi}^{\text {alt }}\left(\lambda_{2}\right)-\hat{\psi}^{\text {alt }}\left(\Lambda_{\{1,2\}}\right)}{1+\hat{\psi}^{\text {alt }}\left(\lambda_{2}\right)}\right)|\Sigma\rangle \\
& +\frac{I_{+}^{2}-I_{-}^{2}}{2 \lambda_{1} \lambda_{2}} \frac{1}{1+\hat{\psi}^{\text {alt }}\left(\Lambda_{\{1,2\}}\right)}\left(1-\hat{\psi}^{\text {alt }}\left(\Lambda_{\{1,2\}}\right)-\frac{\hat{\psi}^{\text {alt }}\left(\lambda_{1}\right)-\hat{\psi}^{\text {alt }}\left(\Lambda_{\{1,2\}}\right)}{1+\hat{\psi}^{\text {alt }}\left(\lambda_{1}\right)}-\frac{\hat{\psi}^{\text {alt }}\left(\lambda_{2}\right)-\hat{\psi}^{\text {alt }}\left(\Lambda_{\{1,2\}}\right)}{1+\hat{\psi}^{\text {alt }}\left(\lambda_{2}\right)}\right) \\
& \times\left(\begin{array}{c}
1 \\
-1
\end{array}\right) \text {. }
\end{aligned}
$$




\section{Correlation Functions of an Aged Process}

Similar to Section 4 we are also interested in the correlation functions of a process which has aged a time $t_{\mathrm{a}}$ before observation. In this case we are interested in the correlation functions $\left\langle X\left(t_{1}+t_{\mathrm{a}}\right) \cdots X\left(t_{n}+t_{\mathrm{a}}\right)\right\rangle_{\langle\text {init }|}$. Similar to the last section, we define

$$
\begin{aligned}
\left\langle\operatorname{init} \mid C_{\mathcal{L}}^{\text {aged }}\left(\left\{t_{k}\right\} ; t_{\mathrm{a}}\right)\right\rangle & =\left\langle\prod_{j \in \mathcal{L}} X\left(t_{j}+t_{\mathrm{a}}\right)\right\rangle_{\langle\text {init }|} \\
& =\left\langle\operatorname{init} \mid C_{\mathcal{L}}\left(\left\{t_{k}+t_{\mathrm{a}}\right\}\right)\right\rangle .
\end{aligned}
$$

We later use the limit $t_{\mathrm{a}} \rightarrow \infty$ to find the stationary correlation functions. While the aged correlations are clearly related the correlations without aging by a time transform, the form derived here is better suited for the later calculations. Similar to the multi-point correlation, often the aged multi-point correlation is treated more easily with its Laplace transform. Here, also the aging time $t_{\mathrm{a}}$ is Laplace transformed with the Laplace partner $s_{\mathrm{a}}$

$$
\left|\hat{C}_{\mathcal{L}}^{\text {aged }}\left(\left\{\lambda_{k}\right\} ; s_{\mathrm{a}}\right)\right\rangle=\int_{0}^{\infty} \mathrm{d} t_{\mathrm{a}} \exp \left(-s_{\mathrm{a}} t_{\mathrm{a}}\right) \prod_{j \in \mathcal{L}}\left(\int_{0}^{\infty} \mathrm{d} t_{j} \exp \left(-\lambda_{j} t_{j}\right)\right)\left|C_{\mathcal{L}}^{\mathrm{aged}}\left(\left\{t_{k}\right\} ; t_{\mathrm{a}}\right)\right\rangle .
$$

As in Section 5 we can get the correlation from the probability densities via

$$
\left|\hat{C}_{\mathcal{L}}^{\text {aged }}\left(\left\{\lambda_{k}\right\} ; s_{\mathrm{a}}\right)\right\rangle=\sum_{n} \int_{-\infty}^{\infty} \mathrm{d} y \sum_{m_{j}} \prod_{j \in \mathcal{L}}\left(\int_{-\infty}^{\infty} \mathrm{d} x_{j}\right)\left|\hat{P}_{\mathcal{L}}^{\text {aged }}\left(\left\{x_{k}\right\},\left\{m_{k}\right\}, y, n ;\left\{\lambda_{k}\right\}, s_{\mathrm{a}}\right)\right\rangle .
$$

In combination with Eq. (4.14) this gives a recursion formula for

$$
\left|\hat{C}_{\mathcal{L}_{\mathrm{i}}}^{\text {aged }}\left(\left\{\lambda_{k}\right\}, s_{\mathrm{a}}\right)\right\rangle=\sum_{\mathcal{L}_{\mathrm{f}}: \mathcal{L}_{\mathrm{i}} \supseteq \mathcal{L}_{\mathrm{f}}} \hat{R}_{\mathcal{L}_{\mathrm{i}} \mathcal{L}_{\mathrm{f}}}^{\mathrm{fw}}\left(\left\{\lambda_{k}\right\}, s_{\mathrm{a}}\right)\left|\hat{C}_{\mathcal{L}_{\mathrm{f}}}\left(\left\{\lambda_{k}\right\}\right)\right\rangle .
$$

with the step operator

$$
\hat{R}_{\mathcal{L}_{\mathrm{i}} \mathcal{L}_{\mathrm{f}}}^{\mathrm{fw}}\left(\left\{\lambda_{k}\right\} ; s_{\mathrm{a}}\right)=\frac{1}{\prod_{k \in \mathcal{L}_{\mathrm{i}} \backslash \mathcal{L}_{\mathrm{f}}} \lambda_{k}} \sum_{\mathcal{J}: \mathcal{L}_{\mathrm{i}} \supseteq \mathcal{J} \supseteq \mathcal{L}_{\mathrm{f}}}(-1)^{\left|\mathcal{J} \backslash \mathcal{L}_{\mathrm{f}}\right|} \hat{\Psi}_{\left|\mathcal{L}_{\mathrm{i}}^{\mathrm{fw}} \backslash \mathcal{L}_{\mathrm{f}}\right|}\left(\Lambda_{\mathcal{J}} ; s_{\mathrm{a}}\right) M .
$$

The Eqs. (6.4) and (6.5) are the main result of this section. All terms of the right hand side are know by the recursion relation Eq. (5.9).

So, the one point aged correlation is given by

$$
\left|\hat{C}_{1}^{\mathrm{aged}}\left(\lambda ; s_{\mathrm{a}}\right)\right\rangle=\hat{\Psi}_{0}^{\mathrm{fw}}\left(\lambda ; s_{\mathrm{a}}\right) M\left|\hat{C}_{1}(\lambda)\right\rangle+\frac{1}{\lambda}\left(\hat{\Psi}_{1}^{\mathrm{fw}}\left(0 ; s_{\mathrm{a}}\right)-\hat{\Psi}_{1}^{\mathrm{fw}}\left(\lambda ; s_{\mathrm{a}}\right)\right) M|\Sigma\rangle .
$$

Further simplification leads to

$$
\begin{aligned}
\left|\hat{C}_{1}^{\mathrm{aged}}\left(\lambda ; s_{\mathrm{a}}\right)\right\rangle= & {\left[\mathbf{I}-\hat{\Psi}_{0}\left(s_{\mathrm{a}}\right) M\right]^{-1} \frac{\hat{\Psi}_{0}(\lambda)-\hat{\Psi}_{0}\left(s_{\mathrm{a}}\right)}{s_{\mathrm{a}}-\lambda} M\left|\hat{C}_{1}(\lambda)\right\rangle } \\
& +\frac{1}{\lambda}\left[\mathbf{I}-\hat{\Psi}_{0}\left(s_{\mathrm{a}}\right) M\right]^{-1}\left(\frac{\hat{\Psi}_{1}(0)-\hat{\Psi}_{1}\left(s_{\mathrm{a}}\right)}{s_{\mathrm{a}}}-\frac{\hat{\Psi}_{1}(\lambda)-\hat{\Psi}_{1}\left(s_{\mathrm{a}}\right)}{s_{\mathrm{a}}-\lambda}\right) M|\Sigma\rangle \\
= & \frac{1}{s_{\mathrm{a}}-\lambda}\left|\hat{C}_{1}(\lambda)\right\rangle-\frac{1}{s_{\mathrm{a}}-\lambda}\left|\hat{C}_{1}\left(s_{\mathrm{a}}\right)\right\rangle .
\end{aligned}
$$

In this case, we could have gotten this results more directly by

$$
\begin{aligned}
\left|\hat{C}_{1}^{\text {aged }}\left(\lambda ; s_{\mathrm{a}}\right)\right\rangle & =\int_{0}^{\infty} \mathrm{d} t \int_{0}^{\infty} \mathrm{d} t_{\mathrm{a}} \exp \left(-\lambda t-s_{\mathrm{a}} t_{\mathrm{a}}\right)\left|C_{1}\left(t+t_{\mathrm{a}}\right)\right\rangle \\
& =\frac{1}{s_{\mathrm{a}}-\lambda} \int_{0}^{\infty} \mathrm{d} \tau\left(e^{-\lambda \tau}-e^{-s_{\mathrm{a}} \tau}\right)\left|C_{1}(\tau)\right\rangle \\
& =\frac{1}{s_{\mathrm{a}}-\lambda}\left|\hat{C}_{1}(\lambda)\right\rangle-\frac{1}{s_{\mathrm{a}}-\lambda}\left|\hat{C}_{1}\left(s_{\mathrm{a}}\right)\right\rangle .
\end{aligned}
$$


We also get the two point aged correlation

$$
\begin{aligned}
\left|\hat{C}_{2}^{\mathrm{aged}}\left(\lambda_{1}, \lambda_{2} ; s_{\mathrm{a}}\right)\right\rangle= & \hat{\Psi}_{0}^{\mathrm{fw}}\left(\lambda_{1}+\lambda_{2} ; s_{\mathrm{a}}\right) M\left|\hat{C}_{2}\left(\lambda_{1}, \lambda_{2}\right)\right\rangle+\frac{\hat{\Psi}_{1}^{\mathrm{fw}}\left(\lambda_{2} ; s_{\mathrm{a}}\right)-\hat{\Psi}_{1}^{\mathrm{fw}}\left(\lambda_{1}+\lambda_{2} ; s_{\mathrm{a}}\right)}{\lambda_{1}} M\left|\hat{C}_{1}\left(\lambda_{2}\right)\right\rangle \\
& +\frac{\hat{\Psi}_{1}^{\mathrm{fw}}\left(\lambda_{1} ; s_{\mathrm{a}}\right)-\hat{\Psi}_{1}^{\mathrm{fw}}\left(\lambda_{1}+\lambda_{2} ; s_{\mathrm{a}}\right)}{\lambda_{2}} M\left|\hat{C}_{1}\left(\lambda_{1}\right)\right\rangle \\
& +\frac{\hat{\Psi}_{2}^{\mathrm{fw}}\left(0 ; s_{\mathrm{a}}\right)-\hat{\Psi}_{2}^{\mathrm{fw}}\left(\lambda_{1} ; s_{\mathrm{a}}\right)-\hat{\Psi}_{2}^{\mathrm{fw}}\left(\lambda_{2} ; s_{\mathrm{a}}\right)+\hat{\Psi}_{2}^{\mathrm{fw}}\left(\lambda_{1}+\lambda_{2} ; s_{\mathrm{a}}\right)}{\lambda_{1} \lambda_{2}} M|\Sigma\rangle .
\end{aligned}
$$

\section{Stationary Behavior in Systems with Finite Mean Waiting Time}

\subsection{Stationary Forward Recurrence Time Distributions}

We have already defined the diagonal matrices $K_{n}$ describing the expansion of $\hat{\Psi}_{n}(\lambda)$ (see Eq. (2.13)). In the case of a finite mean waiting time, we have

$$
\hat{\Psi}_{0}(0)-\hat{\Psi}_{0}(\lambda)=K_{0} \lambda+\mathrm{o}(\lambda)
$$

with $\bar{\tau}=\left\langle\mathrm{eq}_{\mathrm{M}}\left|K_{0}\right| \Sigma\right\rangle$. We will also assume that

$$
\hat{\Psi}_{n}(0)-\hat{\Psi}_{n}(\lambda)=K_{n} \lambda+o(\lambda)
$$

so $K_{n}=-\hat{\Psi}_{n}^{\prime}(0)$.

Under these circumstances, we can look at the stationary behavior for long times. Formally this is described by the aged correlations for $t_{\mathrm{a}} \rightarrow \infty$ :

$$
\lim _{t_{\mathrm{a}} \rightarrow \infty}\left|C_{\mathcal{L}}^{\mathrm{aged}}\left(\left\{t_{k}\right\} ; t_{\mathrm{a}}\right)\right\rangle
$$

which is calculated in Laplace space with the final value theorem via

$$
\lim _{s_{\mathrm{a}} \rightarrow 0} s_{\mathrm{a}}\left|\hat{C}_{\mathcal{L}}^{\text {aged }}\left(\left\{\lambda_{k}\right\} ; s_{\mathrm{a}}\right)\right\rangle
$$

Central element is the asymptotic forward recurrence time described by the matrix $\hat{\Phi}^{\mathrm{fw}}\left(x, \lambda ; s_{\mathrm{a}}\right)$ (see Eq. (4.4)). Taking the limit for the stationary state in Laplace space gives the definition

$$
\begin{aligned}
\hat{\Phi}^{\mathrm{fw}, \operatorname{asym}}\left(x, \lambda_{\mathrm{f}}\right) & =\lim _{s_{\mathrm{a}} \rightarrow 0} s_{\mathrm{a}} \hat{\Phi}^{\mathrm{fw}}\left(x, \lambda_{\mathrm{f}} ; s_{\mathrm{a}}\right) \\
& =\lim _{s_{\mathrm{a}} \rightarrow 0} s_{\mathrm{a}} \frac{1}{s_{\mathrm{a}}-\lambda_{\mathrm{f}}}\left[\mathbf{I}-\hat{\Psi}_{0}\left(s_{\mathrm{a}}\right) M\right]^{-1}\left(\hat{\Phi}\left(x, \lambda_{\mathrm{f}}\right)-\hat{\Phi}\left(x, s_{\mathrm{a}}\right)\right) \\
& =\left(\lim _{s_{\mathrm{a}} \rightarrow 0} s_{\mathrm{a}}\left[\mathbf{I}-\hat{\Psi}_{0}\left(s_{\mathrm{a}}\right) M\right]^{-1}\right) \frac{1}{\lambda_{\mathrm{f}}}\left(\hat{\Phi}(x, 0)-\hat{\Phi}\left(x, \lambda_{\mathrm{f}}\right)\right) .
\end{aligned}
$$

We have to determine the behavior of $\left[\mathbf{I}-\hat{\Psi}_{0}\left(s_{\mathrm{a}}\right) M\right]^{-1}$ around $s_{\mathrm{a}} \rightarrow 0$. For $s_{\mathrm{a}}=0$ the matrix $\mathbf{I}-$ $\hat{\Psi}_{0}(0) M=\mathbf{I}-M\left(\right.$ as $\left.\hat{\Psi}_{0}(0)=\mathbf{I}\right)$ has a single eigenvalue 0 with right eigenvector $|\Sigma\rangle$ and left eigenvector $\langle\mathrm{eq}|$

$$
\begin{aligned}
\left(\mathbf{I}-\hat{\Psi}_{0}(0) M\right)|\Sigma\rangle & =0 \\
\text { and } \quad\left\langle\mathrm{eq}_{\mathrm{M}}\right|\left(\mathbf{I}-\hat{\Psi}_{0}(0) M\right) & =0,
\end{aligned}
$$


which follows from probability conservation $(M|\Sigma\rangle=|\Sigma\rangle)$ and the ergodicity of the Markov chain $\left(\left\langle\mathrm{eq}_{\mathrm{M}}\right| M=\left\langle\mathrm{eq}_{\mathrm{M}}\right|\right)$. The dominant behavior of $\left[\mathbf{I}-\hat{\Psi}_{0}\left(s_{\mathrm{a}}\right) M\right]^{-1}$ for small $s$ is determined by this eigenvector and eigenvalue. We will employ here the physical techniques of stationary perturbation theory, more mathematical rigorous approaches are described in [24]. We consider the perturbation

$$
\mathbf{I}-\hat{\Psi}_{0}\left(s_{\mathrm{a}}\right) M=\mathbf{I}-M+s_{\mathrm{a}} K_{0} M+\mathrm{o}\left(s_{\mathrm{a}}\right) .
$$

The eigenvalue changes in first order (using Eq. (2.12))

$$
\begin{aligned}
\left\langle\mathrm{eq}_{\mathrm{M}}\left|\mathbf{I}-M+s_{\mathrm{a}} K_{0} M\right| \Sigma\right\rangle+\mathrm{o}\left(s_{\mathrm{a}}\right) & =s_{\mathrm{a}}\left\langle\mathrm{eq}_{\mathrm{M}}\left|K_{0}\right| \Sigma\right\rangle+\mathrm{o}\left(s_{\mathrm{a}}\right) \\
& =s_{\mathrm{a}} \bar{\tau}+\mathrm{o}\left(s_{\mathrm{a}}\right) .
\end{aligned}
$$

As the changes in the right and left eigenvalue are of order $\mathrm{O}\left(s_{\mathrm{a}}\right)$ and the projection on the eigenspace (to $\left.s_{\mathrm{a}}=0\right)$ is given by

$$
|\Sigma\rangle\left\langle\mathrm{eq}_{\mathrm{M}}\right|
$$

we have

$$
\begin{aligned}
{\left[\mathbf{I}-\hat{\Psi}_{0}\left(s_{\mathrm{a}}\right) M\right]^{-1} } & =\frac{|\Sigma\rangle\left\langle\mathrm{eq}_{\mathrm{M}}\right|+\mathrm{O}\left(s_{\mathrm{a}}\right)}{s_{\mathrm{a}} \bar{\tau}+\mathrm{o}\left(s_{\mathrm{a}}\right)} \\
& =\frac{|\Sigma\rangle\left\langle\mathrm{eq}_{\mathrm{M}}\right|}{s_{\mathrm{a}} \bar{\tau}}+\mathrm{o}\left(\frac{1}{s_{\mathrm{a}}}\right)
\end{aligned}
$$

and

$$
\lim _{s_{\mathrm{a}} \rightarrow 0} s_{\mathrm{a}}\left[\mathbf{I}-\hat{\Psi}_{0}\left(s_{\mathrm{a}}\right) M\right]^{-1}=\frac{|\Sigma\rangle\left\langle\mathrm{eq}_{\mathrm{M}}\right|}{\bar{\tau}} .
$$

This gives

$$
\hat{\Phi}^{\mathrm{fw}, \operatorname{asym}}\left(x, \lambda_{\mathrm{f}}\right)=|\Sigma\rangle \frac{1}{\bar{\tau} \lambda_{\mathrm{f}}}\left\langle\mathrm{eq}_{\mathrm{M}}\right|\left(\hat{\Phi}(x, 0)-\hat{\Phi}\left(x, \lambda_{\mathrm{f}}\right)\right) .
$$

As expected, it is independent of the initial distribution as for every distribution of initial conditions $\langle$ init|

$$
\langle\operatorname{init}| \hat{\Phi}^{\mathrm{fw}, \operatorname{asym}}\left(x, \lambda_{\mathrm{f}}\right)=\frac{1}{\bar{\tau} \lambda_{\mathrm{f}}}\left\langle\mathrm{eq}_{\mathrm{M}}\right|\left(\hat{\Phi}(x, 0)-\hat{\Phi}\left(x, \lambda_{\mathrm{f}}\right)\right) .
$$

The $x$ moments are (Eq. (4.11))

$$
\begin{aligned}
\hat{\Psi}_{n}^{\mathrm{fw}}\left(\lambda_{\mathrm{f}} ; s_{\mathrm{a}}\right) & =\int \mathrm{d} x x^{n} \hat{\Phi}^{\mathrm{fw}}\left(x, \lambda_{\mathrm{f}} ; s_{\mathrm{a}}\right) \\
& =\frac{1}{s_{\mathrm{a}}-\lambda_{\mathrm{f}}}\left[\mathbf{I}-\hat{\Psi}_{0}\left(s_{\mathrm{a}}\right) M\right]^{-1}\left(\hat{\Psi}_{n}\left(\lambda_{\mathrm{f}}\right)-\hat{\Psi}_{n}\left(s_{\mathrm{a}}\right)\right)
\end{aligned}
$$

which becomes in the asymptotic case

$$
\begin{aligned}
& \qquad \hat{\Psi}_{n}^{\mathrm{fw}, \operatorname{asym}}\left(\lambda_{\mathrm{f}}\right)=|\Sigma\rangle\left\langle\mathrm{eq}_{\mathrm{M}}\right| \frac{\hat{\Psi}_{n}(0)-\hat{\Psi}_{n}\left(\lambda_{\mathrm{f}}\right)}{\bar{\tau} \lambda_{\mathrm{f}}} \\
& \text { with special value } \hat{\Psi}_{n}^{\mathrm{fw}, \operatorname{asym}}(0)=|\Sigma\rangle\left\langle\mathrm{eq}_{\mathrm{M}}\right| \frac{K_{n}}{\bar{\tau}} .
\end{aligned}
$$

For $n=0$ we have with $\bar{\tau}=\left\langle\mathrm{eq}_{\mathrm{M}}\left|K_{0}\right| \Sigma\right\rangle$

$$
\hat{\Psi}_{0}^{\mathrm{fw}, \operatorname{asym}}(0)|\Sigma\rangle=|\Sigma\rangle
$$

which is expected by conservation of probability. Therefore the asymptotic correlation can be inferred from the aged correlations by substituting $\hat{\Psi}_{n}^{\mathrm{fw}, \text { asym }}(\lambda)$ for $\hat{\Psi}_{n}^{\mathrm{fw}}\left(\lambda ; s_{\mathrm{a}}\right)$ in Eq. (6.4) which then becomes independent of the initial distribution. 
For the single state process and $n=0$ we get

$$
\hat{\Psi}_{0}^{\mathrm{fw}, \text { asym }}\left(\lambda_{\mathrm{f}}\right)=\frac{1-\hat{\psi}_{0}^{\text {single }}\left(\lambda_{\mathrm{f}}\right)}{\bar{\tau} \lambda_{\mathrm{f}}}
$$

which is well known (e.g., [20]).

\subsection{Asymptotic Correlation}

Especially interesting is the stationary correlation function $C^{\text {cor }}(t)$

$$
C^{\text {cor }}(t)=\lim _{t_{\mathrm{a}} \rightarrow \infty}\left\langle X\left(t_{\mathrm{a}}\right) X\left(t_{\mathrm{a}}+t\right)\right\rangle_{\langle\text {init }|}
$$

and its Laplace transform

$$
\hat{C}^{\mathrm{cor}}(\lambda)=\int_{0}^{\infty} \mathrm{d} t e^{-\lambda t} C^{\mathrm{cor}}(t)
$$

From Eq. (4.2) we can determine the above correlation with

$$
\left\langle X\left(t_{\mathrm{a}}\right) X\left(t_{\mathrm{a}}+t\right)\right\rangle_{\langle\text {init }|}=\sum_{m} \sum_{n} \int_{-\infty}^{\infty} \mathrm{d} x \int_{-\infty}^{\infty} \mathrm{d} y x y\left\langle\text { init } \mid P_{1}^{\text {aged }}\left(x, m, y, n ; t, t_{\mathrm{a}}\right)\right\rangle,
$$

its double Laplace transform is

$\int_{0}^{\infty} \mathrm{d} t_{\mathrm{a}} \int_{0}^{\infty} \mathrm{d} t \exp \left(-s_{\mathrm{a}} t_{\mathrm{a}}-\lambda t\right)\left\langle X\left(t_{\mathrm{a}}\right) X\left(t_{\mathrm{a}}+t\right)\right\rangle_{\langle\text {init }|}=\sum_{m} \sum_{n} \int_{-\infty}^{\infty} \mathrm{d} x \int_{-\infty}^{\infty} \mathrm{d} y x y\left\langle\operatorname{init} \mid \hat{P}_{1}^{\text {aged }}\left(x, m, y, n ; \lambda, s_{\mathrm{a}}\right)\right\rangle$

and the limit $t_{\mathrm{a}} \rightarrow \infty$ can be done in Laplace space by the final value theorem

$$
\hat{C}^{\mathrm{cor}}(\lambda)=\lim _{s_{\mathrm{a}} \rightarrow 0} s_{\mathrm{a}} \sum_{m} \sum_{n} \int_{-\infty}^{\infty} \mathrm{d} x \int_{-\infty}^{\infty} \mathrm{d} y x y\left\langle\text { init } \mid \hat{P}_{1}^{\text {aged }}\left(x, m, y, n ; \lambda, s_{\mathrm{a}}\right)\right\rangle .
$$

The expression for $\left|\hat{P}_{1}^{\text {aged }}\left(x, m, y, n ; \lambda, s_{\mathrm{a}}\right)\right\rangle$ has been determined in Eq. (4.15). We get from this

$$
\begin{aligned}
& \sum_{m} \sum_{n} \int_{-\infty}^{\infty} \mathrm{d} x \int_{-\infty}^{\infty} \mathrm{d} y x y\left\langle\operatorname{init} \mid \hat{P}_{1}^{\mathrm{aged}}\left(x, m, y, n ; \lambda, s_{\mathrm{a}}\right)\right\rangle \\
& \quad=\frac{1}{\lambda}\left\langle\operatorname{init}\left|\hat{\Psi}_{1}^{\mathrm{fw}}\left(\lambda ; s_{\mathrm{a}}\right) M\left[\mathbf{I}-\hat{\Psi}_{0}(\lambda) M\right]^{-1}\left(\hat{\Psi}_{1}(0)-\hat{\Psi}_{1}(\lambda)\right)\right| \Sigma\right\rangle+\frac{1}{\lambda}\left\langle\operatorname{init}\left|\left(\hat{\Psi}_{2}^{\mathrm{fw}}\left(0 ; s_{\mathrm{a}}\right)-\hat{\Psi}_{2}^{\mathrm{fw}}\left(\lambda ; s_{\mathrm{a}}\right)\right)\right| \Sigma\right\rangle
\end{aligned}
$$

and (using Eq. (7.16))

$$
\begin{aligned}
\hat{C}^{\mathrm{cor}}(\lambda)= & \lim _{s_{\mathrm{a}} \rightarrow 0} s_{\mathrm{a}}\left(\frac{1}{\lambda}\left\langle\operatorname{init}\left|\hat{\Psi}_{1}^{\mathrm{fw}}\left(\lambda ; s_{\mathrm{a}}\right) M\left[\mathbf{I}-\hat{\Psi}_{0}(\lambda) M\right]^{-1}\left(\hat{\Psi}_{1}(0)-\hat{\Psi}_{1}(\lambda)\right)\right| \Sigma\right\rangle\right. \\
& \left.+\frac{1}{\lambda}\left\langle\operatorname{init}\left|\left(\hat{\Psi}_{2}^{\mathrm{fw}}\left(0 ; s_{\mathrm{a}}\right)-\hat{\Psi}_{2}^{\mathrm{fw}}\left(\lambda ; s_{\mathrm{a}}\right)\right)\right| \Sigma\right\rangle\right) \\
= & \frac{1}{\lambda}\left\langle\operatorname{init}\left|\hat{\Psi}_{1}^{\mathrm{fw}, \operatorname{asym}}\left(\lambda ; s_{\mathrm{a}}\right) M\left[\mathbf{I}-\hat{\Psi}_{0}(\lambda) M\right]^{-1}\left(\hat{\Psi}_{1}(0)-\hat{\Psi}_{1}(\lambda)\right)\right| \Sigma\right\rangle \\
& +\frac{1}{\lambda}\left\langle\operatorname{init}\left|\left(\hat{\Psi}_{2}^{\mathrm{fw}, \operatorname{asym}}\left(0 ; s_{\mathrm{a}}\right)-\hat{\Psi}_{2}^{\mathrm{fw}, \operatorname{asym}}\left(\lambda ; s_{\mathrm{a}}\right)\right)\right| \Sigma\right\rangle \\
= & \frac{1}{\bar{\tau} \lambda^{2}}\left\langle\operatorname{eq} \mathrm{M}\left|\left(\hat{\Psi}_{1}(0)-\hat{\Psi}_{1}(\lambda)\right) M\left[\mathbf{I}-\hat{\Psi}_{0}(\lambda) M\right]^{-1}\left(\hat{\Psi}_{1}(0)-\hat{\Psi}_{1}(\lambda)\right)\right| \Sigma\right\rangle \\
& -\frac{1}{\bar{\tau} \lambda^{2}}\left\langle\operatorname{eq}_{\mathrm{M}}\left|\hat{\Psi}_{2}(0)-K_{2} \lambda-\hat{\Psi}_{2}(\lambda)\right| \Sigma\right\rangle .
\end{aligned}
$$


Writing it completely in terms of the $\hat{\Psi}_{n}(\lambda)$

$$
\begin{aligned}
\hat{C}^{\mathrm{cor}}(\lambda)= & -\frac{1}{\lambda^{2}} \frac{1}{\left\langle\mathrm{eq}_{\mathrm{M}}\left|\hat{\Psi}_{0}^{\prime}(0)\right| \Sigma\right\rangle}\left\langle\mathrm{eq}_{\mathrm{M}}\left|\left(\hat{\Psi}_{1}(0)-\hat{\Psi}_{1}(\lambda)\right) M\left[\mathbf{I}-\hat{\Psi}_{0}(\lambda) M\right]^{-1}\left(\hat{\Psi}_{1}(0)-\hat{\Psi}_{1}(\lambda)\right)\right| \Sigma\right\rangle \\
& +\frac{1}{\lambda^{2}} \frac{1}{\left\langle\mathrm{eq}_{\mathrm{M}}\left|\hat{\Psi}_{0}^{\prime}(0)\right| \Sigma\right\rangle}\left\langle\mathrm{eq}_{\mathrm{M}}\left|\hat{\Psi}_{2}(0)+\hat{\Psi}_{2}^{\prime}(0) \lambda-\hat{\Psi}_{2}(\lambda)\right| \Sigma\right\rangle .
\end{aligned}
$$

\subsection{Power Spectral Density}

The power spectral density $\bar{S}(\omega)$ of this process can be determined by using the Wiener-Khinchin theorem

$$
\bar{S}(\omega)=\int_{-\infty}^{\infty} \mathrm{d} t e^{i \omega t} C^{\mathrm{cor}}(t)
$$

which can be written in terms of the $\hat{C}^{\mathrm{cor}}(\lambda)$ :

$$
\begin{aligned}
\bar{S}(\omega) & =\int_{-\infty}^{\infty} \mathrm{d} t e^{i \omega t} C^{\mathrm{cor}}(t) \\
& =\hat{C}^{\mathrm{cor}}(i \omega)+\hat{C}^{\mathrm{cor}}(-i \omega) .
\end{aligned}
$$

Hence, with Eq. (7.25) we have a formula for the power spectrum of a stationary process.

In general, if the correlation for large $t$ behaves as

$$
C^{\mathrm{cor}}(t) \simeq \frac{D}{t^{\gamma}}
$$

with $0<\gamma<1$, its Laplace transform behaves for small $\lambda$ as (Tauberian theorems)

$$
\hat{C}^{\mathrm{cor}}(\lambda) \simeq \Gamma(1-\gamma) D \lambda^{\gamma-1}
$$

which results in the low frequency behavior

$$
\begin{aligned}
\bar{S}(\omega) & =\Gamma(1-\gamma) D\left((i \omega)^{\gamma-1}+(-i \omega)^{\gamma-1}\right)+\mathrm{o}\left(\omega^{\gamma-1}\right) \\
& =2 \sin (\gamma \pi / 2) \Gamma(1-\gamma) D \frac{1}{\omega^{1-\gamma}}+\mathrm{o}\left(\omega^{\gamma-1}\right) .
\end{aligned}
$$

For our model, we get (using Eq. (7.25)) for $\omega \neq 0$

$$
\begin{aligned}
\bar{S}(\omega)=\hat{C}^{\mathrm{cor}}(i \omega)+\hat{C}^{\mathrm{cor}}(-i \omega) & \\
=\frac{1}{\omega^{2}} \frac{1}{\left\langle\mathrm{eq}_{\mathrm{M}}\left|\hat{\Psi}_{0}^{\prime}(0)\right| \Sigma\right\rangle}( & \left\langle\mathrm{eq}_{\mathrm{M}}\left|\left(\hat{\Psi}_{1}(0)-\hat{\Psi}_{1}(i \omega)\right) M\left[\mathbf{I}-\hat{\Psi}_{0}(i \omega) M\right]^{-1}\left(\hat{\Psi}_{1}(0)-\hat{\Psi}_{1}(i \omega)\right)\right| \Sigma\right\rangle \\
& +\left\langle\mathrm{eq}_{\mathrm{M}}\left|\left(\hat{\Psi}_{1}(0)-\hat{\Psi}_{1}(-i \omega)\right) M\left[\mathbf{I}-\hat{\Psi}_{0}(-i \omega) M\right]^{-1}\left(\hat{\Psi}_{1}(0)-\hat{\Psi}_{1}(-i \omega)\right)\right| \Sigma\right\rangle \\
& \left.-\left\langle\mathrm{eq}_{\mathrm{M}}\left|2 \hat{\Psi}_{2}(0)-\hat{\Psi}_{2}(i \omega)-\hat{\Psi}_{2}(-i \omega)\right| \Sigma\right\rangle\right) .
\end{aligned}
$$

The condition $\omega \neq 0$ is needed to ensure that $\mathbf{I}-\hat{\Psi}_{0}(i \omega) M$ is invertible.

In this case we can also look at the low-frequency behavior. We will assume that the second moment of the average waiting time distribution $\left\langle\mathrm{eq}_{\mathrm{M}}\left|\Psi_{0}(t)\right| \Sigma\right\rangle$ is infinite. More specifically, we will assume that we have an expansion

$$
\hat{\Psi}_{n}(\lambda)=\hat{\Psi}_{n}(0)-\lambda K_{n}+\lambda^{1+\beta} L_{n}+\mathrm{o}\left(\lambda^{1+\beta}\right)
$$


with $0<\beta<1$. In principal it is possible to have a different $\beta$ for the different $n$ moments, but we will only consider here this simpler case.

The calculation are very similar to the one performed above, but also very technical. Therefore they are presented in App. B. The result is Eq. (B.5)

$\bar{S}(\omega)=\left[\frac{\left\langle\mathrm{eq}_{\mathrm{M}}\left|L_{2}\right| \Sigma\right\rangle}{\left\langle\mathrm{eq}_{\mathrm{M}}\left|L_{0}\right| \Sigma\right\rangle}-2 \frac{\left\langle\mathrm{eq}_{\mathrm{M}}\left|L_{1}\right| \Sigma\right\rangle\left\langle\mathrm{eq}_{\mathrm{M}}\left|K_{1}\right| \Sigma\right\rangle}{\left\langle\mathrm{eq}_{\mathrm{M}}\left|L_{0}\right| \Sigma\right\rangle\left\langle\mathrm{eq}_{\mathrm{M}}\left|K_{0}\right| \Sigma\right\rangle}+\frac{\left\langle\mathrm{eq}_{\mathrm{M}}\left|K_{1}\right| \Sigma\right\rangle^{2}}{\left\langle\mathrm{eq}_{\mathrm{M}}\left|K_{0}\right| \Sigma\right\rangle^{2}}\right] \frac{\left\langle\mathrm{eq}_{\mathrm{M}}\left|L_{0}\right| \Sigma\right\rangle}{\left\langle\mathrm{eq}_{\mathrm{M}}\left|K_{0}\right| \Sigma\right\rangle} \frac{2 \sin \left(\beta \frac{\pi}{2}\right)}{\omega^{1-\beta}}+\mathrm{o}\left(\omega^{\beta-1}\right)$

We have a $1 / f$ noise. This expression can be simplified by using the second moment of the long time distribution centered to the mean value of the process (Eq. (C.19), using the definitions Eqs. (2.14), (2.16) and $(2.17))$

$$
\begin{aligned}
\operatorname{Var}^{\text {center }} & =\int_{-\infty}^{\infty} \mathrm{d} x\left(x-\mu_{1}^{\mathrm{asym}}\right)^{2} \bar{\phi}^{\mathrm{lt}}(x) \\
& =\left(\bar{\psi}_{1}^{\mathrm{lt}}-\mu_{1}^{\mathrm{asym}}\right)^{2}+\sigma_{\mathrm{lt}}^{2}
\end{aligned}
$$

which gives

$$
\bar{S}(\omega)=\operatorname{Var}^{\text {center }} \frac{\left\langle\mathrm{eq}_{\mathrm{M}}\left|L_{0}\right| \Sigma\right\rangle}{\left\langle\mathrm{eq}_{\mathrm{M}}\left|K_{0}\right| \Sigma\right\rangle} \frac{2 \sin \left(\beta \frac{\pi}{2}\right)}{\omega^{1-\beta}}+\mathrm{o}\left(\omega^{\beta-1}\right)
$$

\section{The Expected Value of the Spectrum}

\subsection{Determination of the Spectrum}

In the last Sec. 7 we have determined the spectral density by means of the Wiener-Khinchin theorem. This works only for stationary processes (in the weak sense). But as soon the mean waiting time is infinite, the processes considered here, do not fulfill this requirement. Additionally, when working with data (experimental or simulated), one usually does not have the correlation function, but has to estimate the power spectrum from the data. Many methods have been established to accomplish this task [41]. A central element is the so called periodogram on which properties many other methods of spectral estimation rely.

For the stochastic process $X(t)$ we define its Fourier transform up to time $T$

$$
F_{T}(\omega)=\int_{0}^{T} \mathrm{~d} t \exp (i \omega t) X(t)
$$

The spectrum determined from the interval $[0, T]$ is given by

$$
S_{T}(\omega)=\frac{1}{T} F_{T}(-\omega) F_{T}(\omega)
$$

which is the definition of the periodogram. In the case of stationary processes the expected value of the periodogram converges towards to spectral density

$$
\bar{S}(\omega)=\lim _{T \rightarrow \infty}\left\langle S_{T}(\omega)\right\rangle_{\langle\text {init }|} .
$$

A problem of the periodogram is that it is not a consistent estimator of the spectrum, i.e., its variance will not decrease when the observation time goes to infinity. This is one of the reasons why many methods try to introduce an averaging to reduce the variance. Nevertheless, many properties of other estimators can be deduced from the understanding of the periodogram. 
We also focus for the most time on the non-stationary case. We assume that we have the following expansion of the waiting times for small Laplace parameters $\lambda$

$$
\begin{aligned}
\hat{\Psi}_{0}(\lambda) & =\hat{\Psi}_{0}(0)-\lambda^{\alpha} K_{0}+\mathrm{o}\left(\lambda^{\alpha}\right) \\
& =\mathbf{I}-\lambda^{\alpha} K_{0}+\mathrm{o}\left(\lambda^{\alpha}\right)
\end{aligned}
$$

with $0<\alpha \leq 1$. Here $\alpha=1$ is the stationary case. The value $\alpha$ has been introduced in Section 2 and $\bar{\tau}^{\alpha}=\left\langle\mathrm{eq}_{\mathrm{M}}\left|K_{0}\right| \Sigma\right\rangle($ Eq. $(2.12))$.

We are interested in this section in the behavior of $\left\langle S_{T}(\omega)\right\rangle$ for large $T$. We will find, that the limit $\lim _{T \rightarrow \infty}\left\langle S_{T}(\omega)\right\rangle=0$ for $\alpha<1$. Instead, the value of $\left\langle S_{T}(\omega)\right\rangle$ decays with $T^{\alpha-1}$ (as shown later) which motivates the following definition

$$
\bar{S}_{\alpha}(\omega)=\lim _{T \rightarrow \infty} T^{1-\alpha}\left\langle S_{T}(\omega)\right\rangle_{\langle\text {init }|} .
$$

The formalism using the multi-point correlations can be used by a little trick of introducing several times

$$
T\left\langle S_{T}(\omega)\right\rangle_{\langle\text {init }|}=\left.\left\langle F_{T_{1}}(-\omega) F_{T_{2}}(\omega)\right\rangle_{\langle\text {init }|}\right|_{T_{1}=T_{2}=T} .
$$

We have to determine $\left\langle F_{T_{1}}(-\omega) F_{T_{2}}(\omega)\right\rangle_{\langle\text {init }|}$ for large $T_{1}$ and $T_{2}$ where the ratio of $T_{1}$ and $T_{2}$ stays constant. This is easily done by introducing a scaling factor $\zeta$

$$
\zeta^{\alpha}\left\langle F_{T_{1} / \zeta}(-\omega) F_{T_{2} / \zeta}(\omega)\right\rangle_{\langle\text {init }|}
$$

and letting $\zeta \rightarrow 0$. The scaling $\zeta^{\alpha}$ corresponds to the large $T$ behavior $T\left\langle S_{T}(\omega)\right\rangle \sim T^{-\alpha}$. Taking the Laplace transform with the Laplace pairs $\lambda_{1} \leftrightarrow T_{1}, \lambda_{2} \leftrightarrow T_{2}$

$$
\begin{aligned}
& \mathrm{L}\left[\zeta^{\alpha}\left\langle F_{T_{1} / \zeta}(-\omega) F_{T_{2} / \zeta}(\omega)\right\rangle_{\langle\text {init }|}\right] \\
& =\zeta^{\alpha} \int_{0}^{\infty} \mathrm{d} T_{1} \int_{0}^{\infty} \mathrm{d} T_{2} e^{-\lambda_{1} T_{1}-\lambda_{2} T_{2}} \int_{0}^{T_{1} / \zeta} \mathrm{d} t_{1} \int_{0}^{T_{2} / \zeta} \mathrm{d} t_{2} e^{i \omega\left(t_{2}-t_{1}\right)}\left\langle X\left(t_{1}\right) X\left(t_{2}\right)\right\rangle_{\langle\text {init }|} \\
& =\frac{\zeta^{\alpha}}{\lambda_{1} \lambda_{2}} \int_{0}^{\infty} \mathrm{d} T_{1} e^{-\left(\zeta \lambda_{1}+i \omega\right) T_{1}} \int_{0}^{\infty} \mathrm{d} T_{2} e^{-\left(\zeta \lambda_{2}-i \omega\right) T_{2}}\left\langle X\left(T_{1}\right) X\left(T_{2}\right)\right\rangle_{\langle\text {init }|} \\
& =\frac{\zeta^{\alpha}}{\lambda_{1} \lambda_{2}}\left\langle\text { init } \mid \hat{C}_{2}\left(\zeta \lambda_{1}+i \omega, \zeta \lambda_{2}-i \omega\right)\right\rangle .
\end{aligned}
$$

Using (5.10) and (5.11) we get

$$
\begin{aligned}
& \left|\hat{C}_{2}\left(\zeta \lambda_{1}+i \omega, \zeta \lambda_{2}-i \omega\right)\right\rangle \\
& =\left[\mathbf{I}-\hat{\Psi}_{0}\left(\zeta\left(\lambda_{1}+\lambda_{2}\right)\right) M\right]^{-1} \\
& \times\left(\frac{1}{\left(\zeta \lambda_{1}+i \omega\right)\left(\zeta \lambda_{2}-i \omega\right)}\left(\hat{\Psi}_{2}(0)-\hat{\Psi}_{2}\left(\zeta \lambda_{1}+i \omega\right)-\hat{\Psi}_{2}\left(\zeta \lambda_{2}-i \omega\right)+\hat{\Psi}_{2}\left(\zeta\left(\lambda_{1}+\lambda_{2}\right)\right)\right)|\Sigma\rangle\right. \\
& +\frac{1}{\zeta \lambda_{1}+i \omega}\left(\hat{\Psi}_{1}\left(\zeta \lambda_{2}-i \omega\right)-\hat{\Psi}_{1}\left(\zeta\left(\lambda_{1}+\lambda_{2}\right)\right)\right) M\left|\hat{C}_{1}\left(\zeta \lambda_{2}-i \omega\right)\right\rangle \\
& \left.+\frac{1}{\zeta \lambda_{2}-i \omega}\left(\hat{\Psi}_{1}\left(\zeta \lambda_{1}+i \omega\right)-\hat{\Psi}_{1}\left(\zeta\left(\lambda_{1}+\lambda_{2}\right)\right)\right) M\left|\hat{C}_{1}\left(\zeta \lambda_{1}+i \omega\right)\right\rangle\right) .
\end{aligned}
$$

Analogous to Eq. (7.11) we get

$$
\begin{aligned}
{\left[\mathbf{I}-\hat{\Psi}_{0}\left(\zeta\left(\lambda_{1}+\lambda_{2}\right)\right) M\right]^{-1} } & =\frac{|\Sigma\rangle\left\langle\mathrm{eq}_{\mathrm{M}}\right|}{\left\langle\mathrm{eq}_{\mathrm{M}}\left|K_{0}\right| \Sigma\right\rangle \zeta^{\alpha}\left(\lambda_{1}+\lambda_{2}\right)^{\alpha}}+\mathrm{o}\left(\zeta^{-\alpha}\right) \\
& =\frac{|\Sigma\rangle\left\langle\mathrm{eq}_{\mathrm{M}}\right|}{\bar{\tau}^{\alpha} \zeta^{\alpha}\left(\lambda_{1}+\lambda_{2}\right)^{\alpha}}+\mathrm{o}\left(\zeta^{-\alpha}\right) .
\end{aligned}
$$


Using this result, we get in leading order in $\zeta$

$$
\begin{aligned}
& \left|\hat{C}_{2}\left(\zeta \lambda_{1}+i \omega, \zeta \lambda_{2}-i \omega\right)\right\rangle \simeq \\
& |\Sigma\rangle \frac{1}{\bar{\tau}^{\alpha} \zeta^{\alpha}\left(\lambda_{1}+\lambda_{2}\right)^{\alpha}}\left(\frac{1}{\omega^{2}}\left\langle\mathrm{eq}_{\mathrm{M}}\left|2 \hat{\Psi}_{2}(0)-\hat{\Psi}_{2}(i \omega)-\hat{\Psi}_{2}(-i \omega)\right| \Sigma\right\rangle\right. \\
& \left.+\frac{1}{i \omega}\left\langle\mathrm{eq}_{\mathrm{M}}\left|\left(\hat{\Psi}_{1}(-i \omega)-\hat{\Psi}_{1}(0)\right) M\right| \hat{C}_{1}(-i \omega)\right\rangle+\frac{1}{-i \omega}\left\langle\mathrm{eq}_{\mathrm{M}}\left|\left(\hat{\Psi}_{1}(i \omega)-\hat{\Psi}_{1}(0)\right) M\right| \hat{C}_{1}(i \omega)\right\rangle\right) \\
= & |\Sigma\rangle \frac{1}{\bar{\tau}^{\alpha} \zeta^{\alpha}\left(\lambda_{1}+\lambda_{2}\right)^{\alpha}} \frac{1}{\omega^{2}}\left(\left\langle\mathrm{eq}_{\mathrm{M}}\left|2 \hat{\Psi}_{2}(0)-\hat{\Psi}_{2}(i \omega)-\hat{\Psi}_{2}(-i \omega)\right| \Sigma\right\rangle\right. \\
& -\left\langle\mathrm{eq}_{\mathrm{M}}\left|\left(\hat{\Psi}_{1}(0)-\hat{\Psi}_{1}(i \omega)\right) M\left[\mathbf{I}-\hat{\Psi}_{0}(i \omega) M\right]^{-1}\left(\hat{\Psi}_{1}(0)-\hat{\Psi}_{1}(i \omega)\right)\right| \Sigma\right\rangle \\
& \left.-\left\langle\mathrm{eq}_{\mathrm{M}}\left|\left(\hat{\Psi}_{1}(0)-\hat{\Psi}_{1}(-i \omega)\right) M\left[\mathbf{I}-\hat{\Psi}_{0}(-i \omega) M\right]^{-1}\left(\hat{\Psi}_{1}(0)-\hat{\Psi}_{1}(-i \omega)\right)\right| \Sigma\right\rangle\right) \\
= & |\Sigma\rangle \frac{1}{\zeta^{\alpha}\left(\lambda_{1}+\lambda_{2}\right)^{\alpha}} \sigma(\omega)
\end{aligned}
$$

where the last line defines the function $\sigma(\omega)$ which collects the all $\omega$-dependent terms. We are interested in

$$
\lim _{\zeta \rightarrow 0} L\left[\zeta^{\alpha}\left\langle F_{T_{1} / \zeta}(-\omega) F_{T_{2} / \zeta}(\omega)\right\rangle_{\langle\text {init }|}\right]=\frac{1}{\lambda_{1} \lambda_{2}} \frac{1}{\left(\lambda_{1}+\lambda_{2}\right)^{\alpha}} \sigma(\omega)
$$

where we have to invert the Laplace transform for $T_{1}=T_{2}=T$. The technical details are laid out in Appendix A. We use Eq. (A.10)

$$
\mathrm{L}^{-1}\left[\frac{1}{\lambda_{1} \lambda_{2}} \frac{1}{\left(\lambda_{1}+\lambda_{2}\right)^{\alpha}} \sigma(\omega)\right]\left(T_{1}=T_{2}=T\right)=\frac{\sigma(\omega)}{\Gamma(1+\alpha)} T^{\alpha}
$$

we get for large $T$ with Eq. (8.6)

$$
\left\langle S_{T}(\omega)\right\rangle \simeq T^{\alpha-1} \frac{\sigma(\omega)}{\Gamma(1+\alpha)}
$$

Here, one sees directly, that the observed spectrum decays in the $\alpha<1$ case with the measurement time $T$ as $T^{\alpha-1}$.

In our extended definition of the expected spectrum Eq. (8.5) we have then

$$
\begin{aligned}
\bar{S}_{\alpha}(\omega)= & \frac{\sigma(\omega)}{\Gamma(1+\alpha)} \\
= & \frac{1}{\Gamma(1+\alpha)\left\langle\mathrm{eq}_{\mathrm{M}}\left|K_{0}\right| \Sigma\right\rangle} \frac{1}{\omega^{2}}\left(\left\langle\mathrm{eq}_{\mathrm{M}}\left|2 \hat{\Psi}_{2}(0)-\hat{\Psi}_{2}(i \omega)-\hat{\Psi}_{2}(-i \omega)\right| \Sigma\right\rangle\right. \\
& -\left\langle\mathrm{eq}_{\mathrm{M}}\left|\left(\hat{\Psi}_{1}(0)-\hat{\Psi}_{1}(i \omega)\right) M\left[\mathbf{I}-\hat{\Psi}_{0}(i \omega) M\right]^{-1}\left(\hat{\Psi}_{1}(0)-\hat{\Psi}_{1}(i \omega)\right)\right| \Sigma\right\rangle \\
& \left.-\left\langle\mathrm{eq}_{\mathrm{M}}\left|\left(\hat{\Psi}_{1}(0)-\hat{\Psi}_{1}(-i \omega)\right) M\left[\mathbf{I}-\hat{\Psi}_{0}(-i \omega) M\right]^{-1}\left(\hat{\Psi}_{1}(0)-\hat{\Psi}_{1}(-i \omega)\right)\right| \Sigma\right\rangle\right) .
\end{aligned}
$$

In the stationary case $\alpha=1$ this gives exactly the result obtained in Eq. (7.32) with help of the WienerKhinchin theorem. 


\subsection{Low-Frequency Behavior: $1 / f$ Noise}

We are interested in the behavior for small $\omega$. Here, we additionally assume that also the first and second moment have an expansion similar to the waiting time

$$
\begin{aligned}
& \hat{\Psi}_{1}(\lambda)=\hat{\Psi}_{1}(0)-\lambda^{\alpha} K_{1}+\mathrm{o}\left(\lambda^{\alpha}\right) \\
& \hat{\Psi}_{2}(\lambda)=\hat{\Psi}_{2}(0)-\lambda^{\alpha} K_{2}+\mathrm{o}\left(\lambda^{\alpha}\right) .
\end{aligned}
$$

Essentially this means, that the first and second moment conditioned on the waiting time have the same order of magnitude for all waiting times. Here, we allow $K_{1}=0$ (e.g., when the ensemble mean of the process is zero), but need $K_{2} \neq 0$. The case that the expansion have different exponents can be treated similarly.

We get again similarly to Eq. (7.11)

$$
\left[\mathbf{I}-\hat{\Psi}_{0}( \pm i \omega) M\right]^{-1}=\frac{|\Sigma\rangle\left\langle\mathrm{eq}_{\mathrm{M}}\right|}{\left\langle\mathrm{eq}_{\mathrm{M}}\left|K_{0}\right| \Sigma\right\rangle( \pm i \omega)^{\alpha}}+\mathrm{o}\left(\frac{1}{|\omega|^{\alpha}}\right) .
$$

With this result, we get in first order (the branch cut is at the negative real axis)

$$
\begin{aligned}
\bar{S}_{\alpha}(\omega) \simeq & \frac{1}{\Gamma(1+\alpha)\left\langle\mathrm{eq}_{\mathrm{M}}\left|K_{0}\right| \Sigma\right\rangle} \frac{1}{\omega^{2}}\left(\left\langle\mathrm{eq}_{\mathrm{M}}\left|(i \omega)^{\alpha} K_{2}+(-i \omega)^{\alpha} K_{2}\right| \Sigma\right\rangle\right. \\
& -\left\langle\mathrm{eq}_{\mathrm{M}}\left|(i \omega)^{\alpha} K_{1} M \frac{|\Sigma\rangle\left\langle\mathrm{eq}_{\mathrm{M}}\right|}{\left\langle\mathrm{eq}_{\mathrm{M}}\left|K_{0}\right| \Sigma\right\rangle(i \omega)^{\alpha}}(i \omega)^{\alpha} K_{1}\right| \Sigma\right\rangle \\
& \left.-\left\langle\mathrm{eq}_{\mathrm{M}}\left|(-i \omega)^{\alpha} K_{1} M \frac{|\Sigma\rangle\left\langle\mathrm{eq}_{\mathrm{M}}\right|}{\left\langle\mathrm{eq}_{\mathrm{M}}\left|K_{0}\right| \Sigma\right\rangle(-i \omega)^{\alpha}}(-i \omega)^{\alpha} K_{1}\right| \Sigma\right\rangle\right) \\
= & \frac{1}{\Gamma(1+\alpha)}\left(\frac{\left\langle\mathrm{eq}_{\mathrm{M}}\left|K_{2}\right| \Sigma\right\rangle}{\left\langle\mathrm{eq}_{\mathrm{M}}\left|K_{0}\right| \Sigma\right\rangle}-\frac{\left\langle\mathrm{eq}_{\mathrm{M}}\left|K_{1}\right| \Sigma\right\rangle^{2}}{\left\langle\mathrm{eq}_{\mathrm{M}}\left|K_{0}\right| \Sigma\right\rangle^{2}}\right) \frac{(i \omega)^{\alpha}+(-i \omega)^{\alpha}}{\omega^{2}}
\end{aligned}
$$

Calculating

$$
\begin{aligned}
(i \omega)^{\alpha}+(-i \omega)^{\alpha} & =(\exp (i \alpha \pi / 2)+\exp (-i \alpha \pi / 2))|\omega|^{\alpha} \\
& =2 \cos (\alpha \pi / 2)|\omega|^{\alpha}
\end{aligned}
$$

gives the expected spectrum for small $\omega$

$$
\bar{S}_{\alpha}(\omega) \simeq \frac{2 \cos (\alpha \pi / 2)}{\Gamma(1+\alpha)}\left(\frac{\left\langle\mathrm{eq}_{\mathrm{M}}\left|K_{2}\right| \Sigma\right\rangle}{\left\langle\mathrm{eq}_{\mathrm{M}}\left|K_{0}\right| \Sigma\right\rangle}-\frac{\left\langle\mathrm{eq}_{\mathrm{M}}\left|K_{1}\right| \Sigma\right\rangle^{2}}{\left\langle\mathrm{eq}_{\mathrm{M}}\left|K_{0}\right| \Sigma\right\rangle^{2}}\right) \frac{1}{|\omega|^{2-\alpha}}
$$

The factor with the $K_{i}$ can be understood by looking at the long-time distribution $\bar{\phi}^{\text {lt }}(x)$ (Eq. (2.15)) Its variance $\sigma_{\mathrm{lt}}^{2}$ is calculated in Appendix $\mathrm{C}$ in terms of the $\left\langle\mathrm{eq}_{\mathrm{M}}\left|K_{n}\right| \Sigma\right\rangle$ (Eq. (C.12)). Using this result allows to rewrite the small frequency behavior of the spectrum as

$$
\bar{S}_{\alpha}(\omega) \simeq \frac{2 \cos (\alpha \pi / 2) \sigma_{\mathrm{lt}}^{2}}{\Gamma(1+\alpha)} \frac{1}{|\omega|^{2-\alpha}} .
$$

Therefore, the expansion Eq. (8.22) is valid as long as $\sigma_{\mathrm{lt}}^{2}>0$. This is the case if for long waiting times, the possible $x$ values do not concentrate on a single value.

In the other cases, one has to go back to Eq. (8.15) and use more terms in the expansion. A typical case for this appears when the signal consists of long waiting times with $x=0$ and burst of finite average length between these waiting times. 


\subsection{Examples}

We illustrate these results with our models. The single state model has

$$
\begin{aligned}
\bar{S}_{\alpha}^{\text {single }}(\omega)= & \frac{1}{\Gamma(1+\alpha) \bar{\tau}_{\text {single }}^{\alpha} \omega^{2}}\left(2 \hat{\psi}_{2}^{\text {single }}(0)-\hat{\psi}_{2}^{\text {single }}(i \omega)-\hat{\psi}_{2}^{\text {single }}(-i \omega)\right. \\
& \left.-\frac{\left(\hat{\psi}_{1}^{\text {single }}(0)-\hat{\psi}_{1}^{\text {single }}(i \omega)\right)^{2}}{1-\hat{\psi}_{0}^{\text {single }}(i \omega)}-\frac{\left(\hat{\psi}_{1}^{\text {single }}(0)-\hat{\psi}_{1}^{\text {single }}(-i \omega)\right)^{2}}{1-\hat{\psi}_{0}^{\text {single }}(-i \omega)}\right) .
\end{aligned}
$$

We want to specialize the distribution $\phi^{\text {single }}(x, t)$ to be equal to the alternating process, but not with alternating between the \pm states but by randomly selecting them. This is done by choosing

$$
\phi^{\text {single }}(x, t)=\frac{1}{2}\left(\delta\left(x-I_{+}\right)+\delta\left(x-I_{-}\right)\right) \psi^{\text {alt }}(t) .
$$

Especially, the state averaged distribution $\bar{\phi}(x, t)$ and the long time distribution $\bar{\phi}^{\text {lt }}(x)$ are the same. With

$$
\hat{\psi}_{n}^{\text {single }}(\lambda)=\frac{I_{+}^{n}+I_{-}^{n}}{2} \hat{\psi}^{\text {alt }}(\lambda)
$$

we get in this case

$$
\bar{S}_{\alpha}^{\text {single }}(\omega)=\frac{\left(I_{+}-I_{-}\right)^{2}}{4 \Gamma(1+\alpha) \bar{\tau}^{\alpha} \omega^{2}}\left(2-\hat{\psi}^{\text {alt }}(i \omega)-\hat{\psi}^{\text {alt }}(-i \omega)\right)
$$

The low frequency behavior comes from expansion $\hat{\psi}^{\text {alt }}(\lambda) \simeq 1-\bar{\tau}^{\alpha} \lambda^{\alpha}$ and reads

$$
\bar{S}_{\alpha}^{\text {single }}(\omega) \simeq \frac{\left(I_{+}-I_{-}\right)^{2} \cos (\alpha \pi / 2)}{2 \Gamma(1+\alpha)} \frac{1}{\omega^{2-\alpha}} .
$$

This result could also have been obtain by the long time distribution in this case which is

$$
\bar{\phi}^{\mathrm{lt}}(x)=\frac{1}{2}\left(\delta\left(x-I_{+}\right)+\delta\left(x-I_{-}\right)\right)
$$

with variance

$$
\sigma_{\mathrm{lt}}^{2}=\left(\frac{I_{+}-I_{-}}{2}\right)^{2}
$$

For the alternating model, we get

$$
\begin{aligned}
\bar{S}_{\alpha}^{\mathrm{alt}}(\omega)= & \frac{1}{\Gamma(1+\alpha) \bar{\tau}^{\alpha}} \frac{1}{\omega^{2}}\left(\left(2-\hat{\psi}^{\mathrm{alt}}(i \omega)-\hat{\psi}^{\mathrm{alt}}(-i \omega)\right)\left\langle\mathrm{eq} \mathrm{M}\left|\left(\begin{array}{cc}
I_{+}^{2} & 0 \\
0 & I_{-}^{2}
\end{array}\right)\right| \Sigma\right\rangle\right. \\
& -\frac{\left(1-\hat{\psi}^{\mathrm{alt}}(i \omega)\right)^{2}}{1-\hat{\psi}^{\mathrm{alt}}(i \omega)^{2}}\left\langle\mathrm{eq}_{\mathrm{M}}\left|\left(\begin{array}{cc}
0 & I_{+} \\
I_{-} & 0
\end{array}\right)\left(\begin{array}{cc}
1 & \hat{\psi}^{\mathrm{alt}}(i \omega) \\
\hat{\psi}^{\mathrm{alt}}(i \omega) & 1
\end{array}\right)\left(\begin{array}{cc}
I_{+} & 0 \\
0 & I_{-}
\end{array}\right)\right| \Sigma\right\rangle \\
& \left.-\frac{\left(1-\hat{\psi}^{\mathrm{alt}}(-i \omega)\right)^{2}}{1-\hat{\psi}^{\mathrm{alt}}(-i \omega)^{2}}\left\langle\mathrm{eq}_{\mathrm{M}}\left|\left(\begin{array}{cc}
0 & I_{+} \\
I_{-} & 0
\end{array}\right)\left(\begin{array}{cc}
1 & \hat{\psi}^{\mathrm{alt}}(-i \omega) \\
\hat{\psi}^{\mathrm{alt}}(-i \omega) & 1
\end{array}\right)\left(\begin{array}{cc}
I_{+} & 0 \\
0 & I_{-}
\end{array}\right)\right| \Sigma\right\rangle\right) \\
= & \frac{\left(I_{+}-I_{-}\right)^{2}}{2 \Gamma(1+\alpha) \bar{\tau}^{\alpha} \omega^{2}}\left(\frac{1-\hat{\psi}^{\mathrm{alt}}(i \omega)}{1+\hat{\psi}^{\mathrm{alt}}(i \omega)}+\frac{1-\hat{\psi}^{\mathrm{alt}}(-i \omega)}{1+\hat{\psi}^{\mathrm{alt}}(-i \omega)}\right) .
\end{aligned}
$$

This is clearly distinct from the spectrum obtained for random switching. But the low frequency behavior is again

$$
\bar{S}_{\alpha}^{\text {alt }}(\omega) \simeq \frac{\left(I_{+}-I_{-}\right)^{2} \cos (\alpha \pi / 2)}{2 \Gamma(1+\alpha)} \frac{1}{\omega^{2-\alpha}} .
$$


which is clear as the alternating models also has the same long time distribution

$$
\bar{\phi}^{\mathrm{lt}}(x)=\frac{1}{2}\left(\delta\left(x-I_{+}\right)+\delta\left(x-I_{-}\right)\right) .
$$

We also want to discuss here the burst model, as its long time distribution is $\bar{\phi}^{\text {tt }}(x)=\delta\left(x-I_{-}\right)$with variance $\sigma_{\mathrm{lt}}^{2}=0$ which generates a different low frequency behavior. The expected value of the spectrum is

$$
\begin{aligned}
\bar{S}_{\alpha}^{\text {burst }}(\omega)= & \frac{\left(I_{+}-I_{-}\right)^{2}}{2} \frac{1}{\Gamma(1+\alpha)\left\langle\mathrm{eq}_{\mathrm{M}}\left|K_{0}\right| \Sigma\right\rangle} \frac{1}{\omega^{2}} \\
& \times\left(\frac{\left(1-\hat{\psi}_{+}^{\text {burst }}(i \omega)\right)\left(1-\hat{\psi}_{-}^{\text {burst }}(i \omega)\right)}{1-\hat{\psi}_{+}^{\text {burst }}(i \omega) \hat{\psi}_{-}^{\text {burst }}(i \omega)}+\frac{\left(1-\hat{\psi}_{+}^{\text {burst }}(-i \omega)\right)\left(1-\hat{\psi}_{-}^{\text {burst }}(-i \omega)\right)}{1-\hat{\psi}_{+}^{\text {burst }}(-i \omega) \hat{\psi}_{-}^{\text {burst }}(-i \omega)}\right)
\end{aligned}
$$

which still is an exact result without any reference to the exact forms of $\hat{\psi}_{+}^{\text {burst }}(\lambda)$ and $\hat{\psi}_{-}^{\text {burst }}(\lambda)$ (i.e., setting $\hat{\psi}_{+}^{\text {burst }}(\lambda)=\hat{\psi}_{-}^{\text {burst }}(\lambda)=\psi^{\text {alt }}(\lambda)$ gives the result $\left.\bar{S}_{\alpha}^{\text {alt }}(\omega)\right)$. Now, we remember our assumptions Eq. (2.30)

$$
\begin{aligned}
& \hat{\psi}_{+}^{\text {burst }}(\lambda)=1-c_{+} \lambda^{\tilde{\alpha}}+o\left(\lambda^{\tilde{\alpha}}\right) \\
& \hat{\psi}_{-}^{\text {burst }}(\lambda)=1-c_{-} \lambda^{\alpha}+o\left(\lambda^{\alpha}\right) .
\end{aligned}
$$

with $0<\alpha<\tilde{\alpha}<1$. And Eq. (2.31) for $\tilde{\alpha}=1$ and $0<\tilde{\beta} \leq 1$

$$
\begin{aligned}
& \hat{\psi}_{+}^{\text {burst }}(\lambda)=1-c_{+} \lambda+c_{+}^{(2)} \lambda^{1+\tilde{\beta}}+o\left(\lambda^{1+\tilde{\beta}}\right) \\
& \hat{\psi}_{-}^{\text {burst }}(\lambda)=1-c_{-} \lambda^{\alpha}+o\left(\lambda^{\alpha}\right) .
\end{aligned}
$$

The low frequency behavior of the spectrum is then for $\tilde{\alpha}<1$ (note: $\bar{\tau}^{\alpha}=\left\langle\mathrm{eq}_{\mathrm{M}}\left|K_{0}\right| \Sigma\right\rangle=c_{-} / 2$ )

$$
\begin{aligned}
\bar{S}_{\alpha}^{\text {burst }}(\omega) & =\frac{\left(I_{+}-I_{-}\right)^{2}}{\Gamma(1+\alpha) c_{-}} \frac{1}{\omega^{2}}\left(\frac{c_{-} c_{+}(i \omega)^{\alpha+\tilde{\alpha}}+\mathrm{o}\left(\omega^{\alpha+\tilde{\alpha}}\right)}{c_{-}(i \omega)^{\alpha}+\mathrm{o}\left(\omega^{\alpha}\right)}+\frac{c_{-} c_{+}(-i \omega)^{\alpha+\tilde{\alpha}}+\mathrm{o}\left(\omega^{\alpha+\tilde{\alpha}}\right)}{c_{-}(-i \omega)^{\alpha}+\mathrm{o}\left(\omega^{\alpha}\right)}\right) \\
& =\left(I_{+}-I_{-}\right)^{2} \frac{2 \cos (\tilde{\alpha} \pi / 2)}{\Gamma(1+\alpha)} \frac{c_{+}}{c_{-}} \frac{1}{\omega^{2-\tilde{\alpha}}}+\mathrm{o}\left(\omega^{\tilde{\alpha}-2}\right) .
\end{aligned}
$$

The $1 / f$ noise is determined by the larger exponent $\tilde{\alpha}$. However, the decay of the spectrum is still given by $\alpha:\left\langle S_{T}(\omega)\right\rangle \simeq T^{\alpha-1} \bar{S}_{\alpha}^{\text {burst }}(\omega)$.

For $\tilde{\alpha}=1$ we get

$$
\begin{aligned}
\bar{S}_{\alpha}^{\text {burst }}(\omega) & =\frac{\left(I_{+}-I_{-}\right)^{2}}{\Gamma(1+\alpha) c_{-}} \frac{1}{\omega^{2}}\left(\frac{1-\hat{\psi}_{+}^{\text {burst }}(i \omega)}{1+\hat{\psi}_{-}^{\text {burst }}(i \omega) \frac{1-\hat{\psi}_{+}^{\text {burst }}(i \omega)}{1-\hat{\psi}_{-}^{\text {burst }}(i \omega)}}+\frac{1-\hat{\psi}_{+}^{\text {burst }}(-i \omega)}{1+\hat{\psi}_{-}^{\text {burst }}(-i \omega) \frac{1-\hat{\psi}_{+}^{\text {burst }}(-i \omega)}{1-\hat{\psi}_{-}^{\text {burst }}(-i \omega)}}\right) \\
& =\frac{\left(I_{+}-I_{-}\right)^{2}}{\Gamma(1+\alpha) c_{-}} \frac{1}{\omega^{2}}\left(\frac{c_{+} i \omega-c_{+}^{(2)}(i \omega)^{1+\tilde{\beta}}+\mathrm{o}\left(\omega^{1+\tilde{\beta}}\right)}{1+\frac{c_{+}}{c_{-}}(i \omega)^{1-\alpha}+\mathrm{o}\left(\omega^{1-\alpha}\right)}+\frac{-c_{+} i \omega-c_{+}^{(2)}(-i \omega)^{1+\tilde{\beta}}+\mathrm{o}\left(\omega^{1+\tilde{\beta}}\right)}{1+\frac{c_{+}}{c_{-}}(-i \omega)^{1-\alpha}+\mathrm{o}\left(\omega^{1-\alpha}\right)}\right) \\
= & \frac{\left(I_{+}-I_{-}\right)^{2}}{\Gamma(1+\alpha) c_{-}} \frac{1}{\omega^{2}}\left(2 \frac{c_{+}^{2}}{c_{-}} \cos (\alpha \pi / 2) \omega^{2-\alpha}+2 c_{+}^{(2)} \sin (\tilde{\beta} \pi / 2) \omega^{1+\tilde{\beta}}+\mathrm{o}\left(\omega^{2-\alpha}\right)+\mathrm{o}\left(\omega^{1+\tilde{\beta}}\right)\right) \\
= & \begin{cases}\left(I_{+}-I_{-}\right)^{2} \frac{2 \cos (\alpha \pi / 2)}{\Gamma(1+\alpha)} \frac{c_{+}^{2}}{c_{-}^{2}} \frac{1}{\omega^{\alpha}}+\mathrm{o}\left(\omega^{-\alpha}\right) & \text { for } \alpha>1-\tilde{\beta} \\
\left(I_{+}-I_{-}\right)^{2} \frac{2 \sin (\tilde{\beta} \pi / 2)}{\Gamma(1+\alpha)} \frac{c_{+}^{(2)}}{c_{-}} \frac{1}{\omega^{1-\tilde{\beta}}}+\mathrm{o}\left(\omega^{\tilde{\beta}-1}\right) & \text { for } \alpha<1-\tilde{\beta} .\end{cases}
\end{aligned}
$$

We have two contributions for the $1 / f$ noise and we see the dominant one, depending on the values of the parameters $\alpha$ and $\tilde{\beta}$. 


\section{Correlations of the Spectrum}

It has been established that for $\alpha<1$ the spectrum is not reproducible even after smoothing [40]. The correlations of the spectrum help to understand the randomness in its determination.

In this section we want to determine the stochastic long time behavior of the vector of observed spectra

$$
\left(S_{T}\left(\omega_{1}\right), \ldots, S_{T}\left(\omega_{r}\right)\right)
$$

where the $\omega_{i}>0$ are pairwise disjoint frequencies. This will be done by the method of moments [11], i.e., we are calculating the moments $\left(q_{i}=0,1,2, \ldots\right)$

$$
\left\langle S_{T}\left(\omega_{1}\right)^{q_{1}} \cdots S_{T}\left(\omega_{r}\right)^{q_{r}}\right\rangle\langle\text { init }|
$$

and reconstruct the probability distribution from these moments.

In a first step, it is easier to allow same frequencies

$$
\left\langle S_{T}\left(\bar{\omega}_{1}\right) \cdots S_{T}\left(\bar{\omega}_{p}\right)\right\rangle_{\langle\text {init }|}=\left\langle S_{T}\left(\omega_{1}\right)^{q_{1}} \cdots S_{T}\left(\omega_{r}\right)^{q_{r}}\right\rangle_{\langle\text {init }|}
$$

such that $\omega_{j}$ appears $q_{j}$ times in the $\bar{\omega}_{1}, \ldots, \bar{\omega}_{p}$. We have then $p=q_{1}+\cdots+q_{r}$.

Similar to the last Section 8 we use the trick of introducing different times

$$
T^{p}\left\langle S_{T}\left(\bar{\omega}_{1}\right) \cdots S_{T}\left(\bar{\omega}_{p}\right)\right\rangle_{\langle\text {init }|}=\left.\left\langle F_{T_{1}}\left(-\bar{\omega}_{1}\right) F_{T_{2}}\left(\bar{\omega}_{1}\right) \cdots F_{T_{2 p-1}}\left(-\bar{\omega}_{p}\right) F_{T_{2 p}}\left(\bar{\omega}_{p}\right)\right\rangle_{\langle\text {init }|}\right|_{T_{1}=\cdots=T_{2 p}=T} .
$$

We introduce further the notation

$$
\tilde{\omega}_{1}=\bar{\omega}_{1}, \tilde{\omega}_{2}=-\bar{\omega}_{1}, \ldots, \tilde{\omega}_{2 p-1}=\bar{\omega}_{p}, \tilde{\omega}_{2 p}=-\bar{\omega}_{p}
$$

and similar to the definition of $\Lambda_{\mathcal{V}}$

$$
\tilde{\Omega}_{\mathcal{J}}=\sum_{j \in \mathcal{J}} \tilde{\omega}_{j}
$$

We have then

$$
T^{p}\left\langle S_{T}\left(\bar{\omega}_{1}\right) \cdots S_{T}\left(\bar{\omega}_{p}\right)\right\rangle_{\langle\text {init }|}=\left.\left\langle F_{T_{1}}\left(-\tilde{\omega}_{1}\right) F_{T_{2}}\left(-\tilde{\omega}_{2}\right) \cdots F_{T_{2 p-1}}\left(-\tilde{\omega}_{2 p-1}\right) F_{T_{2 p}}\left(-\tilde{\omega}_{2 p}\right)\right\rangle_{\langle\text {init }|}\right|_{T_{1}=\cdots=T_{2 p}=T} .
$$

The long time behavior is captured by the limit

$$
\left.\lim _{\zeta \rightarrow 0} \zeta^{p \alpha}\left\langle F_{T_{1} / \zeta}\left(-\tilde{\omega}_{1}\right) \cdots F_{T_{2 p} / \zeta}\left(-\tilde{\omega}_{2 p}\right)\right\rangle_{\langle\text {init }|}\right|_{T_{1}=\cdots=T_{2 p}=T} .
$$

The scaling $\zeta^{p \alpha}$ stems from the scaling $S_{T}\left(\bar{\omega}_{i}\right) \sim T^{\alpha-1}$ for large $T$. The Laplace transform of this expression (with the Laplace pairs $\lambda_{i} \leftrightarrow T_{i}$ ) is

$$
\begin{aligned}
& \mathrm{L}\left[\zeta^{p \alpha}\left\langle F_{T_{1} / \zeta}\left(-\tilde{\omega}_{1}\right) \cdots F_{T_{2 p} / \zeta}\left(-\tilde{\omega}_{2 p}\right)\right\rangle_{\langle\text {init }|}\right] \\
& =\zeta^{p \alpha} \int_{0}^{\infty} \mathrm{d} T_{1} \cdots \int_{0}^{\infty} \mathrm{d} T_{2 p} e^{-\lambda_{1} T_{1}-\cdots-\lambda_{2 p} T_{2 p}} \\
& \times \int_{0}^{T_{1} / \zeta} \mathrm{d} t_{1} \cdots \int_{0}^{T_{2 p} / \zeta} \mathrm{d} t_{2 p} e^{-i\left(\tilde{\omega}_{1} t_{1}+\cdots+\tilde{\omega}_{2 p} t_{2 p}\right)}\left\langle X\left(t_{1}\right) \cdots X\left(t_{2 p}\right)\right\rangle_{\langle\text {init }|} \\
& =\frac{\zeta^{p \alpha}}{\lambda_{1} \cdots \lambda_{2 p}}\left\langle\operatorname{init} \mid \hat{C}_{\{1, \ldots, 2 p\}}\left(\left\{\zeta \lambda_{k}+i \tilde{\omega}_{k}\right\}\right)\right\rangle \text {. }
\end{aligned}
$$

Before we proceed to evaluate the last line of Eq. (9.9), we will have a more detailed look at the term $\left|\hat{C}_{\{1, \ldots, 2 p\}}\left(\left\{\zeta \lambda_{k}+i \tilde{\omega}_{k}\right\}\right)\right\rangle$. 


\subsection{Direct Calculation of the Correlation}

The correlations can be calculated by the recursion Eq. (5.9)

$$
\left|\hat{C}_{\mathcal{L}_{\mathrm{i}}}\left(\left\{\lambda_{k}\right\}\right)\right\rangle=\left[\mathbf{I}-\hat{\Psi}_{0}\left(\Lambda_{\mathcal{L}_{\mathrm{i}}}\right) M\right]^{-1} \sum_{\mathcal{L}_{\mathrm{f}}: \mathcal{L}_{\mathrm{i}} \supsetneq \mathcal{L}_{\mathrm{f}}} \hat{R}_{\mathcal{L}_{\mathrm{i}} \mathcal{L}_{\mathrm{f}}}\left(\left\{\lambda_{k}\right\}\right)\left|\hat{C}_{\mathcal{L}_{\mathrm{f}}}\left(\left\{\lambda_{k}\right\}\right)\right\rangle
$$

where the operators $\hat{R}_{\mathcal{L}_{\mathrm{i}} \mathcal{L}_{\mathrm{f}}}\left(\left\{\lambda_{k}\right\}\right)$ are defined in Eq. (5.8).

For $\mathcal{L}_{\mathrm{f}}=\emptyset$ the recursion ends $\left|\hat{C}_{0}(\{\})\right\rangle=|\Sigma\rangle$. All other terms can be iterated again, i.e., we get for the $n$-point correlation $\left(\mathcal{L}_{1}=\mathcal{L}_{\mathrm{i}}\right)$

$$
\begin{aligned}
& \left|\hat{C}_{\mathcal{L}_{1}}\left(\left\{\lambda_{k}\right\}\right)\right\rangle=\sum_{\mathcal{L}_{2}: \mathcal{L}_{1} \supsetneq \mathcal{L}_{2}}\left[\mathbf{I}-\hat{\Psi}_{0}\left(\Lambda_{\mathcal{L}_{1}}\right) M\right]^{-1} \hat{R}_{\mathcal{L}_{1} \mathcal{L}_{2}}\left(\left\{\lambda_{k}\right\}\right)\left|\hat{C}_{\mathcal{L}_{2}}\left(\left\{\lambda_{k}\right\}_{k \in \mathcal{L}_{2}}\right)\right\rangle \\
& =\left[\mathbf{I}-\hat{\Psi}_{0}\left(\Lambda_{\mathcal{L}_{1}}\right) M\right]^{-1} \hat{R}_{\mathcal{L}_{1} \emptyset}\left(\left\{\lambda_{k}\right\}\right)|\Sigma\rangle \\
& +\sum_{\substack{\mathcal{L}_{2}, \mathcal{L}_{3} \\
\mathcal{L}_{1} \supsetneq \mathcal{L}_{2} \supsetneq \mathcal{L}_{3}}}\left[\mathbf{I}-\hat{\Psi}_{0}\left(\Lambda_{\mathcal{L}_{1}}\right) M\right]^{-1} \hat{R}_{\mathcal{L}_{1} \mathcal{L}_{2}}\left(\left\{\lambda_{k}\right\}\right)\left[\mathbf{I}-\hat{\Psi}_{0}\left(\Lambda_{\mathcal{L}_{2}}\right) M\right]^{-1} \hat{R}_{\mathcal{L}_{2} \mathcal{L}_{3}}\left(\left\{\lambda_{k}\right\}\right)\left|\hat{C}_{\mathcal{L}_{3}}\left(\left\{\lambda_{k}\right\}\right)\right\rangle \\
& =\left[\mathbf{I}-\hat{\Psi}_{0}\left(\Lambda_{\mathcal{L}_{1}}\right) M\right]^{-1} \hat{R}_{\mathcal{L}_{1} \emptyset}\left(\left\{\lambda_{k}\right\}\right)|\Sigma\rangle \\
& +\sum_{\substack{\mathcal{L}_{2} \\
\mathcal{L}_{1} \supsetneq \mathcal{L}_{2} \supsetneq \emptyset}}\left[\mathbf{I}-\hat{\Psi}_{0}\left(\Lambda_{\mathcal{L}_{1}}\right) M\right]^{-1} \hat{R}_{\mathcal{L}_{1} \mathcal{L}_{2}}\left(\left\{\lambda_{k}\right\}\right)\left[\mathbf{I}-\hat{\Psi}_{0}\left(\Lambda_{\mathcal{L}_{2}}\right) M\right]^{-1} \hat{R}_{\mathcal{L}_{2} \emptyset}\left(\left\{\lambda_{k}\right\}\right)|\Sigma\rangle \\
& +\sum_{\substack{\mathcal{L}_{2}, \mathcal{L}_{3}, \mathcal{L}_{4} \\
\mathcal{L}_{1} \supsetneq \mathcal{L}_{2} \supsetneq \mathcal{L}_{3} \supsetneq \mathcal{L}_{4}}}\left[\mathbf{I}-\hat{\Psi}_{0}\left(\Lambda_{\mathcal{L}_{1}}\right) M\right]^{-1} R_{\mathcal{L}_{1} \mathcal{L}_{2}}\left(\left\{\lambda_{k}\right\}\right) \cdots R_{\mathcal{L}_{3} \mathcal{L}_{4}}\left(\left\{\lambda_{k}\right\}\right)\left|\hat{C}_{\mathcal{L}_{4}}\left(\left\{\lambda_{k}\right\}\right)\right\rangle .
\end{aligned}
$$

The last sum $\sum_{\mathcal{L}_{1} \mathcal{L}_{2}, \mathcal{L}_{3}, \mathcal{L}_{4}}$ is taken over all sets $\mathcal{L}_{2}, \mathcal{L}_{3}$ and $\mathcal{L}_{4}$ which form a nested sequence of true subsets: $\mathcal{L}_{\mathrm{i}}=\mathcal{L}_{1} \supsetneq \mathcal{L}_{1} \supsetneq \mathcal{L}_{2} \supsetneq \mathcal{L}_{2} \supsetneq \mathcal{L}_{3} \supsetneq \mathcal{L}_{4}$. As long as the last set is not empty, one can continue to apply the recursion relation Eq. (5.9). Iterating this until it is no further possible to apply Eq. (5.9) leaves us with a sum over all possible nested sequences of true subsets of $\mathcal{L}_{\mathrm{i}} \mathcal{L}_{\mathrm{i}}=\mathcal{L}_{1} \supsetneq \mathcal{L}_{2} \supsetneq \cdots \supsetneq \mathcal{L}_{s} \supsetneq \emptyset$ of length $s$

$$
\left|\hat{C}_{\mathcal{L}_{\mathrm{i}}}\left(\left\{\lambda_{k}\right\}\right)\right\rangle=\sum_{s} \sum_{\substack{\mathcal{L}_{2}, \ldots, \mathcal{L}_{s} \\ \mathcal{L}_{1}=\mathcal{L}_{\mathrm{i}} \\ \mathcal{L}_{1} \supsetneq \mathcal{L}_{2} \supsetneq \cdots \supsetneq \mathcal{L}_{s} \supsetneq \emptyset}}\left[\mathbf{I}-\hat{\Psi}_{0}\left(\Lambda_{\mathcal{L}_{1}}\right) M\right]^{-1} \hat{R}_{\mathcal{L}_{1} \mathcal{L}_{2}}\left(\left\{\lambda_{k}\right\}\right)\left[\mathbf{I}-\hat{\Psi}_{0}\left(\Lambda_{\mathcal{L}_{2}}\right) M\right]^{-1} \cdots \hat{R}_{\mathcal{L}_{s} \emptyset}\left(\left\{\lambda_{k}\right\}\right)|\Sigma\rangle .
$$

The first sum, is the sum over the different length of the nested sequences of subsets.

Let us construct the two-time correlation $\left|\hat{C}_{\{1,2\}}\left(\left\{\lambda_{k}\right\}\right)\right\rangle$ to illustrate this construction. We are looking for nested sequences of subsets of $\{1,2\}$. We have one sequences of length $s=1$

$$
\{1,2\} \supsetneq \emptyset \quad \Rightarrow \quad \mathcal{L}_{1}=\{1,2\}
$$

and two of length $s=2$

$$
\begin{array}{lll}
\{1,2\} \supsetneq\{1\} \supsetneq \emptyset \quad & \Rightarrow \quad \mathcal{L}_{1}=\{1,2\}, \mathcal{L}_{2}=\{1\} \\
\{1,2\} \supsetneq\{2\} \supsetneq \emptyset \quad & \Rightarrow \quad \mathcal{L}_{1}=\{1,2\}, \mathcal{L}_{2}=\{2\} .
\end{array}
$$




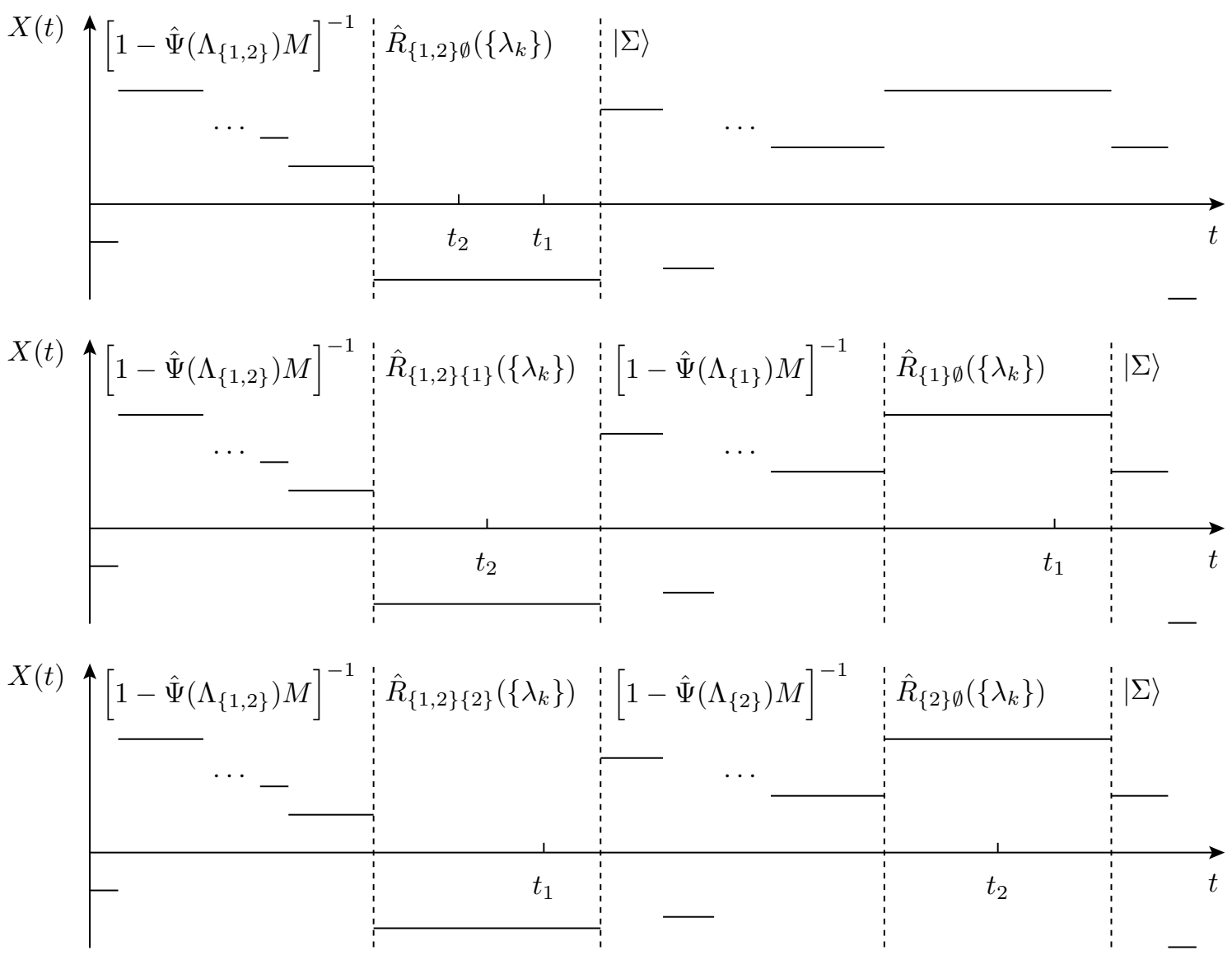

Figure 4. Explanation of the terms in Eq. (9.15), based on Figure 3: The first term describes the situation that $t_{1}$ and $t_{2}$ are in the same epoch (which can be any epoch after the start of the process). The contribution of any number of epochs before $t_{1}$ or $t_{2}$ is described by $\left[\mathbf{I}-\hat{\Psi}_{0}\left(\Lambda_{\{1,2\}}\right) M\right]^{-1}$. The contribution of the epoch containing $t_{1}$ and $t_{2}$ is given by $\hat{R}_{\{1,2\} \emptyset}\left(\left\{\lambda_{k}\right\}\right)$. After this epoch, no epoch contributes any more which is given by $|\Sigma\rangle$ - a summation over all possible internal states. The second term corresponds to the situation that $t_{1}$ is in a later epoch than $t_{2}$. The contribution of any number of epochs before $t_{1}$ or $t_{2}$ is described again by $\left[\mathbf{I}-\hat{\Psi}_{0}\left(\Lambda_{\{1,2\}}\right) M\right]^{-1}$. The contribution of the epoch containing only $t_{2}$ by $\hat{R}_{\{1,2\}\{1\}}\left(\left\{\lambda_{k}\right\}\right)$. After this the same terms reappear only for the time $t_{1}$. The third term is just the second term with the indices 1 and 2 exchanged.

These pictures can be generalized to an $n$-point correlation described by Eq. (9.12).

Therefore, we have three contributions to $\left|\hat{C}_{\{1,2\}}\left(\left\{\lambda_{k}\right\}\right)\right\rangle$ corresponding exactly to these sequences

$$
\begin{aligned}
\left|\hat{C}_{\{1,2\}}\left(\left\{\lambda_{k}\right\}\right)\right\rangle= & {\left[\mathbf{I}-\hat{\Psi}_{0}\left(\Lambda_{\{1,2\}}\right) M\right]^{-1} \hat{R}_{\{1,2\} \emptyset}\left(\left\{\lambda_{k}\right\}\right)|\Sigma\rangle } \\
& +\left[\mathbf{I}-\hat{\Psi}_{0}\left(\Lambda_{\{1,2\}}\right) M\right]^{-1} \hat{R}_{\{1,2\}\{1\}}\left(\left\{\lambda_{k}\right\}\right)\left[\mathbf{I}-\hat{\Psi}_{0}\left(\Lambda_{\{1\}}\right) M\right]^{-1} \hat{R}_{\{1\} \emptyset}\left(\left\{\lambda_{k}\right\}\right)|\Sigma\rangle \\
& +\left[\mathbf{I}-\hat{\Psi}_{0}\left(\Lambda_{\{1,2\}}\right) M\right]^{-1} \hat{R}_{\{1,2\}\{2\}}\left(\left\{\lambda_{k}\right\}\right)\left[\mathbf{I}-\hat{\Psi}_{0}\left(\Lambda_{\{2\}}\right) M\right]^{-1} \hat{R}_{\{2\} \emptyset}\left(\left\{\lambda_{k}\right\}\right)|\Sigma\rangle
\end{aligned}
$$


which gives again Eq. (5.11) when plugging in the expressions for the $\hat{R}_{\mathcal{L}_{\mathrm{i}} \mathcal{L}_{\mathrm{f}}}\left(\left\{\lambda_{k}\right\}\right)$. The different terms are explained graphically in Figure 4.

\subsection{Correlation Functions for the Spectrum}

Using this result with the correlation function needed for the spectrum Eq. (9.8) and (9.9)

$$
\begin{aligned}
\lim _{\zeta \rightarrow 0} \zeta^{p \alpha}\left\langle\operatorname{init} \mid \hat{C}_{\{1, \ldots, 2 p\}}\left(\left\{\zeta \lambda_{k}+i \tilde{\omega}_{k}\right\}\right)\right\rangle & =\lim _{\zeta \rightarrow 0} \zeta^{p \alpha} \sum_{s} \sum_{\begin{array}{c}
\mathcal{L}_{2}, \ldots, \mathcal{L}_{s} \\
\mathcal{L}_{1}=\{1, \ldots, 2 p\} \\
\mathcal{L}_{1} \supsetneq \mathcal{L}_{2} \supsetneq \cdots \supsetneq \mathcal{L}_{s} \supsetneq \emptyset
\end{array}}\langle\operatorname{init}|\left[\mathbf{I}-\hat{\Psi}_{0}\left(\zeta \Lambda_{\mathcal{L}_{1}}+i \tilde{\Omega}_{\mathcal{L}_{1}}\right) M\right]^{-1} \hat{R}_{\mathcal{L}_{1} \mathcal{L}_{2}}\left(\left\{\zeta \lambda_{k}+i \tilde{\omega}_{k}\right\}\right) \\
& \quad\left[\mathbf{I}-\hat{\Psi}_{0}\left(\zeta \Lambda_{\mathcal{L}_{2}}+i \tilde{\Omega}_{\mathcal{L}_{2}}\right) M\right]^{-1} \cdots\left[\mathbf{I}-\hat{\Psi}_{0}\left(\zeta \Lambda_{\mathcal{L}_{s}}+i \tilde{\Omega}_{\mathcal{L}_{s}}\right) M\right]^{-1} \hat{R}_{\mathcal{L}_{s} \emptyset}\left(\left\{\zeta \lambda_{k}+i \tilde{\omega}_{k}\right\}\right)|\Sigma\rangle .
\end{aligned}
$$

The only terms that can become singular as $\zeta \rightarrow 0$ are the terms

$$
\left[\mathbf{I}-\hat{\Psi}_{0}\left(\zeta \Lambda_{\mathcal{L}_{j}}+i \tilde{\Omega}_{\mathcal{L}_{j}}\right) M\right]^{-1}
$$

Using Eq. (8.10) and the fact that that we do not have a singularity for $\tilde{\Omega}_{\mathcal{L}_{j}} \neq 0$

$$
\left[\mathbf{I}-\hat{\Psi}_{0}\left(\zeta \Lambda_{\mathcal{L}_{j}}+i \tilde{\Omega}_{\mathcal{L}_{j}}\right) M\right]^{-1}=\left\{\begin{array}{ll}
{\left[\mathbf{I}-\hat{\Psi}_{0}\left(i \tilde{\Omega}_{\mathcal{L}_{j}}\right) M\right]^{-1}+\mathrm{o}(1)} & \text { for } \tilde{\Omega}_{\mathcal{L}_{j}} \neq 0 \\
\frac{|\Sigma\rangle\langle\mathrm{eq}}{\bar{\tau}^{\alpha} \Lambda_{\mathcal{L}_{j}}^{\alpha}} \zeta^{-\alpha}+\mathrm{o}\left(\zeta^{-\alpha}\right) & \text { for } \tilde{\Omega}_{\mathcal{L}_{j}}=0
\end{array} \quad \text { as } \zeta \rightarrow 0 .\right.
$$

This motivates a regrouping of Eq. (9.16) in singular terms and non-singular terms for $\zeta \rightarrow 0$. We introduce a new operator $\tilde{R}_{\mathcal{L}_{\mathrm{i}} \mathcal{L}_{\mathrm{f}}}\left(\left\{\lambda_{k}\right\} ;\left\{\tilde{\omega}_{k}\right\}\right)$ which is

$$
\begin{aligned}
& \tilde{R}_{\mathcal{L}_{\mathrm{i}} \mathcal{L}_{\mathrm{f}}}\left(\left\{\lambda_{k}\right\} ;\left\{\tilde{\omega}_{k}\right\}\right) \\
& =\sum_{s \geq 2} \sum_{\substack{\mathcal{L}_{2}, \ldots, \mathcal{L}_{s} \\
\mathcal{L}_{\mathfrak{i}}=\mathcal{L}_{1} \supsetneq \mathcal{L}_{2} \supsetneq \cdots, \mathcal{L}_{s}=\mathcal{L}_{\mathrm{f}} \\
\tilde{\Omega}_{\mathcal{L}_{j}} \neq 0 \text { for } j=2, \ldots, s-1}} \hat{R}_{\mathcal{L}_{1} \mathcal{L}_{2}}\left(\left\{\lambda_{k}\right\}\right)\left[\mathbf{I}-\hat{\Psi}_{0}\left(\Lambda_{\mathcal{L}_{2}}\right) M\right]^{-1} \hat{R}_{\mathcal{L}_{2} \mathcal{L}_{3}}\left(\left\{\lambda_{k}\right\}\right) \cdots \\
& \times\left[\mathbf{I}-\hat{\Psi}_{0}\left(\Lambda_{\mathcal{L}_{s-1}}\right) M\right]^{-1} \hat{R}_{\mathcal{L}_{s-1} \mathcal{L}_{s}}\left(\left\{\lambda_{k}\right\}\right)+\hat{R}_{\mathcal{L}_{\mathrm{i}} \mathcal{L}_{\mathrm{f}}}\left(\left\{\lambda_{k}\right\}\right) \quad \text { for } \tilde{\Omega}_{\mathcal{L}_{\mathrm{f}}}=0
\end{aligned}
$$

and

$$
=0 \quad \text { for } \tilde{\Omega}_{\mathcal{L}_{\mathrm{f}}} \neq 0
$$

With this new definition, we can rewrite Eq. (9.12) (with the arguments from (9.16))

$$
\begin{aligned}
& \left|\hat{C}_{\mathcal{L}_{\mathrm{i}}}\left(\left\{\zeta \lambda_{k}+i \tilde{\omega}_{k}\right\}\right)\right\rangle \\
& =\sum_{s} \sum_{\substack{\mathcal{L}_{2}, \ldots, \mathcal{L}_{s} \\
\mathcal{L}_{\mathrm{i}}=\mathcal{L}_{1} \supsetneq \mathcal{L}_{2} \supsetneq \cdots \supsetneq \mathcal{L}_{s} \supsetneq \emptyset}}\left[\mathbf{I}-\hat{\Psi}_{0}\left(\zeta \Lambda_{\mathcal{L}_{1}}+i \tilde{\Omega}_{\mathcal{L}_{1}}\right) M\right]^{-1} \tilde{R}_{\mathcal{L}_{1} \mathcal{L}_{2}}\left(\left\{\zeta \lambda_{k}+i \tilde{\omega}_{k}\right\} ;\left\{\tilde{\omega}_{k}\right\}\right) \\
& {\left[\mathbf{I}-\hat{\Psi}_{0}\left(\zeta \Lambda_{\mathcal{L}_{2}}+i \tilde{\Omega}_{\mathcal{L}_{2}}\right) M\right]^{-1} \cdots\left[\mathbf{I}-\hat{\Psi}_{0}\left(\zeta \Lambda_{\mathcal{L}_{s}}+i \tilde{\Omega}_{\mathcal{L}_{s}}\right) M\right]^{-1} \tilde{R}_{\mathcal{L}_{s} \emptyset}\left(\left\{\zeta \lambda_{k}+i \tilde{\omega}_{k}\right\} ;\left\{\tilde{\omega}_{k}\right\}\right)|\Sigma\rangle .}
\end{aligned}
$$


This looks very similar to Eq. $(9.12)$ but all terms with $\tilde{\Omega}_{\mathcal{L}_{i}} \neq 0$ have been put into the $\tilde{R}_{\mathcal{L}_{i} \mathcal{L}_{i+1}}\left(\left\{\zeta \lambda_{k}+\right.\right.$ $\left.\left.i \tilde{\omega}_{k}\right\} ;\left\{\tilde{\omega}_{k}\right\}\right)$. Therefore,

$$
\begin{aligned}
\left|\hat{C}_{\mathcal{L}_{\mathrm{i}}}\left(\left\{\zeta \lambda_{k}+i \tilde{\omega}_{k}\right\}\right)\right\rangle= & \sum_{s} \sum_{\substack{\mathcal{L}_{2}, \ldots, \mathcal{L}_{s} \\
\mathcal{L}_{\mathrm{i}}=\mathcal{L}_{1} \supsetneq \mathcal{L}_{2} \supsetneq \cdots \supsetneq \mathcal{L}_{s} \supsetneq \emptyset}}\left[\mathbf{I}-\hat{\Psi}_{0}\left(\zeta \Lambda_{\mathcal{L}_{1}}\right) M\right]^{-1} \tilde{R}_{\mathcal{L}_{1} \mathcal{L}_{2}}\left(\left\{\zeta \lambda_{k}+i \tilde{\omega}_{k}\right\} ;\left\{\tilde{\omega}_{k}\right\}\right) \\
& {\left[\mathbf{I}-\hat{\Psi}_{0}\left(\zeta \Lambda_{\mathcal{L}_{2}}\right) M\right]^{-1} \cdots\left[\mathbf{I}-\hat{\Psi}_{0}\left(\zeta \Lambda_{\mathcal{L}_{s}}\right) M\right]^{-1} \tilde{R}_{\mathcal{L}_{s} \emptyset}\left(\left\{\zeta \lambda_{k}+i \tilde{\omega}_{k}\right\} ;\left\{\tilde{\omega}_{k}\right\}\right)|\Sigma\rangle . }
\end{aligned}
$$

On the other hand, no term in $\tilde{R}_{\mathcal{L}_{1} \mathcal{L}_{2}}\left(\left\{\zeta \lambda_{k}+i \tilde{\omega}_{k}\right\} ;\left\{\tilde{\omega}_{k}\right\}\right)$ is singular, i.e.,

$$
\lim _{\zeta \rightarrow 0} \tilde{R}_{\mathcal{L}_{1} \mathcal{L}_{2}}\left(\left\{\zeta \lambda_{k}+i \tilde{\omega}_{k}\right\} ;\left\{\tilde{\omega}_{k}\right\}\right)=\tilde{R}_{\mathcal{L}_{1} \mathcal{L}_{2}}\left(\left\{i \tilde{\omega}_{k}\right\} ;\left\{\tilde{\omega}_{k}\right\}\right)
$$

\subsection{Long-Time Limit}

With these definition, we can start calculating the last line of Eq. (9.9)

$$
\begin{aligned}
& \lim _{\zeta \rightarrow 0} \zeta^{p \alpha}\left|\hat{C}_{\{1, \ldots, 2 p\}}\left(\left\{\zeta \lambda_{k}+i \tilde{\omega}_{k}\right\}\right)\right\rangle \\
& =\lim _{\zeta \rightarrow 0} \zeta^{p \alpha} \sum_{s} \sum_{\substack{\mathcal{L}_{2}, \ldots, \mathcal{L}_{s} \\
\{1, \ldots, 2 p\}=\mathcal{L}_{1} \supsetneq \mathcal{L}_{2} \supsetneq \ldots \supsetneq \mathcal{L}_{s} \supsetneq \emptyset}}\left[\mathbf{I}-\hat{\Psi}_{0}\left(\zeta \Lambda_{\mathcal{L}_{1}}\right) M\right]^{-1} \tilde{R}_{\mathcal{L}_{1} \mathcal{L}_{2}}\left(\left\{\zeta \lambda_{k}+i \tilde{\omega}_{k}\right\} ;\left\{\tilde{\omega}_{k}\right\}\right) \\
& {\left[\mathbf{I}-\hat{\Psi}_{0}\left(\zeta \Lambda_{\mathcal{L}_{2}}\right) M\right]^{-1} \cdots \tilde{R}_{\mathcal{L}_{s-1} \mathcal{L}_{s}}\left(\left\{\zeta \lambda_{k}+i \tilde{\omega}_{k}\right\} ;\left\{\tilde{\omega}_{k}\right\}\right)|\Sigma\rangle} \\
& =\lim _{\zeta \rightarrow 0} \sum_{s} \zeta^{(p-s) \alpha} \sum_{\substack{\mathcal{L}_{2}, \ldots, \mathcal{L}_{s} \\
\{1, \ldots, 2 p\}=\mathcal{L}_{1} \supsetneq \mathcal{L}_{2} \supsetneq \cdots \supsetneq \mathcal{L}_{s} \supsetneq \emptyset}} \frac{|\Sigma\rangle\left\langle\mathrm{eq}_{\mathrm{M}}\right|}{\bar{\tau}^{\alpha} \Lambda_{\mathcal{L}_{1}}^{\alpha}} \tilde{R}_{\mathcal{L}_{1} \mathcal{L}_{2}}\left(\left\{i \tilde{\omega}_{k}\right\} ;\left\{\tilde{\omega}_{k}\right\}\right) \frac{|\Sigma\rangle\left\langle\mathrm{eq}_{\mathrm{M}}\right|}{\bar{\tau}^{\alpha} \Lambda_{\mathcal{L}_{2}}^{\alpha}}
\end{aligned}
$$

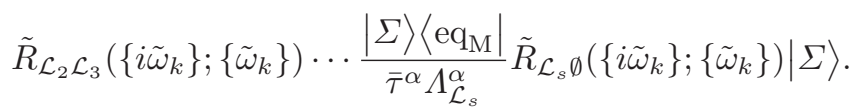

Since all frequencies $\tilde{\omega}_{i} \neq 0$, we know that the conditions $\tilde{\Omega}_{\mathcal{L}_{j}}=0$ and $\tilde{\Omega}_{\mathcal{L}_{j+1}}=0$ can only be fulfilled simultaneously when $\mathcal{L}_{j}$ has at least two elements more than $\mathcal{L}_{j+1}$. Therefore the maximal value of $s$ is $p$. In Eq. (9.23) the scaling variable $\zeta$ appears only in $\zeta^{(p-s) \alpha}$. Any contribution surviving the limit $\zeta \rightarrow 0$ must have $s=p$. Therefore

$$
\begin{aligned}
& \lim _{\zeta \rightarrow 0} \zeta^{p \alpha}\left|\hat{C}_{\{1, \ldots, 2 p\}}\left(\left\{\zeta \lambda_{k}+i \tilde{\omega}_{k}\right\}\right)\right\rangle \\
& =|\Sigma\rangle \sum_{\substack{\mathcal{L}_{2}, \ldots, \mathcal{L}_{p} \\
\{1, \ldots, 2 p\}=\mathcal{L}_{1} \supsetneq \mathcal{L}_{2} \supsetneq \cdots \supsetneq \mathcal{L}_{p} \supsetneq \emptyset}} \frac{1}{\bar{\tau}^{\alpha} \Lambda_{\mathcal{L}_{1}}^{\alpha}}\left\langle\operatorname{eq}\left|\tilde{R}_{\mathcal{L}_{1} \mathcal{L}_{2}}\left(\left\{i \tilde{\omega}_{k}\right\} ;\left\{\tilde{\omega}_{k}\right\}\right)\right| \Sigma\right\rangle \\
& \quad \frac{1}{\bar{\tau}^{\alpha} \Lambda_{\mathcal{L}_{2}}^{\alpha}} \cdots \frac{1}{\bar{\tau}^{\alpha} \Lambda_{\mathcal{L}_{p}}^{\alpha}}\left\langle\mathrm{eq}_{\mathrm{M}}\left|\tilde{R}_{\mathcal{L}_{p} \emptyset}\left(\left\{i \tilde{\omega}_{k}\right\} ;\left\{\tilde{\omega}_{k}\right\}\right)\right| \Sigma\right\rangle .
\end{aligned}
$$

We have a sum over a nested sequence $\{1, \ldots, 2 p\}=\mathcal{L}_{1} \supsetneq \cdots \supsetneq \mathcal{L}_{p} \supsetneq \mathcal{L}_{p+1}=\emptyset$ of $p$ true subsets of $\{1, \ldots, 2 p\}$. Each $\mathcal{L}_{j}$ has at least two elements more than $\mathcal{L}_{j+1}$. There are $p$ such conditions in a set with $2 p$ elements, therefore each $\mathcal{L}_{j}$ contains exactly two elements more than $\mathcal{L}_{j+1}$ which we call $f^{+}(j)$ and $f^{-}(j)$, i.e., $\mathcal{L}_{j}=\mathcal{L}_{j+1} \cup\left\{f^{+}(j), f^{-}(j)\right\}$. As $\tilde{\Omega}_{\mathcal{L}_{j}}=\tilde{\Omega}_{\mathcal{L}_{j+1}}=0$, they must fulfill $\tilde{\omega}_{f^{+}(j)}+\tilde{\omega}_{f^{-}(j)}=0$. The ordering of these two indices is given by the condition $\tilde{\omega}_{f^{+}(j)}>0$ (and correspondingly $\tilde{\omega}_{f^{-}(j)}<0$ ). By definition Eq. (9.5), we have a $g(j)=1 / 2\left(f^{+}(j)+1\right)$ such that $\bar{\omega}_{g(j)}=\tilde{\omega}_{f+(j)}=-\tilde{\omega}_{f-(j)}$. For $j$ running from 1 to $p$ the frequencies $\bar{\omega}_{g(j)}$ take exactly the values of $\bar{\omega}_{1}, \ldots, \bar{\omega}_{p}$ with their exact multiplicity. 
Expanding $\tilde{R}_{\mathcal{L}_{j} \mathcal{L}_{j+1}}\left(\left\{i \tilde{\omega}_{k}\right\} ;\left\{\tilde{\omega}_{k}\right\}\right)$ according to Eq. (9.19) in this case consists of the three nested sequences

$$
\begin{aligned}
& \text { i) } \quad \mathcal{L}_{j} \supsetneq \mathcal{L}_{j+1} \\
& \text { ii) } \quad \mathcal{L}_{j} \supsetneq \mathcal{L}_{j+1}^{+} \subsetneq \mathcal{L}_{j+1} \quad \text { where } \mathcal{L}_{j+1}^{+}=\mathcal{L}_{j+1} \cup\left\{f^{+}(j)\right\} \\
& \text { iii) } \quad \mathcal{L}_{j} \supsetneq \mathcal{L}_{j+1}^{-} \subsetneq \mathcal{L}_{j+1} \quad \text { where } \mathcal{L}_{j+1}^{-}=\mathcal{L}_{j+1} \cup\left\{f^{-}(j)\right\} \text {. }
\end{aligned}
$$

We have $\tilde{\Omega}_{\mathcal{L}_{j}}=\tilde{\Omega}_{\mathcal{L}_{j+1}}=0, \tilde{\Omega}_{\mathcal{L}_{j+1}^{+}}=\tilde{\Omega}_{\mathcal{L}_{j+1}}+\tilde{\omega}_{f+(j)}=\bar{\omega}_{g(j)}$ and $\tilde{\Omega}_{\mathcal{L}_{j+1}^{-}}=\tilde{\Omega}_{\mathcal{L}_{j+1}}+\tilde{\omega}_{f^{-}(j)}=-\bar{\omega}_{g(j)}$.

Using the definition of $\tilde{R}_{\mathcal{L}_{j} \mathcal{L}_{j+1}}\left(\left\{i \tilde{\omega}_{k}\right\} ;\left\{\tilde{\omega}_{k}\right\}\right)$ we calculate in this case using Eqs. (5.8) and (8.15)

$$
\begin{aligned}
\left\langle\mathrm{eq}_{\mathrm{M}}\right| & \tilde{R}_{\mathcal{L}_{j} \mathcal{L}_{j+1}}\left(\left\{i \tilde{\omega}_{k}\right\} ;\left\{\tilde{\omega}_{k}\right\}\right)|\Sigma\rangle \\
= & \left\langle\mathrm{eq}_{\mathrm{M}}\left|\hat{R}_{\mathcal{L}_{j} \mathcal{L}_{j+1}}\left(\left\{i \tilde{\omega}_{k}\right\}\right)\right| \Sigma\right\rangle \\
& +\left\langle\mathrm{eq}_{\mathrm{M}}\left|\hat{R}_{\mathcal{L}_{j} \mathcal{L}_{j+1}^{+}}\left(\left\{i \tilde{\omega}_{k}\right\}\right)\left[\mathbf{I}-\hat{\Psi}_{0}\left(i \tilde{\Omega}_{\mathcal{L}_{j+1}^{+}}\right) M\right]^{-1} \hat{R}_{\mathcal{L}_{j+1}^{+} \mathcal{L}_{j+1}}\left(\left\{i \tilde{\omega}_{k}\right\}\right)\right| \Sigma\right\rangle \\
& +\left\langle\mathrm{eq}_{\mathrm{M}}\left|\hat{R}_{\mathcal{L}_{j} \mathcal{L}_{j+1}^{-}}\left(\left\{i \tilde{\omega}_{k}\right\}\right)\left[\mathbf{I}-\hat{\Psi}_{0}\left(i \tilde{\Omega}_{\mathcal{L}_{j+1}^{-}}\right) M\right]^{-1} \hat{R}_{\mathcal{L}_{j+1}^{-} \mathcal{L}_{j+1}}\left(\left\{i \tilde{\omega}_{k}\right\}\right)\right| \Sigma\right\rangle \\
= & \frac{1}{\bar{\omega}_{g(j)}^{2}}\left\langle\mathrm{eq}_{\mathrm{M}}\left|2 \hat{\Psi}_{2}(0)-\hat{\Psi}_{2}\left(i \bar{\omega}_{g(j)}\right)-\hat{\Psi}_{2}\left(-i \bar{\omega}_{g(j)}\right)\right| \Sigma\right\rangle \\
& -\frac{1}{\bar{\omega}_{g(j)}^{2}}\left\langle\mathrm{eq}_{\mathrm{M}}\right|\left(\hat{\Psi}_{1}(0)-\hat{\Psi}_{1}\left(i \bar{\omega}_{g(j)}\right) M\left[\mathbf{I}-\hat{\Psi}_{0}\left(i \bar{\omega}_{g(j)}\right) M\right]^{-1}\left(\hat{\Psi}_{1}(0)-\hat{\Psi}_{1}\left(i \bar{\omega}_{g(j)}\right)|\Sigma\rangle\right.\right. \\
& -\frac{1}{\bar{\omega}_{g(j)}^{2}}\left\langle\mathrm{eq}_{\mathrm{M}}\right|\left(\hat{\Psi}_{1}(0)-\hat{\Psi}_{1}\left(-i \bar{\omega}_{g(j)}\right) M\left[\mathbf{I}-\hat{\Psi}_{0}\left(-i \bar{\omega}_{g(j)}\right) M\right]^{-1}\left(\hat{\Psi}_{1}(0)-\hat{\Psi}_{1}\left(-i \bar{\omega}_{g(j)}\right)|\Sigma\rangle\right.\right. \\
= & \Gamma(1+\alpha) \bar{\tau}^{\alpha} \bar{S}_{\alpha}\left(\bar{\omega}_{g(j)}\right) .
\end{aligned}
$$

Using these results in Eqs. (9.9) and (9.24) gives (using $\prod_{j=1}^{p} \bar{S}_{\alpha}\left(\bar{\omega}_{g(j)}\right)=\prod_{j=1}^{p} \bar{S}_{\alpha}\left(\bar{\omega}_{j}\right)$ )

$$
\begin{aligned}
& \lim _{\zeta \rightarrow 0} \mathrm{~L}\left[\zeta^{p \alpha}\left\langle F_{T_{1} / \zeta}\left(-\tilde{\omega}_{1}\right) \cdots F_{T_{2 p} / \zeta}\left(-\tilde{\omega}_{2 p}\right)\right\rangle_{\langle\text {init }}\right] \\
& =\lim _{\zeta \rightarrow 0} \frac{\zeta^{p \alpha}}{\lambda_{1} \cdots \lambda_{2 p}}\left\langle\text { init } \mid \hat{C}_{\{1, \ldots, 2 p\}}\left(\left\{\zeta \lambda_{k}+i \tilde{\omega}_{k}\right\}\right)\right\rangle . \\
& =\Gamma(1+\alpha)^{p} \prod_{j=1}^{p} \bar{S}_{\alpha}\left(\bar{\omega}_{j}\right) \sum_{\substack{\mathcal{L}_{2}, \ldots, \mathcal{L}_{p} \\
\{1, \ldots, 2 p\}=\mathcal{L}_{1} \supsetneq \mathcal{L}_{2} \supsetneq \cdots \mathcal{L}_{p} \supsetneq \emptyset \\
\tilde{\Omega}_{\mathcal{L}_{2}}=\tilde{\Omega}_{\mathcal{L}_{2}=\cdots=\tilde{\Omega}_{\mathcal{L}_{p}}=0}}} \frac{1}{\lambda_{1} \cdots \lambda_{2 p}} \frac{1}{\Lambda_{\mathcal{L}_{1}}^{\alpha} \cdots \Lambda_{\mathcal{L}_{p}}^{\alpha}} .
\end{aligned}
$$

Here, we have to explicitly state the condition $\tilde{\Omega}_{\mathcal{L}_{2}}=\tilde{\Omega}_{\mathcal{L}_{2}}=\cdots=\tilde{\Omega}_{\mathcal{L}_{p}}=0$ as this is not enforced by the $\tilde{R}_{\mathcal{L}_{j} \mathcal{L}_{j+1}}\left(\left\{i \tilde{\omega}_{k}\right\} ;\left\{\tilde{\omega}_{k}\right\}\right)$ any more.

The next step is to invert the Laplace transform

$$
\mathrm{L}^{-1}\left[\frac{1}{\lambda_{1} \cdots \lambda_{2 p}} \frac{1}{\Lambda_{\mathcal{L}_{1}}^{\alpha} \cdots \Lambda_{\mathcal{L}_{p}}^{\alpha}}\right]
$$

at the point $T_{1}=\cdots=T_{2 p}=T$. This calculation is done in Appendix A. Eq. (A.10) reads

$$
\mathrm{L}^{-1}\left[\frac{1}{\lambda_{1} \cdots \lambda_{2 p}} \frac{1}{\Lambda_{\mathcal{L}_{1}}^{\alpha} \cdots \Lambda_{\mathcal{L}_{p}}^{\alpha}}\right]\left(T_{1}=\cdots=T_{2 p}=T\right)=\frac{T^{p \alpha}}{\Gamma(1+p \alpha)}
$$


and therefore

$$
\lim _{\zeta \rightarrow 0} \zeta^{p \alpha}\left\langle F_{T / \zeta}\left(-\tilde{\omega}_{1}\right) \cdots F_{T / \zeta}\left(-\tilde{\omega}_{2 p}\right)\right\rangle_{\langle\text {init }|}=T^{p \alpha} \frac{\Gamma(1+\alpha)^{p}}{\Gamma(1+p \alpha)} \prod_{j=1}^{p} \bar{S}_{\alpha}\left(\bar{\omega}_{j}\right) \sum_{\begin{array}{c}
\mathcal{L}_{2}, \ldots, \mathcal{L}_{p} \\
\{1, \ldots, 2 p\}=\mathcal{L}_{1} \supsetneq \mathcal{L}_{2} \supsetneq \cdots \mathcal{L}_{p} \supsetneq \emptyset \\
\tilde{\Omega}_{\mathcal{L}_{2}}=\tilde{\Omega}_{\mathcal{L}_{2}}=\cdots=\tilde{\Omega}_{\mathcal{L}_{p}}=0
\end{array}} 1
$$

Switching back to the frequencies $\omega_{i}\left(p=q_{1}+\cdots+q_{r}\right)$ which are pairwise different (Eq. (9.3)), we get for long times

$$
\begin{aligned}
& \lim _{T \rightarrow \infty} \frac{\left\langle S_{T}\left(\omega_{1}\right)^{q_{1}} \cdots S_{T}\left(\omega_{r}\right)^{q_{r}}\right\rangle}{\left\langle S_{T}\left(\omega_{1}\right)\right\rangle^{q_{1}} \cdots\left\langle S_{T}\left(\omega_{r}\right)^{q_{r}}\right.}=\frac{\Gamma(1+\alpha)^{p}}{\Gamma(1+p \alpha)} \quad \sum_{\mathcal{L}_{2} \ldots, \mathcal{L}_{p}} 1 . \\
& \begin{array}{c}
\{1, \ldots, 2 p\}=\mathcal{L}_{1} \supsetneq \mathcal{L}_{2} \supsetneq \ldots \supsetneq \mathcal{L}_{p} \supsetneq \emptyset \\
\tilde{\Omega}_{\mathcal{L}_{2}}=\tilde{\Omega}_{\mathcal{L}_{2}}=\cdots=\tilde{\Omega}_{\mathcal{L}_{p}=0}
\end{array}
\end{aligned}
$$

In a last step we have to determine the combinatorial expression

$$
\sum_{\substack{\mathcal{L}_{2}, \ldots, \mathcal{L}_{p} \\\{1, \ldots, 2 p\}=\mathcal{L}_{1} \supsetneq \mathcal{L}_{2} \supsetneq \mathcal{L}_{p} \supsetneq \emptyset \\ \tilde{\Omega}_{\mathcal{L}_{2}}=\tilde{\Omega}_{\mathcal{L}_{2}=\cdots=\tilde{\Omega}_{\mathcal{L}_{p}}=0}}} 1
$$

which counts the number of nested sequences of subsets $\{1, \ldots, 2 p\}=\mathcal{L}_{1} \supsetneq \mathcal{L}_{2} \supsetneq \cdots \supsetneq \mathcal{L}_{p} \supsetneq \emptyset$ with the condition $\tilde{\Omega}_{\mathcal{L}_{1}}=\tilde{\Omega}_{\mathcal{L}_{2}}=\cdots=\tilde{\Omega}_{\mathcal{L}_{p}}=0$.

We have already argued, that

$$
\mathcal{L}_{j}=\mathcal{L}_{j+1} \cup\left\{f^{+}(j), f^{-}(j)\right\}
$$

where $f^{+}(j)$ and $f^{-}(j)$ take value in $1, \ldots, 2 p$ with $\tilde{\omega}_{f^{+}(j)}+\tilde{\omega}_{f^{-}(j)}=0$. By demanding $\tilde{\omega}_{f+(j)}>0$ (resp. $\left.\tilde{\omega}_{f^{+}(j)}<0\right)$ the functions are unique. Therefore, we have to count how many functions $f^{+}(j)$ and $f^{-}(j)$ exist with $\tilde{\omega}_{f+(j)}>0$ and $\tilde{\omega}_{f^{-}(j)}=-\tilde{\omega}_{f+(j)}$. For $f^{+}(j)$ we can take any permutation such that $\tilde{\omega}_{f^{+}(j)}$ is positive. Therefore, we have $p$ ! different functions. Once $f^{+}(j)$ is fixed we can only permute the values of $f^{-}(j)$ if $\tilde{\omega}_{f^{-}(j)}$ does not change. For a fixed $f^{+}(j)$ we have therefore $q_{1} ! \cdots q_{r}$ ! possibilities for $f^{-}(j)$. This gives

$$
\sum_{\substack{\mathcal{L}_{2}, \ldots, \mathcal{L}_{p} \\\{1, \ldots, 2 p\}=\mathcal{L}_{1} \supsetneq \mathcal{L}_{2} \supsetneq \tilde{L}_{2} \supsetneq \mathcal{L}_{p} \supsetneq \emptyset \\ \tilde{\Omega}_{\mathcal{L}_{2}}=\tilde{\Omega}_{\mathcal{L}_{2}=\cdots=\tilde{\Omega}_{\mathcal{L}_{p}}=0}}} 1=p ! q_{1} ! \cdots q_{r} !
$$

Using this in Eq. (9.31) gives $\left(p=q_{1}+\cdots+q_{r}\right)$

$$
\lim _{T \rightarrow \infty} \frac{\left\langle S_{T}\left(\omega_{1}\right)^{q_{1}} \cdots S_{T}\left(\omega_{r}\right)^{q_{r}}\right\rangle}{\left\langle S_{T}\left(\omega_{1}\right)\right\rangle^{q_{1}} \cdots\left\langle S_{T}\left(\omega_{r}\right\rangle^{q_{r}}\right.}=\frac{p ! \Gamma(1+\alpha)^{p}}{\Gamma(1+p \alpha)} q_{1} ! \cdots q_{r} !
$$

or alternatively written for large $T$

$$
\lim _{T \rightarrow \infty} T^{p(1-\alpha)}\left\langle S_{T}\left(\omega_{1}\right)^{q_{1}} \cdots S_{T}\left(\omega_{r}\right)^{q_{r}}\right\rangle \simeq \frac{p ! \Gamma(1+\alpha)^{p}}{\Gamma(1+p \alpha)} \prod_{j=1}^{r} q_{j} ! \bar{S}_{\alpha}\left(\omega_{j}\right)^{q_{j}}
$$

\subsection{Distribution of the Spectral Observables}

We can now show our main result for the correlation of the spectral density by using the method of moments [11]. We want to show the convergence in distribution for large $T$

$$
\lim _{T \rightarrow \infty} T^{1-\alpha}\left(S_{T}\left(\omega_{1}\right), \ldots, S_{T}\left(\omega_{r}\right)\right)=Y_{\alpha}\left(E_{1} \bar{S}_{\alpha}\left(\omega_{1}\right), \ldots, E_{r} \bar{S}_{\alpha}\left(\omega_{r}\right)\right)
$$


where $Y_{\alpha}, E_{1}, \ldots, E_{r}$ are independent random variables. The random variable $Y_{\alpha}$ is of Mittag-Leffler type which can be defined by its moments $\left\langle Y_{\alpha}^{n}\right\rangle=\frac{n ! \Gamma(1+\alpha)^{n}}{\Gamma(1+n \alpha)}$. The random variable $E_{i}$ are exponentially distributed with moments $\left\langle E_{i}^{n}\right\rangle=n$ !. Calculating a moment using the right side of Eq. (9.37) gives

$$
\begin{aligned}
\lim _{T \rightarrow \infty}\left\langle\left(T^{1-\alpha} S_{T}\left(\omega_{1}\right)^{q_{1}}\right)^{q_{1}} \cdots\left(T^{1-\alpha} S_{T}\left(\omega_{1}\right)^{q_{r}}\right)^{q_{r}}\right\rangle & =\lim _{T \rightarrow \infty} T^{p(1-\alpha)}\left\langle S_{T}\left(\omega_{1}\right)^{q_{1}} \cdots S_{T}\left(\omega_{r}\right)^{q_{r}}\right\rangle \\
& =\left\langle Y_{\alpha}^{p}\right\rangle \prod_{j=1}^{r}\left\langle E_{j}^{q_{j}} \bar{S}_{\alpha}\left(\omega_{j}\right)^{q_{j}}\right\rangle \\
& =\frac{p ! \Gamma(1+\alpha)^{p}}{\Gamma(1+p \alpha)} \prod_{j=1}^{r} q_{j} ! \bar{S}_{\alpha}\left(\omega_{j}\right)^{q_{j}} .
\end{aligned}
$$

As these are the same moments as determined in Eq. (9.36), we can conclude the convergence in distribution described by Eq. (9.37).

\section{The Spectrum of an Aged Process}

Sometimes, it is also interesting to consider the determination of a process which has aged a time $t_{\mathrm{a}}$ before the measurement starts. We will develop the argument closely to argument in Section 9 - we will also rely heavily on the derivations done there.

First we define the aged Fourier transform of the time series

$$
F_{T}^{\mathrm{aged}}\left(\omega ; t_{\mathrm{a}}\right)=\int_{0}^{T} \mathrm{~d} t \exp (i \omega t) X\left(t+t_{\mathrm{a}}\right)
$$

and the corresponding periodogram estimator for the spectrum

$$
S_{T}^{\text {aged }}\left(\omega ; t_{\mathrm{a}}\right)=\frac{1}{T} F_{T}^{\text {aged }}\left(\omega ; t_{\mathrm{a}}\right) F_{T}^{\text {aged }}\left(-\omega ; t_{\mathrm{a}}\right) .
$$

We are interested in the behavior of

$$
\left\langle S_{T}^{\text {aged }}\left(\omega_{1} ; t_{\mathrm{a}}\right)^{q_{1}} \cdots S_{T}^{\text {aged }}\left(\omega_{r} ; t_{\mathrm{a}}\right)^{q_{r}}\right\rangle_{\langle\text {init }|}
$$

for large $T$ and $t_{\mathrm{a}}$ where the $\omega_{i}>0$ are pairwise disjoint. Following Eq. (9.3) we define the $\bar{\omega}_{1}, \ldots, \bar{\omega}_{p}$ with $p=q_{1}+\cdots+q_{r}$

$$
\left\langle S_{T}^{\text {aged }}\left(\bar{\omega}_{1} ; t_{\mathrm{a}}\right) \cdots S_{T}^{\text {aged }}\left(\bar{\omega}_{p} ; t_{\mathrm{a}}\right)\right\rangle_{\langle\text {init }|}=\left\langle S_{T}^{\text {aged }}\left(\omega_{1} ; t_{\mathrm{a}}\right)^{q_{1}} \cdots S_{T}^{\text {aged }}\left(\omega_{r} ; t_{\mathrm{a}}\right)^{q_{r}}\right\rangle_{\langle\text {init }|} .
$$

We also continue to use the definitions Eq. (9.5) for $\tilde{\omega}_{i}$ and Eq. (9.6) for $\tilde{\Omega}_{\mathcal{L}}$. Following Eq. (9.7) we introduce several time points

$$
T^{p}\left\langle S_{T}^{\text {aged }}\left(\bar{\omega}_{1} ; t_{\mathrm{a}}\right) S_{T}^{\text {aged }}\left(\bar{\omega}_{p} ; t_{\mathrm{a}}\right)\right\rangle_{\langle\text {init }|}=\left.\left\langle F_{T_{1}}^{\text {aged }}\left(-\tilde{\omega}_{1} ; t_{\mathrm{a}}\right) \cdots F_{T_{2 p}}^{\text {aged }}\left(-\tilde{\omega}_{2 p} ; t_{\mathrm{a}}\right)\right\rangle_{\langle\text {init }|}\right|_{T_{1}=\cdots=T_{2 p}=T} .
$$

\subsection{Constant Aged Time}

In a first step, we are interested in the limit of the measurement time $T$ going to infinity while the aged time $t_{\mathrm{a}}$ stays constant. For this we take the limit

$$
\left.\lim _{\zeta \rightarrow 0} \zeta^{p \alpha}\left\langle F_{T_{1} / \zeta}^{\text {aged }}\left(-\tilde{\omega}_{1} ; t_{\mathrm{a}}\right) \cdots F_{T_{2 p} / \zeta}^{\text {aged }}\left(-\tilde{\omega}_{2 p} ; t_{\mathrm{a}}\right)\right\rangle_{\langle\text {init }|}\right|_{T_{1}=\cdots=T_{2 p}=T} .
$$


with the Laplace transform (see Eq. (9.9), we additionally take the Laplace transform of $t_{\mathrm{a}}$ with Laplace partner $s_{\mathrm{a}}$ )

$$
\begin{aligned}
& \mathrm{L}\left[\zeta ^ { p \alpha } \left\langleF _ { T _ { 1 } / \zeta } ^ { \text { aged } } \left(-\tilde{\omega}_{1} ;\right.\right.\right.\left.\left.\left.t_{\mathrm{a}}\right) \cdots F_{T_{2 p} / \zeta}^{\text {aged }}\left(-\tilde{\omega}_{2 p} ; t_{\mathrm{a}}\right)\right\rangle\langle\text { init }|\right] \\
&=\zeta^{p \alpha} \int_{0}^{\infty} \mathrm{d} t_{\mathrm{a}} e^{-s_{\mathrm{a}} t_{\mathrm{a}}} \int_{0}^{\infty} \mathrm{d} T_{1} \cdots \int_{0}^{\infty} \mathrm{d} T_{2 p} e^{-\lambda_{1} T_{1}-\cdots-\lambda_{2 p} T_{2 p}} \\
& \times \int_{0}^{T_{1} / \zeta} \mathrm{d} t_{1} \cdots \int_{0}^{T_{2 p} / \zeta} \mathrm{d} t_{2 p} e^{-i\left(\tilde{\omega}_{1} t_{1}+\cdots+\tilde{\omega}_{2 p} t_{2 p}\right)}\left\langle X\left(t_{1}+t_{\mathrm{a}}\right) \cdots X\left(t_{2 p}+t_{\mathrm{a}}\right)\right\rangle_{\langle\text {init }|} \\
&= \frac{\zeta^{p \alpha}}{\lambda_{1} \cdots \lambda_{2 p}}\left\langle\text { init } \mid \hat{C}_{\{1, \ldots, 2 p\}}^{\mathrm{aged}}\left(\left\{\zeta \lambda_{k}+i \tilde{\omega}_{k}\right\} ; s_{\mathrm{a}}\right)\right\rangle .
\end{aligned}
$$

The correlation on the right hand side can be reduced to known quantities by Eq. (6.4)

$$
\left|\hat{C}_{\{1, \ldots, 2 p\}}^{\mathrm{aged}}\left(\left\{\zeta \lambda_{k}+i \tilde{\omega}_{k}\right\} ; s_{\mathrm{a}}\right)\right\rangle=\sum_{\mathcal{L}_{\mathrm{f}}:\{1, \ldots, 2 p\} \supseteq \mathcal{L}_{\mathrm{f}}} \hat{R}_{\{1, \ldots, 2 p\} \mathcal{L}_{\mathrm{f}}}^{\mathrm{fw}}\left(\left\{\zeta \lambda_{k}+i \tilde{\omega}_{k}\right\} ; s_{\mathrm{a}}\right)\left|\hat{C}_{\mathcal{L}_{\mathrm{f}}}\left(\left\{\zeta \lambda_{k}+i \tilde{\omega}_{k}\right\}\right)\right\rangle
$$

with the definitions Eq. (6.5)

$$
\hat{R}_{\mathcal{L}_{\mathrm{i}} \mathcal{L}_{\mathrm{f}}}^{\mathrm{fw}}\left(\left\{\lambda_{k}\right\} ; s_{\mathrm{a}}\right)=\frac{1}{\prod_{k \in \mathcal{L}_{\mathrm{i}} \backslash \mathcal{L}_{\mathrm{f}}} \lambda_{k}} \sum_{\mathcal{J}: \mathcal{L}_{\mathrm{i}} \supseteq \mathcal{J} \supseteq \mathcal{L}_{\mathrm{f}}}(-1)^{\left|\mathcal{J} \backslash \mathcal{L}_{\mathrm{f}}\right|} \hat{\Psi}_{\left|\mathcal{L}_{\mathrm{i}}^{\mathrm{fw}} \backslash \mathcal{L}_{\mathrm{f}}\right|}\left(\Lambda_{\mathcal{J}} ; s_{\mathrm{a}}\right) M
$$

and Eq. (4.11)

$$
\hat{\Psi}_{n}^{\mathrm{fw}}(\lambda ; s)=\frac{1}{s_{\mathrm{a}}-\lambda}\left[\mathbf{I}-\hat{\Psi}_{0}\left(s_{\mathrm{a}}\right) M\right]^{-1}\left(\hat{\Psi}_{n}(\lambda)-\hat{\Psi}_{n}\left(s_{\mathrm{a}}\right)\right) .
$$

Therefore the term $\hat{R}_{\{1, \ldots, 2 p\} \mathcal{L}_{\mathrm{f}}}^{\mathrm{fw}}\left(\left\{\zeta \lambda_{k}+i \tilde{\omega}_{k}\right\} ; s_{\mathrm{a}}\right)$ is not singular

$$
\lim _{\zeta \rightarrow 0} \hat{R}_{\{1, \ldots, 2 p\} \mathcal{L}_{\mathrm{f}}}^{\mathrm{fw}}\left(\left\{\zeta \lambda_{k}+i \tilde{\omega}_{k}\right\} ; s_{\mathrm{a}}\right)=\hat{R}_{\{1, \ldots, 2 p\} \mathcal{L}_{\mathrm{f}}}^{\mathrm{fw}}\left(\left\{i \tilde{\omega}_{k}\right\} ; s_{\mathrm{a}}\right)
$$

and

$$
\lim _{\zeta \rightarrow 0} \zeta^{p \alpha}\left|\hat{C}_{\{1, \ldots, 2 p\}}^{\mathrm{aged}}\left(\left\{\zeta \lambda_{k}+i \tilde{\omega}_{k}\right\} ; s_{\mathrm{a}}\right)\right\rangle=\sum_{\mathcal{L}_{\mathrm{f}}:\{1, \ldots, 2 p\} \supseteq \mathcal{L}_{\mathrm{f}}} \hat{R}_{\{1, \ldots, 2 p\} \mathcal{L}_{\mathrm{f}}}^{\mathrm{fw}}\left(\left\{i \tilde{\omega}_{k}\right\} ; s_{\mathrm{a}}\right) \lim _{\zeta \rightarrow 0} \zeta^{p \alpha}\left|\hat{C}_{\mathcal{L}_{\mathrm{f}}}\left(\left\{\zeta \lambda_{k}+i \tilde{\omega}_{k}\right\}\right)\right\rangle .
$$

We have to determine the $\operatorname{limit}_{\lim _{\zeta \rightarrow 0}} \zeta^{p \alpha}\left|\hat{C}_{\mathcal{L}_{\mathrm{f}}}\left(\left\{\zeta \lambda_{k}+i \tilde{\omega}_{k}\right\}\right)\right\rangle$. For $\mathcal{L}_{\mathrm{f}}=\{1, \ldots, 2 p\}$ we have calculated in Section 9 (Eqs. (9.24) and (9.26))

$$
\begin{aligned}
& \lim _{\zeta \rightarrow 0} \zeta^{p \alpha}\left|\hat{C}_{\{1, \ldots, 2 p\}}\left(\left\{\zeta \lambda_{k}+i \tilde{\omega}_{k}\right\}\right)\right\rangle \\
& =|\Sigma\rangle \Gamma(1+\alpha)^{p} \prod_{j=1}^{p} \bar{S}_{\alpha}\left(\bar{\omega}_{j}\right) \sum_{\substack{\mathcal{L}_{2}, \ldots, \mathcal{L}_{p} \\
\{1, \ldots, 2 p\}=\mathcal{L}_{1} \supsetneq \mathcal{L}_{2} \supsetneq \cdots \supsetneq \mathcal{L}_{p} \supsetneq \emptyset \\
\tilde{\Omega}_{\mathcal{L}_{2}}=\tilde{\Omega}_{\mathcal{L}_{2}=\cdots=\tilde{\Omega}_{\mathcal{L}_{p}}=0}}} \frac{1}{\Lambda_{\mathcal{L}_{1}}^{\alpha} \cdots \Lambda_{\mathcal{L}_{p}}^{\alpha}} .
\end{aligned}
$$

This leaves us with the cases $\mathcal{L}_{\mathrm{f}} \subsetneq\{1, \ldots, 2 p\}$. In this case we get from Eq. (9.20) (with the knowledge that $\tilde{\Omega}_{\mathcal{L}_{2}}=\cdots=\tilde{\Omega}_{\mathcal{L}_{s}}=0$ )

$$
\begin{aligned}
\left|\hat{C}_{\mathcal{L}_{\mathrm{f}}}\left(\left\{\zeta \lambda_{k}+i \tilde{\omega}_{k}\right\}\right)\right\rangle= & \sum_{s} \sum_{\substack{\mathcal{L}_{\mathcal{f}}, \ldots, \mathcal{L}_{s} \\
\mathcal{L}_{\mathrm{f}}=\mathcal{L}_{1} \supsetneq \mathcal{L}_{2} \supsetneq \cdots \supsetneq \mathcal{L}_{s} \supsetneq \emptyset}}\left[\mathbf{I}-\hat{\Psi}_{0}\left(\zeta \Lambda_{\mathcal{L}_{1}}+i \tilde{\Omega}_{\mathcal{L}_{1}}\right) M\right]^{-1} \tilde{R}_{\mathcal{L}_{1} \mathcal{L}_{2}}\left(\left\{\zeta \lambda_{k}+i \tilde{\omega}_{k}\right\} ;\left\{\tilde{\omega}_{k}\right\}\right) \\
& {\left[\mathbf{I}-\hat{\Psi}_{0}\left(\zeta \Lambda_{\mathcal{L}_{2}}\right) M\right]^{-1} \cdots\left[\mathbf{I}-\hat{\Psi}_{0}\left(\zeta \Lambda_{\mathcal{L}_{s}}\right) M\right]^{-1} \tilde{R}_{\mathcal{L}_{s} \emptyset}\left(\left\{\zeta \lambda_{k}+i \tilde{\omega}_{k}\right\} ;\left\{\tilde{\omega}_{k}\right\}\right)|\Sigma\rangle . }
\end{aligned}
$$


The only terms which can become singular for $\zeta \rightarrow 0$ are the $\left[\mathbf{I}-\hat{\Psi}_{0}\left(\zeta \Lambda_{\mathcal{L}}\right) M\right]^{-1}$ terms which will contribute with $\zeta^{-\alpha}$. In section 9 we have shown that $\mathcal{L}_{j}$ must have at least two elements more than $\mathcal{L}_{j+1}$. But $\mathcal{L}_{\mathrm{f}}$ can have at most $2 p-1$ elements and we have at most $p-1$ singular terms, i.e.,

$$
\zeta^{p \alpha}\left|\hat{C}_{\mathcal{L}_{\mathrm{f}}}\left(\left\{\zeta \lambda_{k}+i \tilde{\omega}_{k}\right\}\right)\right\rangle=\mathrm{O}\left(\zeta^{\alpha}\right) \text { for } \mathcal{L}_{\mathrm{f}} \subsetneq\{1, \ldots, 2 p\}
$$

Therefore only the term with $\mathcal{L}_{\mathrm{f}}=\{1, \ldots, 2 p\}$ survives in Eq. (10.12):

$$
\begin{aligned}
& \lim _{\zeta \rightarrow 0} \zeta^{p \alpha}\left|\hat{C}_{\{1, \ldots, 2 p\}}^{\mathrm{aged}}\left(\left\{\zeta \lambda_{k}+i \tilde{\omega}_{k}\right\} ; s_{\mathrm{a}}\right)\right\rangle=\hat{R}_{\{1, \ldots, 2 p\}\{1, \ldots, 2 p\}}^{\mathrm{fw}}\left(\left\{i \tilde{\omega}_{k}\right\} ; s_{\mathrm{a}}\right) \lim _{\zeta \rightarrow 0} \zeta^{p \alpha}\left|\hat{C}_{\{1, \ldots, 2 p\}}\left(\left\{\zeta \lambda_{k}+i \tilde{\omega}_{k}\right\}\right)\right\rangle \\
& =\hat{\Psi}_{0}^{\mathrm{fw}}\left(0 ; s_{\mathrm{a}}\right) \lim _{\zeta \rightarrow 0} \zeta^{p \alpha}\left|\hat{C}_{\{1, \ldots, 2 p\}}\left(\left\{\zeta \lambda_{k}+i \tilde{\omega}_{k}\right\}\right)\right\rangle \\
& =\frac{1}{s_{\mathrm{a}}}\left[\mathbf{I}-\hat{\Psi}_{0}\left(s_{\mathrm{a}}\right) M\right]^{-1}\left(\hat{\Psi}_{0}(0)-\hat{\Psi}_{0}\left(s_{\mathrm{a}}\right)\right)|\Sigma\rangle \\
& \times \Gamma(1+\alpha)^{p} \prod_{j=1}^{p} \bar{S}_{\alpha}\left(\bar{\omega}_{j}\right) \quad \sum_{\mathcal{L}_{2}, \ldots, \mathcal{L}_{p}} \quad \frac{1}{\Lambda_{\mathcal{L}_{1}}^{\alpha} \cdots \Lambda_{\mathcal{L}_{p}}^{\alpha}} \\
& \begin{aligned}
&\{1, \ldots, 2 p\}=\mathcal{L}_{2}, \ldots, \mathcal{L}_{p} \supsetneq \ldots \supsetneq \mathcal{L}_{2} \supsetneq \emptyset \emptyset \\
& \tilde{\Omega}_{\mathcal{L}_{2}}=\tilde{\Omega}_{\mathcal{L}_{2}}=\cdots=\tilde{\Omega}_{\mathcal{L}_{p}}=0
\end{aligned}
\end{aligned}
$$

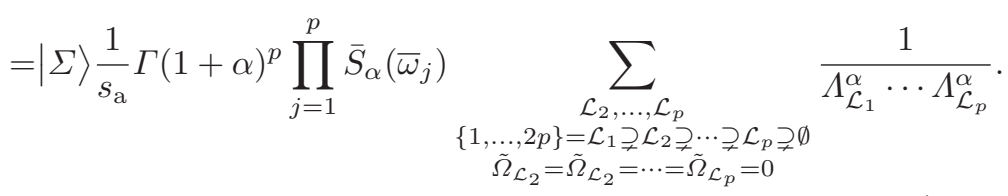

The inverse Laplace transform of $1 / s_{\mathrm{a}}$ is just the constant function with value 1 . The other terms are exactly the ones obtained in Eq. (9.27) without aging. Therefore, in this limit any aging before the measurement $t_{\mathrm{a}}$ does not play a role. This can also be understood heuristically: the aging affects only the first epoch. Since we are observing infinitely many epochs when the measurement time goes to infinity, the first one can be neglected.

\subsection{Aged Time of Order of Observation Time}

In a second step, we are therefore considering the case that the aged time is of the same order of magnitude as the measurement time. This is done by scaling the aged time $t_{\mathrm{a}}$ in the same way as the measurement time

$$
\left.\lim _{\zeta \rightarrow 0} \zeta^{p \alpha}\left\langle F_{T_{1} / \zeta}^{\mathrm{aged}}\left(-\tilde{\omega}_{1} ; t_{\mathrm{a}} / \zeta\right) \cdots F_{T_{2 p} / \zeta}^{\mathrm{aged}}\left(-\tilde{\omega}_{2 p} ; t_{\mathrm{a}} / \zeta\right)\right\rangle_{\langle\text {init }|}\right|_{T_{1}=\cdots=T_{2 p}=T}
$$

The Laplace transform is

$$
\begin{aligned}
& \mathrm{L}\left[\zeta ^ { p \alpha } \left\langleF_{T_{1} / \zeta}^{\text {aged }}\left(-\tilde{\omega}_{1} ; t_{\mathrm{a}} / \zeta\right)\right.\right.\left.\left.\cdots F_{T_{2 p} / \zeta}^{\mathrm{aged}}\left(-\tilde{\omega}_{2 p} ; t_{\mathrm{a}} / \zeta\right)\right\rangle\langle\text { init }|\right] \\
&=\zeta^{p \alpha} \int_{0}^{\infty} \mathrm{d} t_{\mathrm{a}} e^{-s_{\mathrm{a}} t_{\mathrm{a}}} \int_{0}^{\infty} \mathrm{d} T_{1} \cdots \int_{0}^{\infty} \mathrm{d} T_{2 p} e^{-\lambda_{1} T_{1}-\cdots-\lambda_{2 p} T_{2 p}} \\
& \times \int_{0}^{T_{1} / \zeta} \mathrm{d} t_{1} \cdots \int_{0}^{T_{2 p} / \zeta} \mathrm{d} t_{2 p} e^{-i\left(\tilde{\omega}_{1} t_{1}+\cdots+\tilde{\omega}_{2 p} t_{2 p}\right)}\left\langle X\left(t_{1}+t_{\mathrm{a}} / \zeta\right) \cdots X\left(t_{2 p}+t_{\mathrm{a}} / \zeta\right)\right\rangle_{\langle\text {init }|} \\
&= \frac{\zeta^{p \alpha+1}}{\lambda_{1} \cdots \lambda_{2 p}}\left\langle\operatorname{init} \mid \hat{C}_{\{1, \ldots, 2 p\}}^{\mathrm{aged}}\left(\left\{\zeta \lambda_{k}+i \tilde{\omega}_{k}\right\} ; \zeta s_{\mathrm{a}}\right)\right\rangle .
\end{aligned}
$$


We have analogously to Eq. (10.12)

$\lim _{\zeta \rightarrow 0} \zeta^{p \alpha+1}\left|\hat{C}_{\{1, \ldots, 2 p\}}^{\text {aged }}\left(\left\{\zeta \lambda_{k}+i \tilde{\omega}_{k}\right\}, s_{\mathrm{a}}\right)\right\rangle=\lim _{\zeta \rightarrow 0} \sum_{\mathcal{L}_{\mathrm{f}}:\{1, \ldots, 2 p\} \supseteq \mathcal{L}_{\mathrm{f}}} \zeta \hat{R}_{\{1, \ldots, 2 p\} \mathcal{L}_{\mathrm{f}}}^{\mathrm{fw}}\left(\left\{\zeta \lambda_{k}+i \tilde{\omega}_{k}\right\}, \zeta s_{\mathrm{a}}\right) \zeta^{p \alpha}\left|\hat{C}_{\mathcal{L}_{\mathrm{f}}}\left(\left\{\zeta \lambda_{k}+i \tilde{\omega}_{k}\right\}\right)\right\rangle$.

The terms with $\mathcal{L}_{\mathrm{f}} \subsetneq\{1, \ldots, 2 p\}$ are $\mathrm{O}\left(\zeta^{\alpha}\right)$ according to Eq. (10.15). We calculate from Eq. (4.11)

$$
\begin{aligned}
& \lim _{\zeta \rightarrow 0} \zeta^{1+\alpha} \hat{\Psi}_{n}^{\mathrm{fw}}\left(\zeta \Lambda_{\mathcal{J}}+i \tilde{\Omega}_{\mathcal{J}} ; \zeta s_{\mathrm{a}}\right) \\
& =\lim _{\zeta \rightarrow 0} \zeta^{\alpha}\left[\mathbf{I}-\hat{\Psi}_{0}\left(\zeta s_{\mathrm{a}}\right) M\right]^{-1} \frac{\zeta}{\zeta s_{\mathrm{a}}-\zeta \Lambda_{\mathcal{J}}-i \tilde{\Omega}_{\mathcal{J}}}\left(\hat{\Psi}_{n}\left(\zeta \Lambda_{\mathcal{J}}+i \tilde{\Omega}_{\mathcal{J}}\right)-\hat{\Psi}_{n}\left(\zeta s_{\mathrm{a}}\right)\right) \\
& =\frac{|\Sigma\rangle\left\langle\mathrm{eq}_{\mathrm{M}}\right|}{\bar{\tau}^{\alpha} s_{\mathrm{a}}^{\alpha}} \begin{cases}\frac{1}{-i \tilde{\Omega}_{\mathcal{J}}} \lim _{\zeta \rightarrow 0} \zeta\left(\hat{\Psi}_{n}\left(\zeta \Lambda_{\mathcal{J}}+i \tilde{\Omega}_{\mathcal{J}}\right)-\hat{\Psi}_{n}\left(\zeta s_{\mathrm{a}}\right)\right) & \text { for } \tilde{\Omega}_{\mathcal{J}} \neq 0 \\
\frac{1}{s_{\mathrm{a}}-\Lambda_{\mathcal{J}}} \lim _{\zeta \rightarrow 0}\left(\hat{\Psi}_{n}\left(\zeta \Lambda_{\mathcal{J}}\right)-\hat{\Psi}_{n}\left(\zeta s_{\mathrm{a}}\right)\right) & \text { for } \tilde{\Omega}_{\mathcal{J}}=0\end{cases}
\end{aligned}
$$

$=0$.

Therefore with Eq. (6.5)

$$
\lim _{\zeta \rightarrow 0} \zeta^{1+\alpha} \hat{R}_{\{1, \ldots, 2 p\} \mathcal{L}_{\mathrm{f}}}^{\mathrm{fw}}\left(\left\{\zeta \lambda_{k}+i \tilde{\omega}_{k}\right\}, \zeta s_{\mathrm{a}}\right)=0
$$

and only the term with $\mathcal{L}_{\mathrm{f}}=\{1, \ldots, 2 p\}$ remains in Eq. (10.19)

$$
\begin{aligned}
& \lim _{\zeta \rightarrow 0} \zeta^{p \alpha+1}\left|\hat{C}_{\{1, \ldots, 2 p\}}^{\text {aged }}\left(\left\{\zeta \lambda_{k}+i \tilde{\omega}_{k}\right\}, s_{\mathrm{a}}\right)\right\rangle \\
& =\lim _{\zeta \rightarrow 0} \zeta \hat{R}_{\{1, \ldots, 2 p\}\{1, \ldots, 2 p\}}^{\mathrm{fw}}\left(\left\{\zeta \lambda_{k}+i \tilde{\omega}_{k}\right\}, \zeta s_{\mathrm{a}}\right) \zeta^{p \alpha}\left|\hat{C}_{\{1, \ldots, 2 p\}}\left(\left\{\zeta \lambda_{k}+i \tilde{\omega}_{k}\right\}\right)\right\rangle \\
& =\frac{1}{s_{\mathrm{a}}-\Lambda_{\{1, \ldots, 2 p\}}} \lim _{\zeta \rightarrow 0}\left[\mathbf{I}-\hat{\Psi}_{0}\left(\zeta s_{\mathrm{a}}\right) M\right]^{-1}\left(\hat{\Psi}_{0}\left(\zeta \Lambda_{\{1, \ldots, 2 p\}}\right)-\hat{\Psi}_{0}\left(\zeta s_{\mathrm{a}}\right)\right)
\end{aligned}
$$

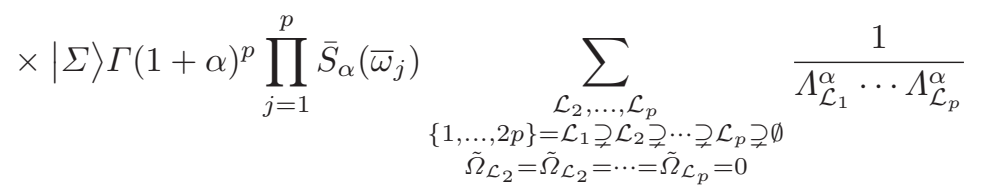

$$
\begin{aligned}
& =|\Sigma\rangle \Gamma(1+\alpha)^{p} \prod_{j=1}^{p} \bar{S}_{\alpha}\left(\bar{\omega}_{j}\right) \quad \sum_{\mathcal{L}_{2}, \ldots, \mathcal{L}_{p}} \quad \frac{s_{\mathrm{a}}^{\alpha}-\Lambda_{\mathcal{L}_{1}}^{\alpha}}{s_{\mathrm{a}}^{\alpha}\left(s_{\mathrm{a}}-\Lambda_{\mathcal{L}_{1}}\right)} \frac{1}{\Lambda_{\mathcal{L}_{1}}^{\alpha} \cdots \Lambda_{\mathcal{L}_{p}}^{\alpha}} \\
& \begin{array}{c}
\{1, \ldots, 2 p\}=\mathcal{L}_{1} \supsetneq \mathcal{L}_{2} \supsetneq \cdots \supsetneq \mathcal{L}_{p} \supsetneq \emptyset \\
\tilde{\Omega}_{\mathcal{L}_{2}}=\tilde{\Omega}_{\mathcal{L}_{2}}=\cdots=\tilde{\Omega}_{\mathcal{L}_{p}}=0
\end{array}
\end{aligned}
$$

where we used

$$
\begin{aligned}
\lim _{\zeta \rightarrow 0} & {\left[\mathbf{I}-\hat{\Psi}_{0}\left(\zeta s_{\mathrm{a}}\right) M\right]^{-1}\left(\hat{\Psi}_{0}\left(\zeta \Lambda_{\{1, \ldots, 2 p\}}\right)-\hat{\Psi}_{0}\left(\zeta s_{\mathrm{a}}\right)\right)|\Sigma\rangle } \\
& =|\Sigma\rangle \frac{1}{\bar{\tau}^{\alpha} s_{\mathrm{a}}^{\alpha}} \lim _{\zeta \rightarrow 0} \zeta^{-\alpha}\left\langle\mathrm{eq}_{\mathrm{M}}\left|\hat{\Psi}_{0}\left(\zeta \Lambda_{\{1, \ldots, 2 p\}}\right)-\hat{\Psi}_{0}\left(\zeta s_{\mathrm{a}}\right)\right| \Sigma\right\rangle \\
& =|\Sigma\rangle \frac{1}{\bar{\tau}^{\alpha} s_{\mathrm{a}}^{\alpha}} \lim _{\zeta \rightarrow 0} \zeta^{-\alpha}\left(\left\langle\mathrm{eq}_{\mathrm{M}}\left|\hat{\Psi}_{0}(0)-\hat{\Psi}_{0}\left(\zeta s_{\mathrm{a}}\right)\right| \Sigma\right\rangle-\left\langle\mathrm{eq}_{\mathrm{M}}\left|\hat{\Psi}_{0}(0)-\hat{\Psi}_{0}\left(\zeta \Lambda_{\{1, \ldots, 2 p\}}\right)\right| \Sigma\right\rangle\right) \\
& =|\Sigma\rangle \frac{1}{\bar{\tau}^{\alpha} s_{\mathrm{a}}^{\alpha}}\left(\bar{\tau}^{\alpha} s_{\mathrm{a}}^{\alpha}-\bar{\tau}^{\alpha} \Lambda_{\{1, \ldots, 2 p\}}^{\alpha}\right) .
\end{aligned}
$$

Now we go back to the Laplace transform

$$
\begin{aligned}
& \int_{0}^{\infty} \mathrm{d} T \int_{0}^{\infty} \mathrm{d} t_{\mathrm{a}} e^{-s T-s_{\mathrm{a}} t_{\mathrm{a}}} \lim _{\zeta \rightarrow 0} \zeta^{p(\alpha-1)}\left\langle S_{T / \zeta}^{\mathrm{aged}}\left(\omega_{1} ; t_{\mathrm{a}} / \zeta\right)^{q_{1}} \cdots S_{T / \zeta}^{\text {aged }}\left(\omega_{r} ; t_{\mathrm{a}} / \zeta\right)^{q_{r}}\right\rangle_{\langle\text {init }|} \\
& =\left.\int_{0}^{\infty} \mathrm{d} T \int_{0}^{\infty} \mathrm{d} t_{\mathrm{a}} e^{-s T-s_{\mathrm{a}} t_{\mathrm{a}}} \lim _{\zeta \rightarrow 0} \zeta^{p \alpha}\left\langle F_{T_{1} / \zeta}^{\text {aged }}\left(-\tilde{\omega}_{1} ; t_{\mathrm{a}} / \zeta\right) \cdots F_{T_{2 p} / \zeta}^{\mathrm{aged}}\left(-\tilde{\omega}_{2 p} ; t_{\mathrm{a}} / \zeta\right)\right\rangle_{\langle\text {init }|}\right|_{T_{1}=\cdots=T_{2 p}=T}
\end{aligned}
$$


We have calculated the Laplace transform of the right side for the different $T_{1}, \ldots, T_{2 p}$

$$
\begin{aligned}
& \mathrm{L}\left[\lim _{\zeta \rightarrow 0} \zeta^{p \alpha}\left\langle F_{T_{1} / \zeta}^{\text {aged }}\left(-\tilde{\omega}_{1} ; t_{\mathrm{a}} / \zeta\right) \cdots F_{T_{2 p} / \zeta}^{\mathrm{aged}}\left(-\tilde{\omega}_{2 p} ; t_{\mathrm{a}} / \zeta\right)\right\rangle_{\langle\text {init }|}\right]\left(\lambda_{1}, \ldots, \lambda_{2 p}, s_{\mathrm{a}}\right) \\
& =\frac{\zeta^{p \alpha}}{\lambda_{1} \cdots \lambda_{2 p}}\left\langle\text { init } \mid \hat{C}_{\{1, \ldots, 2 p\}}^{\text {aged }}\left(\left\{\zeta \lambda_{k}+i \tilde{\omega}_{k}\right\} ; \zeta s_{\mathrm{a}}\right)\right\rangle \\
& =\Gamma(1+\alpha)^{p} \prod_{j=1}^{p} \bar{S}_{\alpha}\left(\bar{\omega}_{j}\right) \quad \sum_{\mathcal{L}_{2}, \ldots, \mathcal{L}_{p}} \quad \frac{1}{\lambda_{1} \cdots \lambda_{2 p}} \frac{s_{\mathrm{a}}^{\alpha}-\Lambda_{\mathcal{L}_{1}}^{\alpha}}{s_{\mathrm{a}}^{\alpha}\left(s_{\mathrm{a}}-\Lambda_{\mathcal{L}_{1}}\right)} \frac{1}{\Lambda_{\mathcal{L}_{1}}^{\alpha} \cdots \Lambda_{\mathcal{L}_{p}}^{\alpha}} . \\
& \{1, \ldots, 2 p\}=\mathcal{L}_{1} \supsetneq \mathcal{L}_{2} \supsetneq \ldots \supsetneq \mathcal{L}_{p} \supsetneq \emptyset \\
& \tilde{\Omega}_{\mathcal{L}_{2}}=\tilde{\Omega}_{\mathcal{L}_{2}}=\cdots=\tilde{\Omega}_{\mathcal{L}_{p}}=0
\end{aligned}
$$

This case for evaluating a multidimensional Laplace transform at a point is treated in Appendix A. We can apply Eq. (A.2) here:

$$
\begin{aligned}
\int_{0}^{\infty} \mathrm{d} T \int_{0}^{\infty} \mathrm{d} t_{\mathrm{a}} e^{-s T-s_{\mathrm{a}} t_{\mathrm{a}}} \mathrm{L}^{-1}\left[\frac{1}{\lambda_{1} \cdots \lambda_{2 p}} \frac{s_{\mathrm{a}}^{\alpha}-\Lambda_{\mathcal{L}_{1}}^{\alpha}}{s_{\mathrm{a}}^{\alpha}\left(s_{\mathrm{a}}-\Lambda_{\mathcal{L}_{1}}\right)} \frac{1}{\Lambda_{\mathcal{L}_{1}}^{\alpha} \cdots \Lambda_{\mathcal{L}_{p}}^{\alpha}}\right]\left(T_{1}\right. & \left.=\cdots=T_{2 p}=T, t_{\mathrm{a}}\right) \\
& =\frac{1}{s^{p+1}} \frac{s_{\mathrm{a}}^{\alpha}-s^{\alpha}}{s_{\mathrm{a}}^{\alpha}\left(s_{\mathrm{a}}-s\right)} .
\end{aligned}
$$

We use the combinatorial result Eq. (9.34) to get in Eq. (10.24)

$$
\begin{aligned}
& \int_{0}^{\infty} \mathrm{d} T \int_{0}^{\infty} \mathrm{d} t_{\mathrm{a}} e^{-s T-s_{\mathrm{a}} t_{\mathrm{a}}} \lim _{\zeta \rightarrow 0} \zeta^{p(\alpha-1)}\left\langle S_{T / \zeta}^{\text {aged }}\left(\omega_{1} ; t_{\mathrm{a}} / \zeta\right)^{q_{1}} \cdots S_{T / \zeta}^{\text {aged }}\left(\omega_{r} ; t_{\mathrm{a}} / \zeta\right)^{q_{r}}\right\rangle_{\langle\text {init }|} \\
& =\Gamma(1+\alpha)^{p} \prod_{j=1}^{p} \bar{S}_{\alpha}\left(\bar{\omega}_{j}\right) \sum_{\substack{\mathcal{L}_{2}, \ldots, \mathcal{L}_{p} \\
\{1, \ldots, 2 p\}=\mathcal{L}_{1} \supsetneq \mathcal{L}_{2} \supsetneq \cdots \supsetneq \mathcal{L}_{p+1}=\emptyset \\
\tilde{\Omega}_{\mathcal{L}_{2}}=\tilde{\Omega}_{\mathcal{L}_{2}}=\cdots=\tilde{\Omega}_{\mathcal{L}_{p}=0}}} \frac{1}{s^{p \alpha+1}} \frac{s_{\mathrm{a}}^{\alpha}-s^{\alpha}}{s_{\mathrm{a}}^{\alpha}\left(s_{\mathrm{a}}-s\right)} \\
& =\frac{\Gamma(1+\alpha)^{p} p !\left(s_{\mathrm{a}}^{\alpha}-s^{\alpha}\right)}{s^{p \alpha+1} s_{\mathrm{a}}^{\alpha}\left(s_{\mathrm{a}}-s\right)} \prod_{j=1}^{r} q_{j} ! \bar{S}_{\alpha}\left(\omega_{j}\right)^{q_{j}} .
\end{aligned}
$$

The inverse Laplace transform of $\frac{p !\left(s_{\mathrm{a}}^{\alpha}-s^{\alpha}\right)}{s^{p \alpha+1} s_{\mathrm{a}}^{\alpha}\left(s_{\mathrm{a}}-s\right)}$ is given in [46] (Eq. (8) there)

$$
\mathrm{L}^{-1}\left[\frac{p !\left(s_{\mathrm{a}}^{\alpha}-s^{\alpha}\right)}{s^{p \alpha+1} s_{\mathrm{a}}^{\alpha}\left(s_{\mathrm{a}}-s\right)}\right]=T^{p \alpha} \frac{p !}{\Gamma(\alpha) \Gamma(1+p \alpha-\alpha)}\left(1+\frac{t_{\mathrm{a}}}{T}\right)^{p \alpha} \mathrm{B}\left(\left(1+t_{\mathrm{a}} / T\right)^{-1} ; 1+p \alpha-\alpha, \alpha\right)
$$

with the incomplete beta function $\mathrm{B}(z ; a, b)$. We define therefore a random variable $Z_{\alpha, \xi}$ by its moments

$$
\left\langle Z_{\alpha, \xi}^{p}\right\rangle=\frac{\Gamma(1+\alpha)^{p} p !}{\Gamma(\alpha) \Gamma(1+p \alpha-\alpha)}(1+\xi)^{p \alpha} \mathrm{B}\left(\frac{1}{1+\xi} ; 1+p \alpha-\alpha, \alpha\right) .
$$

Its properties are discussed in [1][46]. Then we have in distribution

$$
\lim _{\substack{T, t_{\mathrm{a}} \rightarrow \infty \\ t_{\mathrm{a}} / T=\text { const }}} T^{1-\alpha}\left(S_{T}^{\text {aged }}\left(\omega_{1} ; t_{\mathrm{a}}\right), \ldots, S_{T}^{\text {aged }}\left(\omega_{r} ; t_{\mathrm{a}}\right)\right)=Z_{\alpha, t_{\mathrm{a}} / T}\left(E_{1} \bar{S}_{\alpha}\left(\omega_{1}\right), \ldots, E_{r} \bar{S}_{\alpha}\left(\omega_{r}\right)\right) .
$$

As discussed in [40] it is not surprising that the pre-factor has the same statistics as the number of epochs.

One should remarks however, that the expected value for the power spectrum is

$$
\begin{aligned}
\lim _{\substack{T, t_{\mathrm{a}} \rightarrow \infty \\
t_{\mathrm{a}} / T=\text { const }}} T^{1-\alpha}\left\langle S_{T}^{\text {aged }}\left(\omega ; t_{\mathrm{a}}\right)\right\rangle & =\left\langle Y_{\alpha, t_{\mathrm{a}} / T}\right\rangle \bar{S}_{\alpha}(\omega) \\
& =\left(\left(1+t_{\mathrm{a}} / T\right)^{\alpha}-\left(t_{\mathrm{a}} / T\right)^{\alpha}\right) \bar{S}_{\alpha}(\omega) .
\end{aligned}
$$




\section{Summary}

In this article we have introduced a general renewal process with internal states. The internal states determine the observed signal and the waiting times while the dynamics on the internal states is Markovian. This general setting allows to treat several models previously used in a unified framework. We have used here the single state model, the switching model and the burst model as examples and we have restricted ourself to a finite internal state space. A CTRW with power law waiting times and a finite number of states [14] would be an example for an $N$-state model with $N>2$. In [51] particles jumping between traps with power law distributed sojourn times have been detected experimentally, where our theory should apply, see also [50]. In dynamical systems theory, maps of Pomeau-Manneville type generate power law distributed sojourn types due to dynamical intermittency. One might consider such systems in a setting where the number of marginally unstable fixed points, which can be designed by a piecewise definition of the map, as the number of internal states and thereby easily go beyond $N=2$. But there seems to be no conceptual barrier in expanding the dynamics $M$ on the internal state space to an ergodic Markov chain on infinitely many states as long as one is able to perform a perturbation expansion of the stationary state (Eqs. (7.11), (8.10)). So it would be intersting to study, e.g., a fractional time Fokker Planck equation using a subordination scheme [19].

We have derived the technical tools for working with this type of process. Starting from a generalized renewal ansatz, we have introduced a recursion formula for the general multi-time joint probability distributions (Eq. (3.18)) and the correlation functions (Eq. (5.9)). Additionally, we have considered aged processes. These are processes where the observation of the process starts at a later time than the process. An important quantity in this respect is the generalization of the forward recurrence time for this type of processes (Eq. (4.10)). We have also extended our tools to treat aged processes: the joint probability distributions are described by Eq. (4.14) and the correlation functions by Eq. (6.4).

A special case are the stationary processes which is the observation of processes already running for an infinite amount of time. This stationary description is valid as soon as the mean waiting time is finite. For these processes, we have calculated the power spectrum by using the Wiener-Khinchin theorem (Eq. (7.32)) from the stationary correlation function. For the case that the second moment is infinite, we find $1 / f^{1-\beta}$ noise.

We have used our tools to derive the statistical properties of observing the power spectrum in the general case and thereby giving a complete derivation of the results motivated in [40]. We have focused here in the periodogram estimator as the behavior of many other spectral estimators can be derived from this one. When the mean waiting time is infinite, i.e., the waiting time distribution has a long time tail $\sim 1 / t^{1+\alpha}$ with $0<\alpha<1$, the spectral observable decays with measurement time as $T^{\alpha-1}$. We also find a $1 / f^{2-\alpha}$ noise in case the long time variance $\sigma_{\mathrm{lt}}^{2}>0$. This is true for many processes encountered in practice; we get a different behavior when the long time waiting times are completely dominated by a single value of the signal. An example of an exception is the burst process where we have a different $1 / f^{\gamma}$ noise (Eqs. (8.36), (8.37)).

The fluctuations of the periodogram estimator of the spectrum for several frequencies is described by Eq. (9.37). Each frequency has an independent fluctuation in the form of an exponential distribution. However there is a common random pre-factor $Y_{\alpha}$ of Mittag-Leffler type. Many smoothing techniques for estimating the spectrum (e.g., binning) smooth the exponential distributions but they cannot account for the Mittag-Leffler pre-factor, i.e., the $1 / f$ form of the spectrum is established but the exact values will remain random. For a more thorough discussion of the implications for the measurement process and numerical simulations we refer to [40].

In the last section, we have looked at the fluctuations for aged processes. If the aged time is small compared to the observation time, we do not see a difference to a process without aging in the limit of long observation times. However, if the aged time is of the order of the observation time, the Mittag-Leffler pre-factor $Y_{\alpha}$ is replaced by a more general random variable $Z_{\alpha, t_{\mathrm{a}} / T}$ (Eq. (10.30)) which appears also as the number of renewals of an aged process [1][46]. 


\section{A. Inversion of a Laplace transform}

In this appendix we are deriving a method of calculating a specific type of multidimensional inverse Laplace transforms when one evaluates them at the same point in time space. To formulate the result we take a $n$-dimensional function $f\left(t_{1}, \ldots, t_{n}\right)$ and its Laplace transform

$$
\begin{aligned}
\hat{f}\left(\lambda_{1}, \ldots, \lambda_{n}\right) & =\mathrm{L}\left[f\left(T_{1}, \ldots, T_{n}\right)\right]\left(\lambda_{1}, \ldots, \lambda_{n}\right) \\
& =\int_{0}^{\infty} \mathrm{d} t_{1} \cdots \int_{0}^{\infty} \mathrm{d} t_{n} \exp \left(-\left(\lambda_{1} T_{1}+\cdots+\lambda_{n} T_{n}\right)\right) f\left(T_{1}, \ldots, T_{n}\right) .
\end{aligned}
$$

For any nested sequence of $n$ subsets $\{1, \ldots, n\}=\mathcal{L}_{1} \subsetneq \mathcal{L}_{2} \subsetneq \cdots \subsetneq \mathcal{L}_{n} \subsetneq \emptyset$ (i.e., each $\mathcal{L}_{i} \backslash \mathcal{L}_{i+1}$ contains exactly one element). In this case we have a law similar to the convolution property

$$
\int_{0}^{\infty} \mathrm{d} t e^{-s t} \mathrm{~L}^{-1}\left[\frac{1}{\lambda_{1} \cdots \lambda_{n}} \hat{f}\left(\Lambda_{\mathcal{L}_{n}}, \Lambda_{\mathcal{L}_{n-1}}, \ldots, \Lambda_{\mathcal{L}_{2}}, \Lambda_{\mathcal{L}_{1}}\right)\right]\left(T_{1}=\ldots=T_{n}=t\right)=\frac{1}{s} \hat{f}(s, \ldots, s) .
$$

As it is symmetric with respect to the indices, it is enough to consider the case $\mathcal{L}_{1}=\{1, \ldots, n\}, \mathcal{L}_{2}=$ $\{1, \ldots, n-1\}, \ldots, \mathcal{L}_{n-1}=\{1,2\}, \mathcal{L}_{n}=\{1\}:$

$$
\int_{0}^{\infty} \mathrm{d} t e^{-s t} \mathrm{~L}^{-1}\left[\frac{1}{\lambda_{1} \cdots \lambda_{n}} \hat{f}\left(\Lambda_{\{1\}}, \Lambda_{\{1,2\}}, \ldots, \Lambda_{\{1, \ldots, n\}}\right)\right]\left(T_{1}=\ldots=T_{n}=t\right)=\frac{1}{s} \hat{f}(s, \ldots, s) .
$$

In first step, we determine the inverse Laplace transform of $\hat{f}\left(\Lambda_{\{1\}}, \Lambda_{\{1,2\}}, \ldots, \Lambda_{\{1, \ldots, n\}}\right)$. We start with

$$
\begin{aligned}
\mathrm{L} & {\left[f\left(T_{1}-T_{2}, T_{2}-T_{3}, \ldots, T_{n-1}-T_{n}, T_{n}\right) \theta\left(T_{1}-T_{2}\right) \cdots \theta\left(T_{n-1}-T_{n}\right)\right]\left(\lambda_{1}, \ldots, \lambda_{n}\right) } \\
& =\int_{0}^{\infty} \mathrm{d} T_{n} \int_{T_{n}}^{\infty} \mathrm{d} T_{n-1} \cdots \int_{T_{2}}^{\infty} \mathrm{d} T_{1} \exp \left(-\left(\lambda_{1} T_{1}+\cdots+\lambda_{n} T_{n}\right)\right) f\left(T_{1}-T_{2}, T_{2}-T_{3}, \ldots, T_{n-1}-T_{n}, T_{n}\right)
\end{aligned}
$$

where $\theta(t)$ is the Heaviside step function. Doing the substitutions $t_{1}=T_{1}-T_{2}, t_{2}=T_{3}-T_{2}, \ldots$, $t_{n-1}=T_{n-1}-T_{n}, t_{n}=T_{n}$ gives

$$
\begin{aligned}
\mathrm{L} & {\left[f\left(T_{1}-T_{2}, T_{2}-T_{3}, \ldots, T_{n-1}-T_{n}, T_{n}\right) \theta\left(T_{1}-T_{2}\right) \cdots \theta\left(T_{n-1}-T_{n}\right)\right]\left(\lambda_{1}, \ldots, \lambda_{n}\right) } \\
& =\int_{0}^{\infty} \mathrm{d} t_{n} \int_{0}^{\infty} \mathrm{d} t_{n-1} \cdots \int_{0}^{\infty} \mathrm{d} t_{1} \exp \left(-\left(\Lambda_{\{1\}} t_{1}+\Lambda_{\{1,2\}} t_{2}+\cdots+\Lambda_{\{1, \ldots, n\}} t_{n}\right)\right) f\left(t_{1}, t_{2}, \ldots, t_{n-1}, t_{n}\right) \\
& =\hat{f}\left(\Lambda_{\{1\}}, \Lambda_{\{1,2\}}, \ldots, \Lambda_{\{1, \ldots, n\}}\right) .
\end{aligned}
$$

Therefore the inverse Laplace transform of $\hat{f}\left(\Lambda_{\{1\}}, \Lambda_{\{1,2\}}, \ldots, \Lambda_{\{1, \ldots, n\}}\right)$ is $f\left(T_{1}-T_{2}, T_{2}-T_{3}, \ldots, T_{n-1}-\right.$ $\left.T_{n}, T_{n}\right) \theta\left(T_{1}-T_{2}\right) \cdots \theta\left(T_{n-1}-T_{n}\right)$.

As a next step, we combine this with the integration property of the Laplace transform

$$
\begin{aligned}
& \int_{0}^{\infty} \mathrm{d} t e^{-s t} \mathrm{~L}^{-1}\left[\frac{1}{\lambda_{1} \cdots \lambda_{n}} \hat{f}\left(\Lambda_{\{1\}}, \Lambda_{\{1,2\}}, \ldots, \Lambda_{\{1, \ldots, n\}}\right)\right]\left(T_{1}=\cdots=T_{n}=t\right) \\
& =\int_{0}^{\infty} \mathrm{d} t e^{-s t} \int_{0}^{t} \mathrm{~d} T_{1} \int_{0}^{t} \mathrm{~d} T_{2} \ldots \int_{0}^{t} \mathrm{~d} T_{n} f\left(T_{1}-T_{2}, T_{2}-T_{3}, \ldots, T_{n-1}-T_{n}, T_{n}\right) \theta\left(T_{1}-T_{2}\right) \cdots \theta\left(T_{n-1}-T_{n}\right) \\
& =\int_{0}^{\infty} \mathrm{d} t e^{-s t} \int_{0}^{t} \mathrm{~d} T_{1} \int_{0}^{T_{1}} \mathrm{~d} T_{2} \cdots \int_{0}^{T_{n-1}} \mathrm{~d} T_{n} f\left(T_{1}-T_{2}, T_{2}-T_{3}, \ldots, T_{n-1}-T_{n}, T_{n}\right) \\
& =\frac{1}{s} \int_{0}^{\infty} \mathrm{d} T_{1} \int_{0}^{T_{1}} \mathrm{~d} T_{2} \cdots \int_{0}^{T_{n-1}} \mathrm{~d} T_{n} e^{-s T_{1}} f\left(T_{1}-T_{2}, T_{2}-T_{3}, \ldots, T_{n-1}-T_{n}, T_{n}\right) \\
& =\frac{1}{s} \int_{0}^{\infty} \mathrm{d} T_{n} \int_{T_{n}}^{\infty} \mathrm{d} T_{n-1} \cdots \int_{T_{2}}^{\infty} \mathrm{d} T_{1} e^{-s T_{1}} f\left(T_{1}-T_{2}, T_{2}-T_{3}, \ldots, T_{n-1}-T_{n}, T_{n}\right) .
\end{aligned}
$$


Doing again the substitution $t_{1}=T_{1}-T_{2}, t_{2}=T_{3}-T_{2}, \ldots, t_{n-1}=T_{n-1}-T_{n}, t_{n}=T_{n}$ gives

$$
\begin{aligned}
\int_{0}^{\infty} \mathrm{d} t e^{-s t} & \mathrm{~L}^{-1}\left[\frac{1}{\lambda_{1} \cdots \lambda_{n}} \hat{f}\left(\Lambda_{\{1\}}, \Lambda_{\{1,2\}}, \ldots, \Lambda_{\{1, \ldots, n\}}\right)\right]\left(T_{1}=\cdots=T_{n}=t\right) \\
& =\frac{1}{s} \int_{0}^{\infty} \mathrm{d} t_{n} \int_{0}^{\infty} \mathrm{d} t_{n-1} \cdots \int_{0}^{\infty} \mathrm{d} t_{1} e^{-s\left(t_{1}+\cdots+t_{n}\right)} f\left(t_{1}, \ldots, t_{n}\right) \\
& =\frac{1}{s} \hat{f}(s, \ldots, s) .
\end{aligned}
$$

Which is Eq. (A.2) as we wanted to show.

We can use this result to evaluate

$$
\mathrm{L}^{-1}\left[\frac{1}{\lambda_{1} \cdots \lambda_{2 p}} \frac{1}{\Lambda_{\{1, \ldots, 2 p\}}^{\alpha} \Lambda_{\{1, \ldots, 2 p-2\}}^{\alpha} \cdots \Lambda_{\{1,2\}}^{\alpha}}\right]\left(T_{1}=\cdots=T_{2 p}=t\right)
$$

Applying Eq. (A.2) gives

$$
\int_{0}^{\infty} \mathrm{d} t e^{-s t} \mathrm{~L}^{-1}\left[\frac{1}{\lambda_{1} \cdots \lambda_{2 p}} \frac{1}{\Lambda_{\{1, \ldots, 2 p\}}^{\alpha} \Lambda_{\{1, \ldots, 2 p-2\}}^{\alpha} \cdots \Lambda_{\{1,2\}}^{\alpha}}\right]\left(T_{1}=\cdots=T_{2 p}=t\right)=\frac{1}{s^{1+p \alpha}}
$$

which can be solved by a simple one-dimension Laplace inversion

$$
\mathrm{L}^{-1}\left[\frac{1}{\lambda_{1} \cdots \lambda_{2 p}} \frac{1}{\Lambda_{\{1, \ldots, 2 p\}}^{\alpha} \Lambda_{\{1, \ldots, 2 p-2\}}^{\alpha} \cdots \Lambda_{\{1,2\}}^{\alpha}}\right]\left(T_{1}=\cdots=T_{2 p}=t\right)=\frac{t^{p \alpha}}{\Gamma(1+p \alpha)}
$$

\section{B. Low-frequency behavior in the case of a finite mean time}

As in Sec. 7 above we will determine $\left[\mathbf{I}-\hat{\Psi}_{0}(i \omega) M\right]^{-1}$ by consider the perturbation Eq. (7.33) with $0<\beta<1$ :

$$
\mathbf{I}-\hat{\Psi}_{0}(i \omega) M=\mathbf{I}-M+i \omega K_{0} M-(i \omega)^{1+\beta} L_{0} M+\mathrm{o}\left(\omega^{1+\beta}\right)
$$

We can still work with first order perturbation theory, as the first term of second order will be of the order $\mathrm{O}(1)$. Therefore,

$$
\begin{aligned}
{\left[\mathbf{I}-\hat{\Psi}_{0}(i \omega) M\right]^{-1} } & =\frac{|\Sigma\rangle\left\langle\mathrm{eq}_{\mathrm{M}}\right|+\mathrm{O}(\omega)}{i \omega\left\langle\mathrm{eq}_{\mathrm{M}}\left|K_{0}\right| \Sigma\right\rangle-(i \omega)^{1+\beta}\left\langle\mathrm{eq}_{\mathrm{M}}\left|L_{0}\right| \Sigma\right\rangle+\mathrm{o}\left(\omega^{1+\beta}\right)} \\
& =\frac{|\Sigma\rangle\left\langle\mathrm{eq}_{\mathrm{M}}\right|}{i \omega\left\langle\mathrm{eq}_{\mathrm{M}}\left|K_{0}\right| \Sigma\right\rangle}\left(1+(i \omega)^{\beta} \frac{\left\langle\mathrm{eq}_{\mathrm{M}}\left|L_{0}\right| \Sigma\right\rangle}{\left\langle\mathrm{eq}_{\mathrm{M}}\left|K_{0}\right| \Sigma\right\rangle}\right)+\mathrm{o}\left(\omega^{\beta-1}\right) .
\end{aligned}
$$


Plugging this expansion in Eq. (7.32) gives $(\omega>0)$

$$
\begin{aligned}
& \bar{S}(\omega)=-\frac{1}{\omega^{2}} \frac{1}{\left\langle\mathrm{eq}_{\mathrm{M}}\left|K_{0}\right| \Sigma\right\rangle} \\
& \left(\left\langle\mathrm{eq}_{\mathrm{M}}\left|\left(i \omega K_{1}-(i \omega)^{1+\beta} L_{1}\right) M \frac{|\Sigma\rangle\left\langle\mathrm{eq}_{\mathrm{M}}\right|}{i \omega\left\langle\mathrm{eq}_{\mathrm{M}}\left|K_{0}\right| \Sigma\right\rangle}\left(1+(i \omega)^{\beta} \frac{\left\langle\mathrm{eq}_{\mathrm{M}}\left|L_{0}\right| \Sigma\right\rangle}{\left\langle\mathrm{eq}_{\mathrm{M}}\left|K_{0}\right| \Sigma\right\rangle}\right)\left(i \omega K_{1}-(i \omega)^{1+\beta} L_{1}\right)\right| \Sigma\right\rangle\right. \\
& +\left\langle\mathrm{eq}_{\mathrm{M}}\left|\left(-i \omega K_{1}-(-i \omega)^{1+\beta} L_{1}\right) M \frac{|\Sigma\rangle\left\langle\mathrm{eq}_{\mathrm{M}}\right|}{-i \omega\left\langle\mathrm{eq}_{\mathrm{M}}\left|K_{0}\right| \Sigma\right\rangle}\left(1+(-i \omega)^{\beta} \frac{\left\langle\mathrm{eq}_{\mathrm{M}}\left|L_{0}\right| \Sigma\right\rangle}{\left\langle\mathrm{eq}_{\mathrm{M}}\left|K_{0}\right| \Sigma\right\rangle}\right)\left(-i \omega K_{1}-(-i \omega)^{1+\beta} L_{1}\right)\right| \Sigma\right\rangle \\
& \left.+\left\langle\mathrm{eq}_{\mathrm{M}}\left|(i \omega)^{1+\beta} L_{2}+(-i \omega)^{1+\beta} L_{2}\right| \Sigma\right\rangle\right)+\mathrm{o}\left(\omega^{\beta-1}\right) \\
& =-\frac{i}{\omega}\left(\frac{\left\langle\mathrm{eq}_{\mathrm{M}}\left|K_{1}-(i \omega)^{\beta} L_{1}\right| \Sigma\right\rangle}{\left\langle\mathrm{eq}_{\mathrm{M}}\left|K_{0}\right| \Sigma\right\rangle}\right)^{2}\left(1+(i \omega)^{\beta} \frac{\left\langle\mathrm{eq}_{\mathrm{M}}\left|L_{0}\right| \Sigma\right\rangle}{\left\langle\mathrm{eq}_{\mathrm{M}}\left|K_{0}\right| \Sigma\right\rangle}\right) \\
& +\frac{i}{\omega}\left(\frac{\left\langle\mathrm{eq}_{\mathrm{M}}\left|K_{1}-(-i \omega)^{\beta} L_{1}\right| \Sigma\right\rangle}{\left\langle\mathrm{eq}_{\mathrm{M}}\left|K_{0}\right| \Sigma\right\rangle}\right)^{2}\left(1+(-i \omega)^{\beta} \frac{\left\langle\mathrm{eq}_{\mathrm{M}}\left|L_{0}\right| \Sigma\right\rangle}{\left\langle\mathrm{eq}_{\mathrm{M}}\left|K_{0}\right| \Sigma\right\rangle}\right) \\
& -\frac{(i \omega)^{1+\beta}+(-i \omega)^{1+\beta}}{\omega^{2}} \frac{\left\langle\mathrm{eq}_{\mathrm{M}}\left|L_{2}\right| \Sigma\right\rangle}{\left\langle\mathrm{eq}_{\mathrm{M}}\left|K_{0}\right| \Sigma\right\rangle}+\mathrm{o}\left(\omega^{\beta-1}\right) \\
& =\frac{(i \omega)^{\beta}-(-i \omega)^{\beta}}{i \omega}\left(\frac{\left\langle\mathrm{eq}_{\mathrm{M}}\left|K_{1}\right| \Sigma\right\rangle^{2}\left\langle\mathrm{eq}_{\mathrm{M}}\left|L_{0}\right| \Sigma\right\rangle}{\left\langle\mathrm{eq}\left|K_{0}\right| \Sigma\right\rangle^{3}}-2 \frac{\left\langle\mathrm{eq}_{\mathrm{M}}\left|K_{1}\right| \Sigma\right\rangle\left\langle\mathrm{eq}_{\mathrm{M}}\left|L_{1}\right| \Sigma\right\rangle}{\left\langle\mathrm{eq}\left|K_{0}\right| \Sigma\right\rangle^{2}}+\frac{\left\langle\mathrm{eq}_{\mathrm{M}}\left|L_{2}\right| \Sigma\right\rangle}{\left\langle\mathrm{eq}_{\mathrm{M}}\left|K_{0}\right| \Sigma\right\rangle}\right)+\mathrm{o}\left(\omega^{\beta-1}\right) .
\end{aligned}
$$

The $\omega$ dependence can be transformed via (the branch cut is at the negative real axis)

$$
\begin{aligned}
\frac{(i \omega)^{\beta}-(-i \omega)^{\beta}}{i \omega} & =\frac{1}{\omega^{1-\beta}} \frac{i^{\beta}-(-i)^{\beta}}{i} \\
& =\frac{1}{\omega^{1-\beta}} \frac{\exp \left(i \beta \frac{\pi}{2}\right)-\exp \left(-i \beta \frac{\pi}{2}\right)}{i} \\
& =\frac{2 \sin \left(\beta \frac{\pi}{2}\right)}{\omega^{1-\beta}} .
\end{aligned}
$$

And we have

$$
\begin{aligned}
\bar{S}(\omega) & =\left(\frac{\left\langle\mathrm{eq}_{\mathrm{M}}\left|K_{1}\right| \Sigma\right\rangle^{2}\left\langle\mathrm{eq}_{\mathrm{M}}\left|L_{0}\right| \Sigma\right\rangle}{\left\langle\mathrm{eq}_{\mathrm{M}}\left|K_{0}\right| \Sigma\right\rangle^{3}}-2 \frac{\left\langle\mathrm{eq}_{\mathrm{M}}\left|K_{1}\right| \Sigma\right\rangle\left\langle\mathrm{eq}_{\mathrm{M}}\left|L_{1}\right| \Sigma\right\rangle}{\left\langle\mathrm{eq}_{\mathrm{M}}\left|K_{0}\right| \Sigma\right\rangle^{2}}+\frac{\left\langle\mathrm{eq}_{\mathrm{M}}\left|L_{2}\right| \Sigma\right\rangle}{\left\langle\mathrm{eq}_{\mathrm{M}}\left|K_{0}\right| \Sigma\right\rangle}\right) \frac{2 \sin \left(\beta \frac{\pi}{2}\right)}{\omega^{1-\beta}}+\mathrm{o}\left(\omega^{\beta-1}\right) \\
& =\left[\frac{\left\langle\mathrm{eq}_{\mathrm{M}}\left|L_{2}\right| \Sigma\right\rangle}{\left\langle\mathrm{eq}_{\mathrm{M}}\left|L_{0}\right| \Sigma\right\rangle}-2 \frac{\left\langle\mathrm{eq}_{\mathrm{M}}\left|L_{1}\right| \Sigma\right\rangle\left\langle\mathrm{eq}_{\mathrm{M}}\left|K_{1}\right| \Sigma\right\rangle}{\left\langle\mathrm{eq}_{\mathrm{M}}\left|L_{0}\right| \Sigma\right\rangle\left\langle\mathrm{eq}_{\mathrm{M}}\left|K_{0}\right| \Sigma\right\rangle}+\frac{\left\langle\mathrm{eq}_{\mathrm{M}}\left|K_{1}\right| \Sigma\right\rangle^{2}}{\left\langle\mathrm{eq}_{\mathrm{M}}\left|K_{0}\right| \Sigma\right\rangle^{2}}\right] \frac{\left\langle\mathrm{eq}_{\mathrm{M}}\left|L_{0}\right| \Sigma\right\rangle}{\left\langle\mathrm{eq}_{\mathrm{M}}\left|K_{0}\right| \Sigma\right\rangle} \frac{2 \sin \left(\beta \frac{\pi}{2}\right)}{\omega^{1-\beta}}+\mathrm{o}\left(\omega^{\beta-1}\right)
\end{aligned}
$$

\section{Interpretation of the expansion terms}

We already claimed in Section 2 that the asymptotic moments

$$
\mu_{n}^{\text {asym }}=\lim _{t \rightarrow \infty}\left\langle X^{n}(t)\right\rangle_{\langle\text {init }|}
$$

can be expressed via the $K_{j}$ which are defined by the expansion

$$
\hat{\Psi}_{n}(\lambda)=\hat{\Psi}_{n}(0)-\lambda^{\alpha} K_{n}+\mathrm{o}\left(\lambda^{\alpha}\right)
$$


with $0<\alpha \leq 1$. We use Eq. (3.19) to get the Laplace transform

$$
\begin{aligned}
\int_{0}^{\infty} \mathrm{d} t \exp (-\lambda t)\left\langle X^{n}(t)\right\rangle_{\langle\text {init }|} & =\sum_{m=1}^{N} \int_{-\infty}^{\infty} \mathrm{d} x x^{n}\left\langle\operatorname{init} \mid \hat{P}_{1}(x, m ; \lambda)\right\rangle \\
& =\frac{1}{\lambda}\left\langle\operatorname{init}\left|\left[\mathbf{I}-\hat{\Psi}_{0}(\lambda) M\right]^{-1}\left(\hat{\Psi}_{n}(0)-\hat{\Psi}_{n}(\lambda)\right)\right| \Sigma\right\rangle .
\end{aligned}
$$

In Eq. (8.10) we have already determined that for small $\lambda$

$$
\left[\mathbf{I}-\hat{\Psi}_{0}(\lambda) M\right]^{-1}=\frac{|\Sigma\rangle\left\langle\mathrm{eq}_{\mathrm{M}}\right|}{\left\langle\mathrm{eq}_{\mathrm{M}}\left|K_{0}\right| \Sigma\right\rangle \lambda^{\alpha}}+\mathrm{o}\left(\lambda^{-\alpha}\right)
$$

such that with the final value theorem for the Laplace transform

$$
\begin{aligned}
\mu_{n}^{\text {asym }} & =\lim _{\lambda \rightarrow 0} \lambda \int_{0}^{\infty} \mathrm{d} t \exp (-\lambda t)\left\langle X^{n}(t)\right\rangle_{\langle\text {init }|} \\
& =\lim _{\lambda \rightarrow 0}\left\langle\text { init }\left|\left[\mathbf{I}-\hat{\Psi}_{0}(\lambda) M\right]^{-1}\left(\hat{\Psi}_{n}(0)-\hat{\Psi}_{n}(\lambda)\right)\right| \Sigma\right\rangle \\
& =\frac{\left\langle\mathrm{eq}_{\mathrm{M}}\left|K_{n}\right| \Sigma\right\rangle}{\left\langle\operatorname{eq}\left|K_{0}\right| \Sigma\right\rangle} .
\end{aligned}
$$

This result is valid both for the ergodic case $(\alpha=1)$ and the non-ergodic case $(\alpha<1)$.

In the non-ergodic case, these moments can be directly expressed by the properties of the process. They correspond to the moments of the long-Time distribution $\bar{\phi}^{\mathrm{lt}}(x)$ defined in Eq. (2.15). It describes the long-time behavior of $\bar{\phi}(x, t)=\left\langle\mathrm{eq}_{\mathrm{M}}|\Phi(x, t)| \Sigma\right\rangle$ (see Eq. (2.7))

$$
\bar{\phi}^{\mathrm{lt}}(x)=\lim _{T \rightarrow \infty} \frac{\int_{T}^{\infty} \mathrm{d} t \bar{\phi}(x, t)}{\int_{T}^{\infty} \mathrm{d} t \int_{-\infty}^{\infty} \mathrm{d} x \bar{\phi}(x, t)}
$$

with moments

$$
\begin{aligned}
\bar{\psi}_{n}^{\mathrm{lt}} & =\int_{-\infty}^{\infty} \mathrm{d} x x^{n} \bar{\phi}^{\mathrm{lt}}(x) \\
& =\lim _{T \rightarrow \infty} \frac{\int_{T}^{\infty} \mathrm{d} t \int_{-\infty}^{\infty} \mathrm{d} x x^{n} \bar{\phi}(x, t)}{\int_{T}^{\infty} \mathrm{d} t \int_{-\infty}^{\infty} \mathrm{d} x \bar{\phi}(x, t)} .
\end{aligned}
$$

Similar to [20] (Eqs. (1.6), (1.8)) we have the correspondence of the large time behavior (for $0<\alpha<1$ )

$$
\int_{T}^{\infty} \mathrm{d} t \int_{-\infty}^{\infty} \mathrm{d} x x^{n} \bar{\phi}(x, t) \simeq \frac{T^{-\alpha}}{\Gamma(1-\alpha)}\left\langle\mathrm{eq}_{\mathrm{M}}\left|K_{n}\right| \Sigma\right\rangle
$$

to the small $\lambda$ behavior

$$
\left\langle\mathrm{eq}_{\mathrm{M}}\left|\hat{\Psi}_{n}(\lambda)\right| \Sigma\right\rangle \simeq\left\langle\mathrm{eq}_{\mathrm{M}}\left|\hat{\Psi}_{n}(0)\right| \Sigma\right\rangle-\left\langle\mathrm{eq}_{\mathrm{M}}\left|K_{n}\right| \Sigma\right\rangle \lambda^{\alpha} .
$$

The long time moments are then

$$
\begin{aligned}
\bar{\psi}_{n}^{\mathrm{lt}} & =\lim _{T \rightarrow \infty} \frac{T^{-\alpha}\left\langle\mathrm{eq}_{\mathrm{M}}\left|K_{n}\right| \Sigma\right\rangle / \Gamma(1-\alpha)}{T^{-\alpha}\left\langle\mathrm{eq}_{\mathrm{M}}\left|K_{0}\right| \Sigma\right\rangle / \Gamma(1-\alpha)} \\
& =\frac{\left\langle\mathrm{eq}_{\mathrm{M}}\left|K_{n}\right| \Sigma\right\rangle}{\left\langle\mathrm{eq}_{\mathrm{M}}\left|K_{0}\right| \Sigma\right\rangle}
\end{aligned}
$$


Especially, in the $0<\alpha<1$ case, the moments of the long time distribution and the asymptotic moments of the process $X(t)$ coincide

$$
\mu_{n}^{\mathrm{asym}}=\bar{\psi}_{n}^{\mathrm{lt}}
$$

The variance $\sigma_{\mathrm{lt}}^{2}$ of $\bar{\phi}^{\mathrm{lt}}(x)$ is given by

$$
\begin{aligned}
\sigma_{\mathrm{lt}}^{2} & =\bar{\psi}_{2}^{\mathrm{lt}}-\left(\bar{\psi}_{1}^{\mathrm{lt}}\right)^{2} \\
& =\frac{\left\langle\mathrm{eq}_{\mathrm{M}}\left|K_{2}\right| \Sigma\right\rangle}{\left\langle\mathrm{eq}_{\mathrm{M}}\left|K_{0}\right| \Sigma\right\rangle}-\frac{\left\langle\mathrm{eq}_{\mathrm{M}}\left|K_{1}\right| \Sigma\right\rangle^{2}}{\left\langle\mathrm{eq}_{\mathrm{M}}\left|K_{0}\right| \Sigma\right\rangle^{2}} .
\end{aligned}
$$

This last expression scales the small frequency behavior of the spectrum (Eq. (8.22)).

In the ergodic case $(\alpha=1)$ the values of $\left\langle\mathrm{eq}_{\mathrm{M}}\left|K_{n}\right| \Sigma\right\rangle$ can be expressed with help of $\bar{\phi}(x, t)$. For this we note

$$
\begin{aligned}
\left\langle\mathrm{eq}_{\mathrm{M}}\left|K_{n}\right| \Sigma\right\rangle & =-\left\langle\mathrm{eq}_{\mathrm{M}}\left|\hat{\Psi}_{n}^{\prime}(0)\right| \Sigma\right\rangle \\
& =\int_{0}^{\infty} \mathrm{d} t t\left\langle\mathrm{eq}_{\mathrm{M}}\left|\Psi_{n}(t)\right| \Sigma\right\rangle \\
& =\int_{0}^{\infty} \mathrm{d} t t \bar{\psi}_{n}(t) .
\end{aligned}
$$

Therefore, in this case

$$
\mu_{n}^{\mathrm{asym}}=\frac{\int_{0}^{\infty} \mathrm{d} t t \bar{\psi}_{n}(t)}{\int_{0}^{\infty} \mathrm{d} t t \bar{\psi}_{0}(t)} .
$$

This is the average of the $n$th moment weighted by its corresponding waiting time. This is explained by the fact, that the process stays with this value for the waiting time which has to be taken into account for a time average. For $\alpha<1$ this expression is not defined as the denominator is infinite.

However, also in the ergodic case the long time moments can be used to explain the next terms in the expansion (Eq. (7.33))

$$
\hat{\Psi}_{n}(\lambda)=\hat{\Psi}_{n}(0)-\lambda K_{n}+\lambda^{1+\beta} L_{n}+\mathrm{o}\left(\lambda^{1+\beta}\right)
$$

with $0<\beta<1$. Again similar to [20] (Eqs. (1.6), (1.8)), the long time behavior

$$
\int_{T}^{\infty} \mathrm{d} t \int_{-\infty}^{\infty} \mathrm{d} x x^{n} \bar{\phi}(x, t) \simeq \frac{\left\langle\mathrm{eq}_{\mathrm{M}}\left|L_{n}\right| \Sigma\right\rangle}{|\Gamma(-\beta)|} T^{-\beta-1}
$$

corresponds to the small $\lambda$ behavior of

$$
\hat{\bar{\psi}}_{n}(\lambda) \simeq \hat{\bar{\psi}}_{n}(0)-\left\langle\mathrm{eq}_{\mathrm{M}}\left|K_{n}\right| \Sigma\right\rangle \lambda+\left\langle\mathrm{eq}_{\mathrm{M}}\left|L_{n}\right| \Sigma\right\rangle \lambda^{1+\beta} .
$$

We obtain in this case

$$
\begin{aligned}
\bar{\psi}_{n}^{\mathrm{lt}} & =\lim _{T \rightarrow \infty} \frac{T^{-\beta-1}\left\langle\mathrm{eq}_{\mathrm{M}}\left|L_{n}\right| \Sigma\right\rangle /|\Gamma(-\beta)|}{T^{-\beta-1}\left\langle\mathrm{eq}_{\mathrm{M}}\left|L_{0}\right| \Sigma\right\rangle /|\Gamma(-\beta)|} \\
& =\frac{\left\langle\mathrm{eq}_{\mathrm{M}}\left|L_{n}\right| \Sigma\right\rangle}{\left\langle\mathrm{eq}_{\mathrm{M}}\left|L_{0}\right| \Sigma\right\rangle} .
\end{aligned}
$$

With this, we calculate the second moment of the long time distribution with respect to the mean value of the process

$$
\begin{aligned}
\operatorname{Var}^{\text {center }} & =\int_{-\infty}^{\infty} \mathrm{d} x\left(x-\mu_{1}^{\mathrm{asym}}\right)^{2} \bar{\phi}^{\mathrm{lt}}(x) \\
& =\bar{\psi}_{2}^{\mathrm{lt}}-2 \bar{\psi}_{1}^{\mathrm{lt}} \mu_{1}^{\mathrm{asym}}+\left(\mu_{1}^{\mathrm{asym}}\right)^{2} \\
& =\frac{\left\langle\mathrm{eq}_{\mathrm{M}}\left|L_{2}\right| \Sigma\right\rangle}{\left\langle\mathrm{eq}_{\mathrm{M}}\left|L_{0}\right| \Sigma\right\rangle}-2 \frac{\left\langle\mathrm{eq}_{\mathrm{M}}\left|L_{1}\right| \Sigma\right\rangle\left\langle\mathrm{eq}_{\mathrm{M}}\left|K_{1}\right| \Sigma\right\rangle}{\left\langle\mathrm{eq}_{\mathrm{M}}\left|L_{0}\right| \Sigma\right\rangle\left\langle\mathrm{eq}_{\mathrm{M}}\left|K_{0}\right| \Sigma\right\rangle}+\frac{\left\langle\mathrm{eq}_{\mathrm{M}}\left|K_{1}\right| \Sigma\right\rangle^{2}}{\left\langle\mathrm{eq}_{\mathrm{M}}\left|K_{0}\right| \Sigma\right\rangle^{2}}
\end{aligned}
$$


This expression appears in the low-frequency behavior of the spectrum for this case.

\section{References}

[1] Takuma Akimoto, Eli Barkai. Aging generates regular motions in weakly chaotic systems. Phys. Rev. E 87 (2013) 032915.

[2] E. Barkai. Aging in Subdiffusion Generated by a Deterministic Dynamical System. Phys. Rev. Lett. 90 (2003) 104101.

[3] E. Barkai, E. Aghion, D. Kessler. From the area under the Bessel excursion to anomalous diffusion of cold atoms. Physical Review X 4 (2014) 021036.

[4] E. Barkai, Y. C. Cheng. Aging Continuous Time Random Walks. J. of Chemical Physics 118 (2003) 6167.

[5] E. Barkai, Y. Garini, R. Metzler. Strange Kinetics of single molecules in living cells. Physics Today, 65, (2012) 29.

[6] E. Barkai, I. Sokolov. Multi-point Distribution Function for the Continuous Time Random Walk. J. of Stat. Mech: Theory and Experiment (2007) P08001.

[7] A. Baule, R. Friedrich. A fractional diffusion equation for two-point probability distributions of a continuous-time random walk. EPL 77 (2007) 10002.

[8] G. Bel, E. Barkai. Weak Ergodicity Breaking in the Continuous-Time Random Walk. Phys. Rev. Lett. 94 (2005) 240602 .

[9] G.M. Berger, B. Mandelbrot. A new model for error clustering in telephone circuits. IBM Journal of Research and Development 7 (1963) 224.

[10] S. Bianco, M. Ignaccolo, M. S. Rider, M. J. Ross, P. Winsor, P. Grigolini. Brain, music, and non-Poisson renewal processes. Phys. Rev. E 75 (2007) 061911.

[11] P. Billingsley. Probability and Measure. John Wiley \& Sons (1995).

[12] J. Bouchaud. Weak ergodicity breaking and aging in disordered systems. Journal de Physique I France 2 (1992) 17051713.

[13] J. P. Bouchaud, A. Georges. Anomalous diffusion in disordered media: Statistical mechanisms, models and physical applications. Phys. Rep., 195, (1990) 127.

[14] S. Burov, R. Metzler, E. Barkai. Ageing and non-ergodicity beyond the Khinchin theorem. Proceedings of the National Academy of Sciences, 107, (2010) 13228.

[15] A. Dechant, E. Lutz. Wiener-Khinchin Theorem for Non stationary Scale-Invariant Processes. Phys. Rev. Lett., 115 (2015) 080603.

[16] J. L. Doob. Renewal theory from the point of view of the theory of probability. Transactions of the American Mathematical Society, Vol. 63, (1948), 422-438.

[17] E. B. Dynkin. Some limit theorems for sums of independent random variables with infinite mathematical expectations. Selected Translations Math. Stat. Prob 1 (1961) 417; ibid. Izv. Akad. Nauk SSSR Ser. Mat. 19 (1955) 247.

[18] S. Ferraro, M. Manzini, A. Masoero, E. Scalas. A random telegraph signal of Mittag-Leffler type. Physica (Amsterdam) 388A (2009) 3991.

[19] H.C. Fogedby. Langevin equations for continuous time Lévy flights. Phys. Rev. E, 50 (1994) 1657.

[20] C. Godrèche, J. M. Luck. Statistics of the occupation time of renewal processes. J. Stat. Phys. 104 (2001) 489-524.

[21] C. Godrèche, S. N. Majumdar, G. Schehr. Statistics of the longest interval in renewal processes. J. Stat. Mech. (2015) $\mathrm{P} 03014$.

[22] J.-H. Jeon, E. Barkai, R. Metzler. Noisy continuous time random walks. J. Chemical Physics 139 (20130 121916.

[23] Y. Jung, E. Barkai, R. J.Silbey. Lineshape theory and photon counting statistics for blinking quantum dots: a Lévy walk process. Chemical Physics 284 (2002) 181-194.

[24] Tosio Kato. Perturbation Theory for Linear Operators. Volume 132 of Grundlehren der mathematischen Wissenschaften. Springer, Berlin, 1980.

[25] J. Klafter, M. F. Shlesinger, G. Zumofen. Beyond Brownian motion. Physics Today, 49 (1996) 33.

[26] N. Leibovich, E. Barkai. Aging Wiener-Khinchin Theorem. Phys. Rev. Lett., 115 (2015) 080602.

[27] S. B. Lowen, M. C. Teich. Fractal renewal processes generate 1/f noise. Phys. Rev. E, 47(1993) $992-1001$.

[28] F. Mainardi, R. Gorenflo, A. Vivoli. Renewal processes of Mittag Leffler and Wright Type. Fractional Calculus and Applied Analysis, 8 (2005) 7-38.

[29] B. Mandelbrot. Some noises with $1 / f$ spectrum, a bridge between direct current and white noise. IEEE Trans. Inform. Theory, IT-13 (1967) 289-298.

[30] C. Manzo, J. A. Torreno-Pina, P. Massignan, G. J. Lapeyre, Jr., M. Lewenstein, M. F. Garcia Parajo. Weak Ergodicity Breaking of Receptor Motion in Living Cells Stemming from Random Diffusivity. Phys. Rev. X 5 (2015) 011021.

[31] G. Margolin, E. Barkai. Non-ergodicity of a Time Series Obeying Lévy Statistics. J. Stat. Phys., 122 (2006) 137.

[32] M. M. Meerschaert, H. P. Scheffler. Limit theorems for continuous time random walks with infinite waiting times. J. Appl. Prob., 41 (2004) 623-638.

[33] M. M. Meerschaert, P. Straka. Inverse stable subordinators. Mathematical Modelling of Natural Phenomena, 8 (2013) $1-16$.

[34] M. M. Meerschaert, P. Straka. Semi-Markov approach to continuous time random walk limit processes. Annals of Probability, 42 (2014) 1699-1723.

[35] R. Metzler, J. H.Jeon, A. G. Cherstvy, E. Barkai. Anomalous diffusion models and their properties: non-stationarity, non-ergodicity, and ageing at the centenary of single particle tracking. Phys. Chem. Chem. Phys., 16 (2014) 24128. 
[36] R. Metzler, J. Klafter. The random walk's guide to anomalous diffusion: a fractional dynamics Phys. Rep., 339 (2000) 1.

[37] A. Moinet, M. Starnini, R. Pastor-Satorras. Burstiness and Aging in Social Temporal Networks. Phys. Rev. Lett., 114 (2015) 1108701.

[38] C. Monthus, J-P. Bouchaud. Models of traps and glass phenomenology J. Phys. A: Math. Gen., 29 (1996) $3847-3869$.

[39] M. Niemann, H. Kantz. Joint probability distribution and multipoint correlations of the continuous time random walk. Phys. Rev. E., 28 (2008) 051104.

[40] M. Niemann, H. Kantz, E. Barkai. Fluctuations of $1 / f$ Noise and the Low-Frequency Cutoff Paradox. Phys. Rev. Lett., 110 (2013) 140603.

[41] A. V. Oppenheim, R. W. Schafer. Discrete-Time Signal Processing. Pearson Education Limited (2013).

[42] M. Politi, T. Kaizoji, E. Scalas. Full Characterization of the fractional Poisson process Europhys. Lett., 96 (2011) 20004.

[43] M. A. Rodriguez. Class of perfect $1 / f$ noise and the low frequency cutoff paradox. Phys. Rev. E, 92 (2015) 012112.

[44] S. Sadegh, E. Barkai, D. Krapf. 1/f noise for intermittent quantum dots exhibits non-stationarity and critical exponents New. J. of Physics, 16 (2014) 113054.

[45] F. Sanda, S. Mukamel. Anomalous line-shapes and aging effects in two-dimensional correlation spectroscopy. J. Chem. Physics, 127, 154107 (2007).

[46] J. H. P. Schulz, E. Barkai, R. Metzler. Aging effects and population splitting in single-particle trajectory averages. Phys. Rev. Lett., 110 (2013) 020602.

[47] F. D. Stefani, J. P. Hoogenboom, E. Barkai. Beyond quantum jumps: Blinking nano-scale light emitters. Physics Today, 62 (2009) 34.

[48] K. A. Takeuchi, T. Akimoto. Characteristic Sign Renewals of Kardar-Parisi-Zhang Fluctuations. [cond-mat.stat-mech] (2015) arXiv:1509.03082.

[49] S. Vajna, B. Tóth, J. Kertész. Modelling bursty time series. New J. Phys., 15 (2013) 103023.

[50] A.V. Weigel, B. Simon, M.M. Tamkun, D. Krapf. Ergodic and nonergodic processes coexist in the plasma membrane as observed by single-molecule tracking. Proceedings of the National Academy of Sciences, 108 (2011) 6438.

[51] I.Y. Wong, M.L. Gardel, D.R. Reichmann, E.R. Weeks, M.T. Valentine, A.R. Bausch, D.A. Weitz. Anomalous Diffusion Probes Microstructure Dynamics of Entangled F-Actin Networks. Phys. Rev. Lett., 92, (2004) 1781011. 